\title{
A review on the thermal hydraulic characteristics of the air-cooled heat exchangers in forced convection
}

\author{
ANKUR KUMAR ${ }^{1,2}$, JYESHTHARAJ B JOSHI ${ }^{1,3, *}$, \\ ARUN K NAYAK $^{1,2}$ and PALLIPPATTU K VIJAYAN ${ }^{1,2}$ \\ ${ }^{1}$ Homi Bhabha National Institute, Anushaktinagar, Mumbai 400 094, India \\ ${ }^{2}$ Reactor Engineering Division, Bhabha Atomic Research Center, \\ Trombay, Mumbai 400 085, India \\ ${ }^{3}$ Department of Chemical Engineering, Institute of Chemical Technology, \\ Matunga, Mumbai 400 019, India \\ e-mail: jbjoshi@gmail.com
}

MS received 23 December 2014; accepted 9 January 2015

\begin{abstract}
In this paper, a review is presented on the experimental investigations and the numerical simulations performed to analyze the thermal-hydraulic performance of the air-cooled heat exchangers. The air-cooled heat exchangers mostly consist of the finned-tube bundles. The primary role of the extended surfaces (fins) is to provide more heat transfer area to enhance the rate of heat transfer on the air side. The secondary role of the fins is to generate vortices, which help in enhancing the mixing and the heat transfer coefficient. In this study, the annular and plate fins are considered, the annular fins are further divided into four categories: (1) plane annular fins, (2) serrated fins, (3) crimped spiral fins, (4) perforated fins, and similarly for the plate fins, the fin types are: (1) plain plate fins, (2) wavy plate fins, (3) plate fins with DWP, and (4) slit and strip fins. In Section 4, the performance of the various types of fins is presented with respect to the parameters: (1) Reynolds number, (2) fin pitch, (3) fin height, (4) fin thickness, (5) tube diameter, (6) tube pitch, (7) tube type, (8) number of tube rows, and (9) effect of dehumidifying conditions. In Section 5, the conclusions and the recommendations for the future work have been given.
\end{abstract}

Keywords. Air cooled heat exchanger, forced convection, finned-tubes, air flow.

\section{Introduction}

The air cooling technology is used in a variety of applications, for example automobile industry, power plants, computer systems, and air conditioners. In the power plants, the air cooling is used in the air cooled condensers, and dry and wet cooling towers. The A-type air-cooled condensers (figure 1) are used to condense the exhaust steam from the turbine, whereas the dry and wet

*For correspondence 


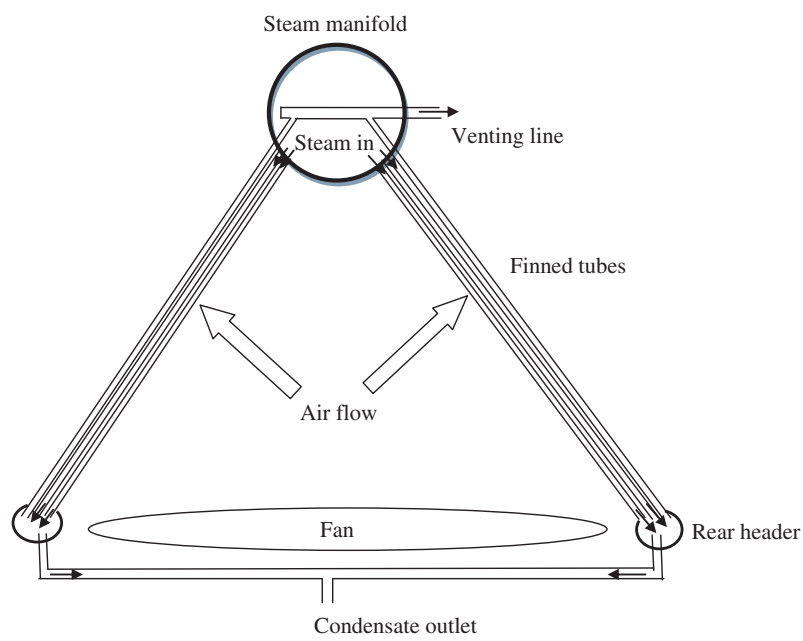

Figure 1. Schematic representation of A type air cooled condenser.

cooling towers are used to remove the heat from the secondary water loop by forced or natural draft of air. For the latter method, approximately $2.11 \mathrm{~L}$ of water is needed per $\mathrm{kWh}$ of energy produced. Due to heavy demand of water in cooling towers, substantial amount of research work has been published in the past 50 years. The performance of the air-cooled heat exchangers depends upon many geometrical parameters, like fin type, fin spacing, number of tube rows, and tube pitch. The ambient parameters like wind and humidity are also important in determining the efficiency of the air-cooled heat exchangers. One of the major problems with the air cooling technology is the low heat transfer coefficient provided by air, which results in a large heat transfer area of the heat exchanger and therefore, a high associated capital cost as compared to the watercooled heat exchanger. The total cost associated with the A-type air-cooled condensers includes the capital cost, operating cost, and the cost of the space used, and these three parameters must be optimized to obtain an economical air cooled condenser. Continuous efforts have been going on to improve the performance and the efficiency of the air-cooled heat exchanger, still, there is a lot of scope to make the air cooling technology more economical and efficient.

A large number of researchers have studied the effect of the geometric parameters on the heat transfer and pressure drop characteristics of the air-cooled heat exchangers. The experimental as well as the numerical simulations have been performed to investigate the effects of various parameters. Ota et al (1984) and Badr (1994) have reviewed the studies performed on the heat transfer and flow field around the bare elliptical tubes. Theoretical studies on the natural and the forced convection over elliptical tubes have been performed by Chao \& Fagbenle (1974), and Merkin (1977). In early 1990s, Kayansayan (1993) experimentally studied the effects of fin spacing, number of rows, and number of tubes per row on the heat transfer and the pressure drop characteristics of the plate- finned-tube heat exchangers. After that many researchers, Wang and coworkers (1997, 2000, 2000a), Jang \& Yang (1998), Ay and group (2002), Nuntaphan et al (2005a, b), He and group (2005), etc., have performed studies on the effect of geometric parameters on the heat transfer and the pressure drop characteristics of the air-cooled heat exchanger. Many correlations have been proposed for the air-side heat transfer coefficient for various types of fins. It is well known that the temperature distribution on the fin surface is not uniform, and the heat transfer coefficient varies over the fin surface. Chen and group (2005, 2007; 
Chen \& Hsu 2008; Chen \& Lai 2012) conducted various studies to determine the heat transfer coefficient variation on the fin surface. Previous work on the air-cooled heat exchangers shows that the thermal-hydraulic behavior of various types of fins differs from each other; therefore, depending on the application, the design of the finned tube has to be optimized to obtain a maximum heat transfer rate per unit power consumption. The thermal and mechanical properties of the materials play an important role in determining the thermal-hydraulic performance of heat exchangers. Aluminum, copper, steel, and nickel alloys are mostly used as fin and tube materials. Out of these, copper has the highest thermal conductivity, and hence provides a better fin efficiency than the other materials. Aluminum has the second best thermal conductivity, and hence, provides the second best fin efficiency (slightly lower than $\mathrm{Cu}$ ). However, copper finned-tubes are almost twice as costly as aluminum finned-tubes, therefore, this factor must be taken into account along with the fin efficiency while designing an economical and efficient air-cooled heat exchanger.

In this paper, a critical analysis of the previous published work has been presented. The system details for the experiments and the numerical simulations of the previous studies are given in table 1 and table 2, respectively. The correlations proposed by various authors for the heat transfer and the pressure loss are provided in table 3 and table 4, respectively.

\section{Important parameters}

In this section, role of three important parameters (Reynolds number, area goodness factor and the volume goodness factor) has been discussed. The first parameter is the Reynolds number, which is a dimensionless number and is defined as the ratio of the inertial forces to the viscous forces. The Reynolds number is used to predict the different flow regimes, such as creepy, laminar and turbulent in fluid flows. The Reynolds number is defined as

$$
R e=\frac{\rho U_{\max } l}{\mu}
$$

where $l$ is the characteristic length. In literature, the definition of the characteristic length has been different for different authors. One category of the authors considers the outer tube diameter or the tube collar diameter as the characteristic length, the second category of the authors considers the fin spacing as the characteristics length, and in the third category, the hydraulic diameter of the heat exchanger is considered as the characteristic length. However, in most of the cases, the outer tube diameter is considered as the characteristic length, therefore, in the present review, we have used the outer tube diameter (or tube collar diameter) as the length dimension in the Reynolds number and wherever it was possible, we have converted the Reynolds number based on other characteristics length to the Reynolds number based on the outer tube diameter and the results are given in table 1 and table 2 . However, in some studies we have retained the Reynolds number as it was used by the authors, and in tables 1 and 2 they have been provided with a separate subscript.

The performance evaluation criterions (PECs) for any heat exchanger comprise mainly two parameters, area goodness factor and the volume goodness factor (Sahiti et al 2006). When the frontal area of the heat exchanger is the parameter of interest, then the area goodness factor is compared for the heat exchangers. The area goodness factor can be represented by the comparison of $j / f$ ratio with respect to the Reynolds number. A high value of $j / f$ ratio with respect to the Reynolds number indicates that a less frontal area is required of the heat exchanger for 
Table 1. Summary of published work (experimental).

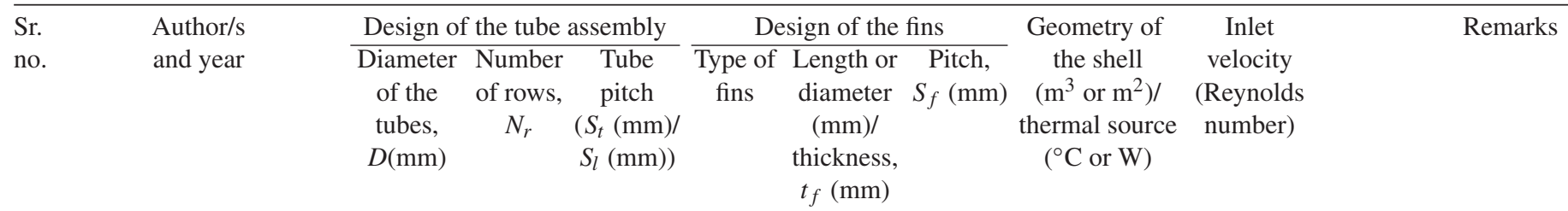

1. Saboya \& Sparrow (1976)

8.53

$21.3 /$

Plate $\begin{gathered}37 \mathrm{~mm} \\ \text { (along the } \\ \text { flow)/ }\end{gathered}$

2. Fiebig et al (1993)

32

3

$64 / 47.25$

$\begin{array}{cc}\begin{array}{c}\text { Plate } \\ \text { with }\end{array} & \begin{array}{c}\text { (along the } \\ \text { DWP }\end{array} \\ \text { flow) } / 0.84\end{array}$

7.84

$160 \times$ 320/Electrical heating

(211-1089, The mass transfer coefficient $\left.R e_{h}\right)$ was affected by the boundary layer development for the first row, while the vortex generation gave more contribution to the mass transfer coefficient for the second row.

3. Kayansayan (1993)

$9.52,12.5, \quad 4$

25.4-40/ Plate
22-34.67

$0.2 \mathrm{~mm}$

0.0022

$50 \times 50 / \mathrm{Hot}$
water $(80)$

to
0.0042

4. $\quad$ Mirth \&

$13.2-16.4 \quad 4-8$

Wavy

$/ 0.15$

$1.62-$

Ramadhyani (1993)

3.20

\section{/Cold water}

(3-6.3)

5. Hu \& Jacobi (1993)
$40 \times 40 \times$ 135

1.45-6.3 The increment in the heat transfer (2700 - and pressure drop for the inline 12340) arrangement with the DWP was $55-60 \%$ and $20-45 \%$, respectively.

0-15 The Colburn factor (j) decreases (100- with an increase in the finning 30000) factor and the Reynolds number.

1-2.9 The Nusselt number correlation (1350 - for the dry surface predicted the 4570) heat transfer for wet surface within $\pm 5 \%$.

13711- The heat and mass transfer 49858 analogy was applied to obtain the fin efficiency as a function of the fin parameter (fin diameter, thermal conductivity and heat transfer coefficient). 
Table 1. (contd)

\begin{tabular}{|c|c|c|c|c|c|c|c|c|c|c|}
\hline \multirow{2}{*}{$\begin{array}{l}\text { Sr. } \\
\text { no. }\end{array}$} & \multirow{2}{*}{$\begin{array}{l}\text { Author/s } \\
\text { and year }\end{array}$} & \multicolumn{3}{|c|}{ Design of the tube assembly } & \multicolumn{3}{|c|}{ Design of the fins } & \multirow[b]{2}{*}{$\begin{array}{l}\text { Geometry of } \\
\text { the shell } \\
\left(\mathrm{m}^{3} \text { or } \mathrm{m}^{2}\right) / \\
\text { thermal source } \\
\left({ }^{\circ} \mathrm{C} \text { or } \mathrm{W}\right)\end{array}$} & \multirow{2}{*}{$\begin{array}{l}\text { Inlet } \\
\text { velocity } \\
\text { (Reynolds } \\
\text { number) }\end{array}$} & \multirow[t]{2}{*}{ Remarks } \\
\hline & & $\begin{array}{l}\text { Diameter } \\
\text { of the } \\
\text { tubes, } \\
D(\mathrm{~mm})\end{array}$ & $\begin{array}{l}\text { Number } \\
\text { of rows, } \\
\quad N_{r}\end{array}$ & $\begin{array}{c}\text { Tube } \\
\text { pitch } \\
\left(S_{t}(\mathrm{~mm}) /\right. \\
\left.S_{l}(\mathrm{~mm})\right)\end{array}$ & $\begin{array}{l}\text { Type of } \\
\text { fins }\end{array}$ & $\begin{array}{l}\text { Length or } \\
\text { diameter } \\
(\mathrm{mm}) / \\
\text { thickness, } \\
t_{f}(\mathrm{~mm})\end{array}$ & $\begin{array}{c}\text { Pitch, } \\
S_{f}(\mathrm{~mm})\end{array}$ & & & \\
\hline 6. & $\begin{array}{c}\text { Mirth \& } \\
\text { Ramadhyani } \\
\text { (1994) }\end{array}$ & $13.2-16.4$ & $4-8$ & & Wavy & $/ 0.15$ & $\begin{array}{c}1.47- \\
3.05\end{array}$ & $\begin{array}{l}\text { /Cold water } \\
(3-6.3)\end{array}$ & $\begin{array}{c}1-2.9 \\
(1350- \\
4570)\end{array}$ & $\begin{array}{l}\text { The length of the coil and the } \\
\text { fin spacing affected the Nusselt } \\
\text { number for all the coils. However, } \\
\text { the friction factor for one set of } \\
\text { coil was a function of the fin } \\
\text { spacing only, and for the other } \\
\text { set, it was a function of both the } \\
\text { fin spacing and length of the coil. }\end{array}$ \\
\hline 7. & $\begin{array}{c}\text { Fiebig et al } \\
\quad(1994)\end{array}$ & $\begin{array}{l}32 \text { (round } \\
\text { tubes) } \\
69.6 \times 12 \\
\text { (elliptical) }\end{array}$ & 3 & $64 / 47.25$ & $\begin{array}{l}\text { Plate } \\
\text { with } \\
\text { DWP }\end{array}$ & $\begin{array}{l}216 \mathrm{~mm} \\
\text { (along the } \\
\text { flow)/0.84 }\end{array}$ & 7.84 & $\begin{array}{l}160 \times \\
320 / \text { Electrical } \\
\text { heating }\end{array}$ & $\begin{array}{l}1.45-6.3 \\
(2700- \\
12340)\end{array}$ & $\begin{array}{l}\text { The DWP affected the flat tubes } \\
\text { to a larger extent as compared to } \\
\text { the round tubes. The increment in } \\
\text { the heat transfer for the flat tubes } \\
\text { was } 80-120 \% \text { and for round tubes } \\
\text { it was } 10 \% \text {. }\end{array}$ \\
\hline 8. & $\begin{array}{l}\text { Tiggelbeck } \\
\text { et al (1994) }\end{array}$ & & & & $\begin{array}{l}\text { Plate } \\
\text { with } \\
\text { VGs }\end{array}$ & & & /Hot air, 50 & $\begin{array}{c}(2000- \\
9000 \\
\left.R e_{h}\right)\end{array}$ & $\begin{array}{l}\text { The performance of the DWP and } \\
\text { the RWP was studied at various } \\
\text { attack angles. It was found that, } \\
\text { the maxima in the Nusselt number } \\
\text { occurs between } 50^{\circ} \text { and } 70^{\circ} \text { for } \\
\text { DWP and between } 45^{\circ} \text { and } 65^{\circ} \\
\text { for RWP. }\end{array}$ \\
\hline 9. & $\begin{array}{c}\text { Wang } \\
\text { et al }(1996)\end{array}$ & 9.52 & $2-6$ & $25.4 / 22$ & Plate & $/ 0.13-2$ & $\begin{array}{c}1.74- \\
3.20\end{array}$ & $\begin{array}{l}\text { /Hot water } \\
\quad(60)\end{array}$ & $280-6980$ & $\begin{array}{l}\text { The Colburn factor was found to } \\
\text { be independent of the number of } \\
\text { tube rows and slightly dependent } \\
\text { on the fin thickness and fin spacing. }\end{array}$ \\
\hline
\end{tabular}


Table 1. (contd)

\begin{tabular}{|c|c|c|c|c|c|c|c|c|c|c|}
\hline $\begin{array}{l}\text { Sr. } \\
\text { no. }\end{array}$ & $\begin{array}{l}\text { Author/s } \\
\text { and year }\end{array}$ & \multicolumn{3}{|c|}{ Design of the tube assembly } & \multicolumn{3}{|c|}{ Design of the fins } & $\begin{array}{l}\text { Geometry of } \\
\text { the shell } \\
\left(\mathrm{m}^{3} \text { or } \mathrm{m}^{2}\right) / \\
\text { thermal source } \\
\left({ }^{\circ} \mathrm{C} \text { or } \mathrm{W}\right)\end{array}$ & $\begin{array}{l}\text { Inlet } \\
\text { velocity } \\
\text { (Reynolds } \\
\text { number) }\end{array}$ & Remarks \\
\hline 10 & Wang et al (1997) & 9.53 & $1-4$ & $\begin{array}{l}25.4 \\
29.4 / 19.05 \\
29.4\end{array}$ & Wavy & $/ 0.12,0.2$ & $\begin{array}{c}1.69 \text { to } \\
3.53\end{array}$ & $\begin{array}{l}\text { /Hot water } \\
\text { (60) }\end{array}$ & $\begin{array}{l}0.3-5.5 \\
(372- \\
7456)\end{array}$ & $\begin{array}{l}\text { The heat transfer increased with an } \\
\text { increase in the number of rows for } \\
R e<900 \text {, and for } R e>900 \text {, the } \\
\text { heat transfer decreased with an } \\
\text { increase in the number of rows for } \\
\text { the staggered arrangement and did } \\
\text { not show any variation for the } \\
\text { inline arrangement. The friction } \\
\text { factor was almost independent of } \\
\text { the number of rows. }\end{array}$ \\
\hline 12 & Madi et al (1998) & 9.956 & $1-4$ & $\begin{array}{c}19- \\
25.4 / \\
16-22\end{array}$ & $\begin{array}{l}\text { Plain and } \\
\text { wavy }\end{array}$ & /0.12-0.2 & $\begin{array}{c}1.615- \\
4.129\end{array}$ & $\begin{array}{c}\text { /Hot water, } \\
84\end{array}$ & $\begin{array}{l}1-20 \\
(220- \\
6700 \\
\left.R e_{h}\right)\end{array}$ & $\begin{array}{l}\text { The wavy fin was found to have a larger } \\
\text { Colburn factor and friction factor as } \\
\text { compared to the plain fins. }\end{array}$ \\
\hline 13 & Yun \& Lee (1999) & $22.5 / 7.5$ & 2 & $\begin{array}{l}63 \\
21 / 38.1 \\
12.7\end{array}$ & $\begin{array}{l}\text { Plate } \\
\text { with slits } \\
\text { and } \\
\text { louver }\end{array}$ & $/ 0.3,0.1$ & $3.6 / 1.2$ & $\begin{array}{c}\text { /Hot water at } \\
45\end{array}$ & $\begin{array}{l}0.2-1.5 \\
(300- \\
2230 \\
\left.R e_{h}\right)\end{array}$ & $\begin{array}{l}\text { Different slit patterns had a negligible } \\
\text { effect on the heat transfer coefficient } \\
\text { but the effect on the pressure drop was } \\
\text { quite significant. } \\
\text { The strips in the rear part of the plain } \\
\text { fin were recommended. }\end{array}$ \\
\hline
\end{tabular}


Table 1. (contd)

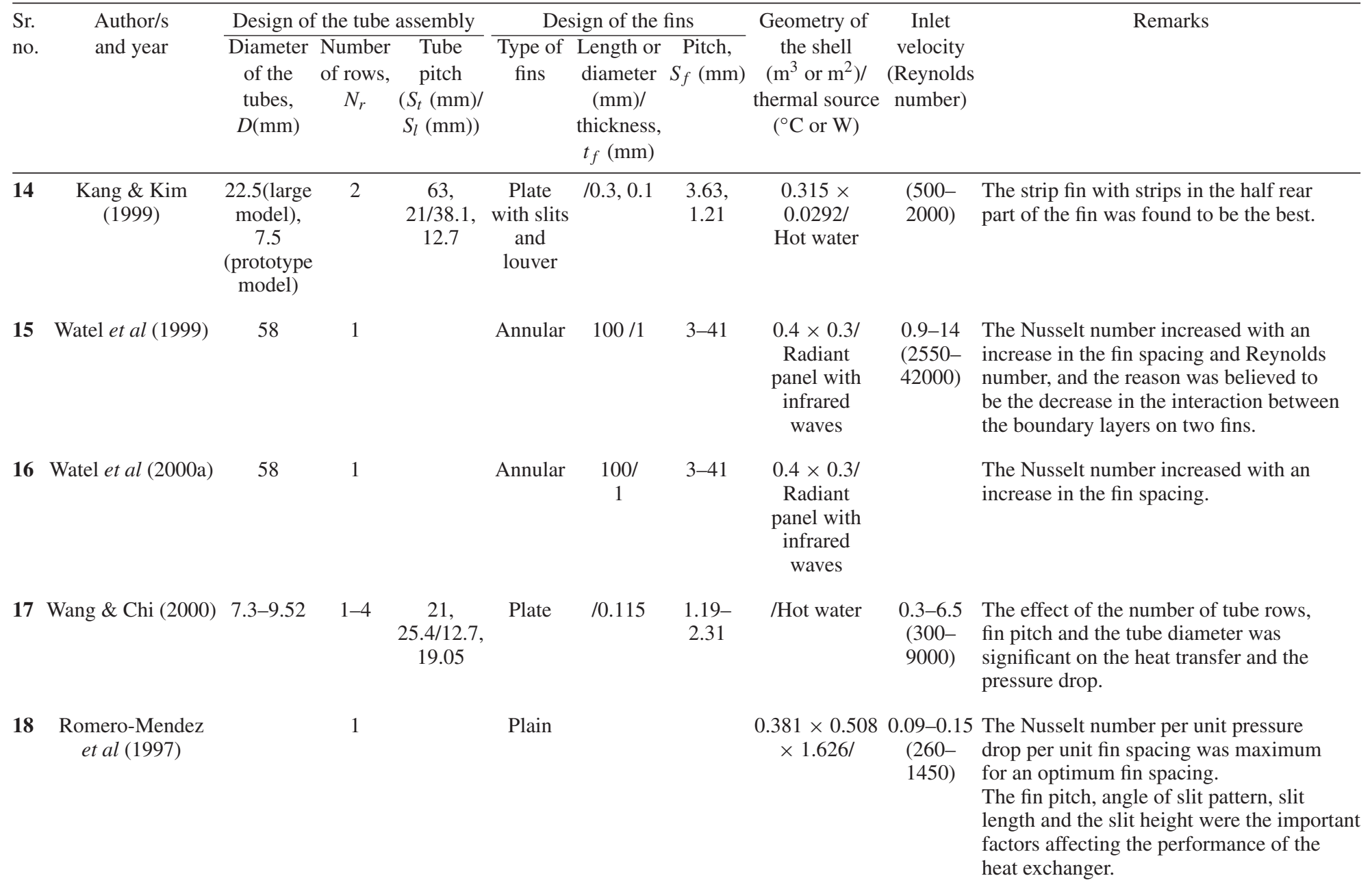


Table 1. (contd)

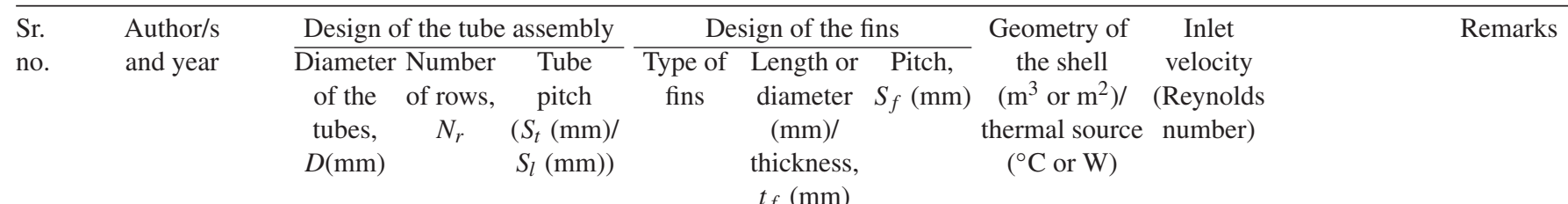

\begin{tabular}{llllclll}
\hline 19 & Du \& Wang (2000) & $7.3-14.8$ & $1-6$ & $\begin{array}{c}17.32- \\
38 / 15-33\end{array}$ & Slit & $/ 0.11-0.18$ & $1.20-$ \\
& & & & & 2.50
\end{tabular}

20

Yun \& Lee (2000)

$22.5 / 7.5$

2

63,21/38. Plate

$1,12.7$ with slits

/0.3, $0.1 \quad 3.6 / 1.2$

ton

21 Yan \& Sheen (2000)

9.53

$1-4$

$25.4 /$
19.05

Plate,

wavy

and

louver

22 Watel et al (2000b)

58

1

Annular

$100 /$

3-41

$0.4 \times 0.3 /$
Hot water

23 Wang et al (2000b)

8.62
(65)

(200- The effect of fin pitch on the heat transfer

8000) performance variedfor two types of fins, and the difference was found to be because of the difference in the manufacturing design of the fins.

/Electric

heating

0.2-1.5 It was found that the effect of four factors (300- fin pitch (39\%), angle of slit pattern

2230, (28\%), slit length (20\%), and slit height $\left.R e_{h}\right) \quad(9 \%)$ was significant on the performance of the heat exchanger.

$0.6 \times 0.4 / \quad 0.47-3.19$ The heat transfer per unit pumping

Hot water $\quad(650-$ power, the volume goodness factor, and

(60) 3500) surface area reduction for a fixed power was maximum for the louver fin.

0.9-6 The Nusselt number was found to be (2550 - largely dependent on the air flow Re 18200) number as compared to the rotational Re number.

0.3-3.5 The effect of the waffle height on the

(150- heat transfer was negligible, but the

1800) effect on the pressure drop was significant. The heat transfer was found to be a strong function of the fin pitch for the larger waffle height. $\begin{array}{cc}1.70- & 0.85 \times 0.55 / \\ 3.14 & \text { Cold water }\end{array}$ 
Table 1. (contd)

\begin{tabular}{|c|c|c|c|c|c|c|c|c|c|c|}
\hline $\begin{array}{l}\text { Sr. } \\
\text { no. }\end{array}$ & $\begin{array}{l}\text { Author/s } \\
\text { and year }\end{array}$ & \multicolumn{3}{|c|}{ Design of the tube assembly } & \multicolumn{3}{|c|}{ Design of the fins } & $\begin{array}{c}\text { Geometry of } \\
\text { the shell } \\
\left(\mathrm{m}^{3} \text { or } \mathrm{m}^{2}\right) / \\
\text { thermal source } \\
\left({ }^{\circ} \mathrm{C} \text { or } \mathrm{W}\right)\end{array}$ & $\begin{array}{c}\text { Inlet } \\
\text { velocity } \\
\text { (Reynolds } \\
\text { number) }\end{array}$ & Remarks \\
\hline 24 & $\begin{array}{c}\text { Saboya \& } \\
\text { Saboya (2001) }\end{array}$ & $\begin{array}{l}12.06 \times \\
6.03-17.06 \\
\times 8.53 \\
\text { (elliptical) }\end{array}$ & $1-2$ & $\begin{array}{c}21.35 / \\
18.5\end{array}$ & Plate & & 1.70 & & $150-1300$ & $\begin{array}{l}\text { The elliptical tubes did not provide } \\
\text { adverse change in the average Sherwood } \\
\text { number, however, elliptical tubes } \\
\text { provided a higher fin efficiency. }\end{array}$ \\
\hline 25 & Torii et al (2002) & 30 & 3 & $75 / 75$ & $\begin{array}{l}\text { Platewith } \\
\text { DWP }\end{array}$ & & 5.6 & $\begin{array}{c}0.15 \times 0.1 \times \\
0.3 / \\
\text { Electrical } \\
\text { heating }\end{array}$ & $\begin{array}{c}0.5-3.5 \\
(937- \\
\left.6428, \operatorname{Re}_{f r}\right)\end{array}$ & $\begin{array}{l}\text { The common flow up configuration of the } \\
\text { DWP enhanced the heat transfer and } \\
\text { reduced the pressure drop as compared to } \\
\text { the common flow down configuration. }\end{array}$ \\
\hline 26 & Kwak et al (2002) & 30 & $1-3$ & $75 / 75$ & $\begin{array}{l}\text { Plate } \\
\text { with } \\
\text { DWP }\end{array}$ & & 5.6 & $\begin{array}{c}0.15 \times 0.1 \times \\
0.3 / \\
\text { Electrical } \\
\text { heating }\end{array}$ & $\begin{array}{c}0.5-3.5 \\
(937- \\
\left.6428, \operatorname{Re}_{f r}\right)\end{array}$ & $\begin{array}{l}\text { An increment of } 10-25 \% \text { in the heat transfer } \\
\text { and } 20-30 \% \text { in the pressure drop was } \\
\text { observed with DWP included for the inline } \\
\text { arrangement. }\end{array}$ \\
\hline 27 & $\begin{array}{l}\text { ElSherbini \& } \\
\text { Jacobi (2002) }\end{array}$ & 9.5 & 8 & & $\begin{array}{l}\text { Plate } \\
\text { with } \\
\text { DWP }\end{array}$ & & 5 & $\begin{array}{l}0.058 \times 0.61 / \\
\text { Electrical } \\
\text { heating }\end{array}$ & $\begin{array}{l}(700- \\
2300 \\
\left.R e_{h}\right)\end{array}$ & $\begin{array}{l}\text { An overall enhancement of } 29-33 \% \text { in the } \\
\text { heat transfer was observed for the larger } \\
\text { DWP. The smaller delta winglet enhanced } \\
\text { the heat transfer by } 17-20 \% \text {. }\end{array}$ \\
\hline 28 & Ay et al (2002) & 25.4 & 3 & $\begin{array}{l}60.7 / 52.6 \\
60.7\end{array}$ & Plate & $\begin{array}{l}196 \times 240 / \\
0.5\end{array}$ & $\begin{array}{c}10.5- \\
20.5\end{array}$ & $\begin{array}{l}\text { /Electrical } \\
\text { heating }\end{array}$ & $\begin{array}{l}0.5-7.5 \\
(730- \\
11000)\end{array}$ & $\begin{array}{l}\text { The heat transfer coefficient was found to be } \\
14-32 \% \text { larger for the staggered arrangement } \\
\text { as compared to the inline arrangement. }\end{array}$ \\
\hline 29 & $\begin{array}{l}\text { Hashizume } \\
\text { et al (2002) }\end{array}$ & 31.8 & 5 & & Serrated & $68.5 /$ & 5.1 & $\begin{array}{l}\text { /Electrical } \\
\text { heating }\end{array}$ & $\begin{array}{l}(5000- \\
50000)\end{array}$ & $\begin{array}{l}\text { The correlation for the fin efficiency was } \\
\text { proposed. }\end{array}$ \\
\hline
\end{tabular}


Table 1. (contd)

\begin{tabular}{|c|c|c|c|c|c|c|c|c|c|c|}
\hline $\begin{array}{l}\text { Sr. } \\
\text { no. }\end{array}$ & $\begin{array}{l}\text { Author/s } \\
\text { and year }\end{array}$ & \multicolumn{3}{|c|}{ Design of the tube assembly } & \multicolumn{3}{|c|}{ Design of the fins } & $\begin{array}{l}\text { Geometry of } \\
\text { the shell } \\
\left(\mathrm{m}^{3} \text { or } \mathrm{m}^{2}\right) / \\
\text { thermal source } \\
\left({ }^{\circ} \mathrm{C} \text { or } \mathrm{W}\right)\end{array}$ & $\begin{array}{l}\text { Inlet } \\
\text { velocity } \\
\text { (Reynolds } \\
\text { number) }\end{array}$ & Remarks \\
\hline 30 & Kwak et al (2003) & 30 & $2-5$ & $75 / 75$ & $\begin{array}{l}\text { Plate } \\
\text { with } \\
\text { DWP }\end{array}$ & & 5.6 & $\begin{array}{c}0.15 \times 0.1 \times \\
0.3 / \\
\text { Electrical } \\
\text { heating }\end{array}$ & $\begin{array}{c}0.5-3.5 \\
(937- \\
\left.6428, R e_{f r}\right)\end{array}$ & $\begin{array}{l}\text { The three rows of finned tube with DWP } \\
\text { performed better than the } 2,4 \text {, and } 5 \text { rows. } \\
\text { The increment in the heat transfer was } \\
30-10 \% \text { with a reduction of } 55-34 \% \text { in the } \\
\text { pressure drop. }\end{array}$ \\
\hline 31 & $\begin{array}{l}\text { Wongwises \& } \\
\text { Chokeman } \\
\text { (2004) }\end{array}$ & 9.53 & $2-6$ & $\begin{array}{c}25.4 / 19.0 \\
5\end{array}$ & Wavy & $\begin{array}{c}/ 0.115- \\
0.250\end{array}$ & $\begin{array}{l}1.41- \\
2.54\end{array}$ & $\begin{array}{l}0.43 \times 0.48 / \\
\text { Hot water } \\
(55-65)\end{array}$ & $\begin{array}{c}1-6(900- \\
5400)\end{array}$ & $\begin{array}{l}\text { The Colburn factor and the friction factor } \\
\text { both increased with an increase in the fin } \\
\text { thickness for } 2 \text { row coil. For } 4 \text { and } 6 \text { row } \\
\text { coil, the Colburn factor decreased with an } \\
\text { increase in the fin thickness at low } R e \text {, and } \\
\text { at higher } R e \text {, the effectwas same as was for } 2 \\
\text { row coil. }\end{array}$ \\
\hline 33 & $\begin{array}{l}\text { Matos et al } \\
\quad(2004 \mathrm{a})\end{array}$ & $\begin{array}{l}15.875- \\
28.58 \\
\text { (circular) } \\
\text { lliptical }\end{array}$ & 4 & & Plate & $\begin{array}{c}150 \times 130 / \\
0.3\end{array}$ & & $\begin{array}{c}0.161 \times 0.152 / \\
\text { Electrical } \\
\text { heating }\end{array}$ & $\begin{array}{l}0.1-1 \\
(100- \\
1000)\end{array}$ & $\begin{array}{l}\text { The optimum value of the parameters } \\
\text { [dimensionless tube spacing }(0.5) \text {, } \\
\text { eccentricity }(0.5) \text { and dimensionless fin } \\
\text { spacing }(0.006) \text { ] were obtained. }\end{array}$ \\
\hline
\end{tabular}


Table 1. (contd)

\begin{tabular}{|c|c|c|c|c|c|c|c|c|c|c|}
\hline $\begin{array}{l}\text { Sr. } \\
\text { no. }\end{array}$ & $\begin{array}{l}\text { Author/s } \\
\text { and year }\end{array}$ & \multicolumn{3}{|c|}{ Design of the tube assembly } & \multicolumn{3}{|c|}{ Design of the fins } & $\begin{array}{l}\text { Geometry of } \\
\text { the shell } \\
\left(\mathrm{m}^{3} \text { or } \mathrm{m}^{2}\right) / \\
\text { thermal source } \\
\left({ }^{\circ} \mathrm{C} \text { or } \mathrm{W}\right)\end{array}$ & $\begin{array}{l}\text { Inlet } \\
\text { velocity } \\
\text { (Reynolds } \\
\text { number) }\end{array}$ & Remarks \\
\hline 34 & $\begin{array}{c}\text { Nuntaphan et al } \\
\text { (2005a) }\end{array}$ & $17.3-27.2$ & 4 & $\begin{array}{c}50-84 / \\
24.2-50\end{array}$ & $\begin{array}{l}\text { Crimped } \\
\text { spiral }\end{array}$ & $\begin{array}{c}37.3 \\
57.2 / 0.4\end{array}$ & $\begin{array}{c}2.85- \\
6.10\end{array}$ & $\begin{array}{c}\text { /Hot water } \\
\text { (65) }\end{array}$ & $\begin{array}{l}0.6-1.7 \\
(600- \\
2700)\end{array}$ & $\begin{array}{l}\text { The effect of the tube diameter, fin height, } \\
\text { and the fin spacing was studied for the } \\
\text { staggered and the inline arrangement of the } \\
\text { crimped-spiral-finned tubes. }\end{array}$ \\
\hline 35 & $\begin{array}{l}\text { Nuntaphan et al } \\
\quad(2005 b)\end{array}$ & $17.3-27.2$ & 4 & $\begin{array}{c}50- \\
84 / 24.2- \\
50\end{array}$ & $\begin{array}{l}\text { Crimped } \\
\text { spiral }\end{array}$ & $\begin{array}{c}37.3,57.2 / \\
0.4\end{array}$ & $\begin{array}{c}3.25- \\
6.50\end{array}$ & $\begin{array}{l}\text { /Cold water } \\
\text { (65) }\end{array}$ & $\begin{array}{l}0.6-1.7 \\
(600- \\
2700)\end{array}$ & $\begin{array}{l}\text { The effect of the tube diameter, fin height, } \\
\text { and the fin spacing was studied for the } \\
\text { staggered and the inline arrangement of the } \\
\text { crimped-spiral-finned tubes in the wet } \\
\text { conditions. }\end{array}$ \\
\hline 36 & $\begin{array}{c}\text { Kawaguchi et al } \\
\text { (2004) }\end{array}$ & 17.3 & $3-6$ & $\begin{array}{c}40- \\
45 / 30-40\end{array}$ & $\begin{array}{l}\text { Spiral } \\
\text { annular } \\
\text { and } \\
\text { serrated }\end{array}$ & $\begin{array}{l}35.3 / \\
0.9\end{array}$ & $3.3-5$ & $\begin{array}{l}\text { /Hot water } \\
\text { (60) }\end{array}$ & $\begin{array}{l}(2000- \\
27000 \\
R e_{h}\end{array}$ & $\begin{array}{l}\text { The thermal-hydraulic performance of the } \\
\text { serrated fin and the spiral-annular fin was } \\
\text { compared. }\end{array}$ \\
\hline 37 & $\begin{array}{l}\text { Kwak et al } \\
\quad(2005)\end{array}$ & 30 & 3 & $75 / 75$ & $\begin{array}{l}\text { Plate } \\
\text { with } \\
\text { DWP }\end{array}$ & & 5.6 & $\begin{array}{c}0.15 \times 0.1 \times \\
0.3 / \\
\text { Electrical } \\
\text { heating }\end{array}$ & $\begin{array}{c}0.5-3.5 \\
(937- \\
\left.6428, R e_{f r}\right)\end{array}$ & $\begin{array}{l}\text { The inline arrangement performed better } \\
\text { than the staggered arrangement for the two } \\
\text { rows of DWP in the common flow up } \\
\text { configuration. }\end{array}$ \\
\hline 38 & $\begin{array}{l}\text { Wongwises } \\
\quad \& \\
\text { Chokeman } \\
(2005)\end{array}$ & 9.53 & $2-6$ & $\begin{array}{c}25.4 / 19.0 \\
5\end{array}$ & Wavy & $\begin{array}{l}/ 0.115- \\
0.250\end{array}$ & $\begin{array}{l}1.41- \\
2.54\end{array}$ & $\begin{array}{l}0.43 \times 0.48 / \\
\text { Hot water } \\
(55-65)\end{array}$ & $\begin{array}{l}1-6(900- \\
5600)\end{array}$ & $\begin{array}{l}\text { The effect of fin pitch, number of tube rows } \\
\text { and the Reynolds number on the } \\
\text { performance of wavy finned-tube heat } \\
\text { exchanger was studied. }\end{array}$ \\
\hline 39 & $\begin{array}{l}\text { Chokeman } \\
\quad \& \\
\text { Wongwises } \\
\text { (2005) }\end{array}$ & 9.53 & 2 & $25.4 / 22$ & Wavy & $/ 0.115$ & 1.81 & $\begin{array}{l}0.43 \times 0.48 / \\
\text { Hot water } \\
(55-65)\end{array}$ & $\begin{array}{c}1.4-6 \\
(1400- \\
5600)\end{array}$ & $\begin{array}{l}\text { The fin pattern and edge corrugation } \\
\text { affected the performance of the heat } \\
\text { exchangers significantly. }\end{array}$ \\
\hline
\end{tabular}


Table 1. (contd)

\begin{tabular}{|c|c|c|c|c|c|c|c|c|c|c|}
\hline $\begin{array}{l}\text { Sr. } \\
\text { no. }\end{array}$ & $\begin{array}{l}\text { Author/s } \\
\text { and year }\end{array}$ & \multicolumn{3}{|c|}{ Design of the tube assembly } & \multicolumn{3}{|c|}{ Design of the fins } & $\begin{array}{c}\text { Geometry of } \\
\text { the shell } \\
\left(\mathrm{m}^{3} \text { or } \mathrm{m}^{2}\right) / \\
\text { thermal source } \\
\left({ }^{\circ} \mathrm{C} \text { or } \mathrm{W}\right)\end{array}$ & $\begin{array}{c}\text { Inlet } \\
\text { velocity } \\
\text { (Reynolds } \\
\text { number) }\end{array}$ & Remarks \\
\hline 40 & $\begin{array}{l}\text { Pirompugd et al } \\
\text { (2005) }\end{array}$ & $\begin{array}{l}8.51- \\
10.34\end{array}$ & $2-6$ & $\begin{array}{c}25.4 / 19.0 \\
5-22\end{array}$ & Plate & $\begin{array}{l}/ 0.115- \\
0.130\end{array}$ & $\begin{array}{c}1.195- \\
3.16\end{array}$ & $\begin{array}{l}0.85 \times 0.55 / \\
\quad \text { Cold } \\
\text { water }(7)\end{array}$ & $\begin{array}{l}0.3-4.5 \\
(300- \\
5500)\end{array}$ & $\begin{array}{l}\text { The effect of the inlet conditions and } \\
\text { geometric parameters on the heat and mass } \\
\text { transfer was observed for } 1 \text { and } 2 \text { row coils } \\
\text { in wet conditions. }\end{array}$ \\
\hline 41 & $\begin{array}{l}\text { Naphon } \\
\quad \& \\
\text { Wongwises } \\
(2005)\end{array}$ & $\begin{array}{l}9.6 \\
\text { (spiral } \\
\text { coiled } \\
\text { tubes) }\end{array}$ & 6 & & $\begin{array}{l}\text { Crimped } \\
\text { spiral }\end{array}$ & $\begin{array}{l}28.2 / \\
0.35\end{array}$ & 3.1 & $\begin{array}{c}300 \mathrm{~mm} \\
\text { diameter } \\
\text { (cylindrical } \\
\text { tunnel) } \\
\text { /Water, wet } \\
\text { conditions } \\
(12.5), \text { dry } \\
\text { conditions } \\
(30)\end{array}$ & & $\begin{array}{l}\text { The effect of the inlet air temperature, air } \\
\text { mass flow rate, water inlet temperature, and } \\
\text { the water flow rate was described on the } \\
\text { heat transfer performance. }\end{array}$ \\
\hline 42 & $\begin{array}{l}\text { Chen et al } \\
\text { (2005) }\end{array}$ & 40 & 1 & & Plate & $\begin{array}{c}100 \times 100 / \\
0.2\end{array}$ & - & $\begin{array}{l}\text { /Hot and } \\
\text { cold water } \\
\text { ( } 75 \text { and } 7)\end{array}$ & $\begin{array}{c}0.3-6.5 \\
(2500 \text { to } \\
13000 \\
\left.R e_{f r}\right)\end{array}$ & $\begin{array}{l}\text { The average heat transfer increased and the } \\
\text { fin efficiency decreased with an increase in } \\
\text { the base to ambient temperature difference } \\
\text { and inlet air velocity. }\end{array}$ \\
\hline 43 & $\begin{array}{l}\text { Pesteei } \text { et al } \\
\quad(2005)\end{array}$ & 50.8 & 1 & & $\begin{array}{l}\text { Plate } \\
\text { with } \\
\text { DWP }\end{array}$ & $300 \times 204 / 3$ & 15 & $\begin{array}{c}300 \times 300 \times \\
600 / \\
\text { Electrical } \\
\text { heating }\end{array}$ & (9525) & $\begin{array}{l}\text { The best location for the DWP was at } \\
\Delta x=0.5 D \text { and } \Delta y=0.5 D \text {, where } \Delta x \text { and } \Delta y \\
\text { were the streamwise and cross-stream } \\
\text { distances. }\end{array}$ \\
\hline 44 & $\begin{array}{l}\text { Pirompugd et al } \\
\quad(2006)\end{array}$ & $\begin{array}{l}8.62- \\
10.38\end{array}$ & $1-6$ & $\begin{array}{c}25.4 / 19.6 \\
3-26.27\end{array}$ & Wavy & $/ 0.12$ & $\begin{array}{l}1.57- \\
3.63\end{array}$ & $\begin{array}{l}0.85 \times 0.55 / \\
\text { Hot water } \\
\quad(7)\end{array}$ & $\begin{array}{l}0.3-3.8 \\
(400- \\
5000)\end{array}$ & $\begin{array}{l}\text { The ratio of the heat transfer to the mass } \\
\text { transfer coefficient varied from } 0.6 \text { to } 1.1 \text {, } \\
\text { and was insensitive to the fin spacing. }\end{array}$ \\
\hline
\end{tabular}


Table 1. (contd)

\begin{tabular}{|c|c|c|c|c|c|c|c|c|c|c|}
\hline \multirow{2}{*}{$\begin{array}{l}\text { Sr. } \\
\text { no. }\end{array}$} & \multirow{2}{*}{$\begin{array}{l}\text { Author/s } \\
\text { and year }\end{array}$} & \multicolumn{3}{|c|}{ Design of the tube assembly } & \multicolumn{3}{|c|}{ Design of the fins } & \multirow[b]{2}{*}{$\begin{array}{l}\text { Geometry of } \\
\text { the shell } \\
\left(\mathrm{m}^{3} \text { or } \mathrm{m}^{2}\right) / \\
\text { thermal source } \\
\left({ }^{\circ} \mathrm{C} \text { or } \mathrm{W}\right)\end{array}$} & \multirow[b]{2}{*}{$\begin{array}{l}\text { Inlet } \\
\text { velocity } \\
\text { (Reynolds } \\
\text { number) }\end{array}$} & \multirow[t]{2}{*}{ Remarks } \\
\hline & & $\begin{array}{l}\text { Diameter } \\
\text { of the } \\
\text { tubes, } \\
D(\mathrm{~mm})\end{array}$ & $\begin{array}{l}\text { Number } \\
\text { of rows, } \\
\quad N_{r}\end{array}$ & $\begin{array}{c}\text { Tube } \\
\text { pitch } \\
\left(S_{t}(\mathrm{~mm}) /\right. \\
\left.S_{l}(\mathrm{~mm})\right)\end{array}$ & $\begin{array}{l}\text { Type of } \\
\text { fins }\end{array}$ & $\begin{array}{l}\text { Length or } \\
\text { diameter } \\
(\mathrm{mm}) / \\
\text { thickness, } \\
t_{f}(\mathrm{~mm})\end{array}$ & $\begin{array}{c}\text { Pitch, } \\
S_{f}(\mathrm{~mm})\end{array}$ & & & \\
\hline 45 & $\begin{array}{l}\text { Wongwises } \\
\quad \& \\
\text { Naphon } \\
(2006 \mathrm{a})\end{array}$ & $\begin{array}{l}9.6 \\
\text { (spiral } \\
\text { coiled } \\
\text { tubes) }\end{array}$ & 6 & & $\begin{array}{c}\text { Crimped } \\
\text { spiral }\end{array}$ & $\begin{array}{l}28.2 / \\
0.35\end{array}$ & 3.1 & $\begin{array}{l}300 \mathrm{~mm} \\
\text { diameter } \\
\text { (cylindrical } \\
\text { tunnel)/ } \\
\text { Cold Water } \\
(7.5-20)\end{array}$ & & $\begin{array}{l}\text { The enthalpy effectiveness and humidity } \\
\text { effectiveness were defined and their } \\
\text { behaviour was also described. The liquid } \\
\text { film had significant effect on the heat } \\
\text { transfer performance of the heat exchanger. }\end{array}$ \\
\hline 46 & $\begin{array}{l}\text { Wongwises } \\
\quad \& \\
\text { Naphon } \\
(2006 b)\end{array}$ & $\begin{array}{l}9.6 \\
\text { (spiral } \\
\text { coiled } \\
\text { tubes) }\end{array}$ & 6 & & $\begin{array}{c}\text { Crimped } \\
\text { spiral }\end{array}$ & $\begin{array}{l}28.2 / \\
0.35\end{array}$ & 3.1 & $\begin{array}{l}300 \mathrm{~mm} \\
\text { diameter } \\
\text { (cylindrical } \\
\text { tunnel)/ } \\
\text { Cold Water } \\
(30-35)\end{array}$ & & \\
\hline 48 & $\begin{array}{l}\text { Chen et al } \\
\text { (2007) }\end{array}$ & 27.3 & 1 & & Plate & $\begin{array}{c}100 \times 100 / \\
0.1\end{array}$ & $5.1-50$ & $\begin{array}{c}0.2 \times 0.2 / \\
\text { Electrical } \\
\text { heating }\end{array}$ & $\begin{array}{c}1-5 \\
(1550- \\
\left.7760, R e_{f r}\right)\end{array}$ & $\begin{array}{l}\text { The fin efficiency decreased and the heat } \\
\text { transfer coefficient increased with an } \\
\text { increase in the air velocity and base to } \\
\text { ambient temperature difference. }\end{array}$ \\
\hline 49 & $\begin{array}{l}\text { Jaordar } \\
\text { \& Jacobi } \\
\text { (2008) }\end{array}$ & & & & & & & $\begin{array}{l}0.101 \times 0.610 / \\
\text { Electrical } \\
\text { heating }\end{array}$ & $\begin{array}{c}0.7-1.8 \\
(220-960 \\
\left.R e_{h}\right)\end{array}$ & $\begin{array}{l}\text { The } j / f \text { factor was higher ( } 35.7 \% \text { to } 50.8 \% \text { ) } \\
\text { for the one row of the DWP as compared to } \\
\text { the three rows of the DWP. However, the } \\
\text { volume goodness factor was better for the } \\
\text { three rows of the DWP as compared to the } \\
\text { one row of the DWP. }\end{array}$ \\
\hline
\end{tabular}


Table 1. (contd)

\begin{tabular}{|c|c|c|c|c|c|c|c|c|c|c|}
\hline $\begin{array}{l}\text { Sr. } \\
\text { no. }\end{array}$ & $\begin{array}{l}\text { Author/s } \\
\text { and year }\end{array}$ & \multicolumn{3}{|c|}{ Design of the tube assembly } & \multicolumn{3}{|c|}{ Design of the fins } & $\begin{array}{l}\text { Geometry of } \\
\text { the shell } \\
\left(\mathrm{m}^{3} \text { or } \mathrm{m}^{2}\right) / \\
\text { thermal source } \\
\left({ }^{\circ} \mathrm{C} \text { or } \mathrm{W}\right)\end{array}$ & $\begin{array}{l}\text { Inlet } \\
\text { velocity } \\
\text { (Reynolds } \\
\text { number) }\end{array}$ & Remarks \\
\hline 50 & $\begin{array}{l}\text { Chen \& Hsu } \\
\text { (2008) }\end{array}$ & 27.3 & 1 & & Annular & $\begin{array}{l}99 / \\
0.1\end{array}$ & $5.1-50$ & $\begin{array}{c}0.2 \times 0.2 / \\
\text { Electrical } \\
\text { heating }\end{array}$ & $\begin{array}{c}1-5 \\
(1550- \\
\left.7760, R e_{f r}\right)\end{array}$ & \\
\hline 51 & $\begin{array}{l}\text { Pirompugd et al } \\
\quad(2007 \mathrm{a}, \mathrm{b})\end{array}$ & $\begin{array}{l}8.51- \\
10.23\end{array}$ & $1-6$ & $\begin{array}{c}25.4 / 12.7 \\
22.0\end{array}$ & Plate & $\begin{array}{c}/ 0.115- \\
0.130\end{array}$ & $\begin{array}{l}1.315- \\
3.33\end{array}$ & $\begin{array}{l}0.85 \times 0.55 / \\
\text { Hot water } \\
\quad(7)\end{array}$ & $\begin{array}{c}0.3-3.8 \\
(525- \\
7650)\end{array}$ & $\begin{array}{l}\text { A new mathematical model [FCFM (Finite } \\
\text { circular fin method)] was developed to } \\
\text { determine the performance of the plain } \\
\text { finned-tube heat exchangers under wet } \\
\text { surfaces condition. }\end{array}$ \\
\hline 52 & $\begin{array}{c}\text { Saechan and } \\
\text { Wongwises (2008) }\end{array}$ & 9.53 & $2-5$ & & Plate & $/ 0.3$ & 6.6 & & $\begin{array}{c}(940- \\
20000)\end{array}$ & $\begin{array}{l}\text { The second law of thermodynamics was } \\
\text { used to optimize the performance of the } \\
\text { plain-finned-tube heat exchanger. }\end{array}$ \\
\hline 54 & $\begin{array}{l}\text { Paeng et al } \\
\quad \text { (2010) }\end{array}$ & 10.2 & 3 & $25 / 22$ & Plate & $/ 0.33$ & 3.2 & $\begin{array}{c}0.36 \times 0.27 \times \\
1.5 / \\
\mathrm{R}-22\end{array}$ & $\begin{array}{c}1.13-1.61 \\
(1082- \\
1649)\end{array}$ & $\begin{array}{l}\text { The correlation for the Nusselt number was } \\
\text { derived, and the error between the numerical } \\
\text { and the experimental results was } 6 \% \text {. }\end{array}$ \\
\hline 55 & $\begin{array}{l}\text { Huang et al } \\
\text { (2009) }\end{array}$ & 25.4 & 3 & $\begin{array}{c}52.6 \\
60.7 / 60.7\end{array}$ & Plate & $196 \times 240 / 1$ & 10-15 & $\begin{array}{l}0.3 \times 0.3 / \\
\text { Electrical } \\
\text { heating }\end{array}$ & $0.5-1.5$ & $\begin{array}{l}\text { The heat transfer coefficient for the } \\
\text { staggered arrangement was higher than the } \\
\text { heat transfer coefficient for the inline } \\
\text { arrangement. }\end{array}$ \\
\hline
\end{tabular}


Table 1. (contd)

\begin{tabular}{|c|c|c|c|c|c|c|c|c|c|c|}
\hline \multirow{2}{*}{$\begin{array}{l}\text { Sr. } \\
\text { no. }\end{array}$} & \multirow{2}{*}{$\begin{array}{l}\text { Author/s } \\
\text { and year }\end{array}$} & \multicolumn{3}{|c|}{ Design of the tube assembly } & \multicolumn{3}{|c|}{ Design of the fins } & \multirow[b]{2}{*}{$\begin{array}{l}\text { Geometry of } \\
\text { the shell } \\
\left(\mathrm{m}^{3} \text { or } \mathrm{m}^{2}\right) / \\
\text { thermal source } \\
\left({ }^{\circ} \mathrm{C} \text { or } \mathrm{W}\right)\end{array}$} & \multirow[b]{2}{*}{$\begin{array}{l}\text { Inlet } \\
\text { velocity } \\
\text { (Reynolds } \\
\text { number) }\end{array}$} & \multirow[t]{2}{*}{ Remarks } \\
\hline & & $\begin{array}{l}\text { Diameter } \\
\text { of the } \\
\text { tubes, } \\
D(\mathrm{~mm})\end{array}$ & $\begin{array}{l}\text { Number } \\
\text { of rows, } \\
N_{r}\end{array}$ & $\begin{array}{c}\text { Tube } \\
\text { pitch } \\
\left(S_{t}(\mathrm{~mm}) /\right. \\
\left.S_{l}(\mathrm{~mm})\right)\end{array}$ & $\begin{array}{l}\text { Type of } \\
\text { fins }\end{array}$ & $\begin{array}{l}\text { Length or } \\
\text { diameter } \\
(\mathrm{mm}) / \\
\text { thickness, } \\
t_{f}(\mathrm{~mm})\end{array}$ & $\begin{array}{c}\text { Pitch, } \\
S_{f}(\mathrm{~mm})\end{array}$ & & & \\
\hline 56 & $\begin{array}{l}\text { Ibrahim } \\
\& \\
\text { Gomaa } \\
(2009)\end{array}$ & $\begin{array}{l}12.7 \\
\text { (circular), } \\
5.2 \times 7.8- \\
3.2 \times 12.7 \\
\text { (elliptic } \\
\text { tubes) }\end{array}$ & 5 & $30 / 26$ & & & & $\begin{array}{c}\text { /Hot water, } \\
80\end{array}$ & $\begin{array}{c}3.8-20 \\
(5300- \\
28000 \\
\left.R e_{h}\right)\end{array}$ & $\begin{array}{l}\text { Four criterions for the thermal performance } \\
\text { were identified, (1) comparison of heat } \\
\text { transfer and pressure drop (2) heat transfer } \\
\text { per unit pressure drop (3) area goodness } \\
\text { factor and (4) efficiency index. }\end{array}$ \\
\hline 57 & $\begin{array}{l}\text { Tang et al } \\
\text { (2009a) }\end{array}$ & 18 & $6-12$ & $42 / 34$ & $\begin{array}{l}\text { Plain, } \\
\text { slit, } \\
\text { vortex }\end{array}$ & $/ 0.3$ & 3.1 & $\begin{array}{l}\text { /Superheated } \\
\text { steam } \\
(65)\end{array}$ & $\begin{array}{l}2.5-5 \\
(3870- \\
9677)\end{array}$ & $\begin{array}{l}\text { The } j / f \text { ratio for the slit fin increased at a } \\
\text { higher rate as compared to the plain fin and } \\
\text { fin with DWP. }\end{array}$ \\
\hline 58 & $\begin{array}{l}\text { Tang et al } \\
\text { (2009b) }\end{array}$ & 18 & $6-12$ & $42 / 34$ & $\begin{array}{c}\text { Crimped } \\
\text { Spiral, } \\
\text { Plain, } \\
\text { slit, Plate } \\
\text { with DWP, } \\
\text { mixed } \\
\text { (DWP + } \\
\text { slit) }\end{array}$ & $/ 0.3$ & 3.1 & $\begin{array}{l}\text { /Superheated } \\
\text { steam } \\
(65)\end{array}$ & $\begin{array}{l}2.5-5 \\
(3870- \\
9677)\end{array}$ & $\begin{array}{l}\text { After optimization, it was found that the } \\
\text { fin with DWP could perform better } \\
\text { than the slit fin. }\end{array}$ \\
\hline 59 & $\begin{array}{l}\text { Choi et al } \\
\text { (2010) }\end{array}$ & 8 & $1-4$ & $26 / 28-34$ & $\begin{array}{l}\text { Discrete } \\
\text { plate }\end{array}$ & $62 \times 27 /$ & $7.5-15$ & $\begin{array}{l}1.4 \times 0.4 \times \\
(0.44- \\
0.6) / \text { Ethylene } \\
\text { glycol-water } \\
\text { mixture, } 33\end{array}$ & $\begin{array}{c}1-1.65 \\
(500-800)\end{array}$ & $\begin{array}{l}\text { The Colburn factor for the discrete plate } \\
\text { finned-tube was found to be } 6-11 \% \text { more } \\
\text { than the continuous plate finned-tube heat } \\
\text { exchanger for a fin pitch of } 7.5-15 \mathrm{~mm} \text {. }\end{array}$ \\
\hline 60 & Næss (2010) & $\begin{array}{l}19.07- \\
31.77\end{array}$ & 4 & $\begin{array}{l}46.1- \\
79.8 / 23.1- \\
50.9\end{array}$ & Serrated & $/ 0.91$ & $\begin{array}{c}3.62- \\
5.08\end{array}$ & $\begin{array}{c}\text { /Water- } \\
\text { glycol }\end{array}$ & $\begin{array}{l}(2282- \\
47290)\end{array}$ & $\begin{array}{l}\text { It was found that the Nusselt number } \\
\text { shows a maxima when the flow areas } \\
\text { in the transverse and diagonal directions } \\
\text { become equal. }\end{array}$ \\
\hline
\end{tabular}


Table 1. (contd)

\begin{tabular}{|c|c|c|c|c|c|c|c|c|c|c|}
\hline $\begin{array}{l}\text { Sr. } \\
\text { no. }\end{array}$ & $\begin{array}{l}\text { Author/s } \\
\text { and year }\end{array}$ & \multicolumn{3}{|c|}{ Design of the tube assembly } & \multicolumn{3}{|c|}{ Design of the fins } & $\begin{array}{l}\text { Geometry of } \\
\text { the shell } \\
\left(\mathrm{m}^{3} \text { or } \mathrm{m}^{2}\right) / \\
\text { thermal source } \\
\left({ }^{\circ} \mathrm{C} \text { or } \mathrm{W}\right)\end{array}$ & $\begin{array}{l}\text { Inlet } \\
\text { velocity } \\
\text { (Reynolds } \\
\text { number) }\end{array}$ & Remarks \\
\hline 61 & $\begin{array}{l}\text { Martinez et al } \\
\quad(2010)\end{array}$ & 50.8 & 8 & $\begin{array}{l}114.3 / \\
99.06\end{array}$ & Serrated & $101.6 / 1.24$ & 4.23 & $\begin{array}{l}1.4 \times 0.8-1.4 \\
\times 0.8 / \text { Water } \\
(105-108)\end{array}$ & $\begin{array}{l}(6200- \\
11200)\end{array}$ & $\begin{array}{l}\text { Various correlation for the heat transfer } \\
\text { and pressure drop performance of the } \\
\text { serrated fins were compared. }\end{array}$ \\
\hline 63 & $\begin{array}{l}\text { Pongsoi et al } \\
\quad \text { (2011) }\end{array}$ & 16.35 & 2 & $39 / 35$ & $\begin{array}{l}\text { Crimped } \\
\text { spiral }\end{array}$ & $34.8 / 0.4$ & $3.2-6.2$ & $\begin{array}{c}0.43 \times \\
0.48 / \mathrm{Hot} \\
\text { water }(60)\end{array}$ & $\begin{array}{c}2-6 \\
(4000- \\
13000)\end{array}$ & $\begin{array}{l}\text { It was found that, the effect of fin pitch } \\
\text { on the Colburn factor was very small for } \\
2 \text { row coil due to better mixing at high Re } \\
\text { number. }\end{array}$ \\
\hline 65 & $\begin{array}{l}\text { Chen \& Lai } \\
\text { (2012) }\end{array}$ & 27 & 2 & $\begin{array}{l}214.28 / \\
214.28\end{array}$ & Plate & $\begin{array}{l}126 \times \\
126 / 1\end{array}$ & $5-50$ & $\begin{array}{c}0.22 \times \\
0.22 / \text { Electrical } \\
\text { heating }\end{array}$ & $\begin{array}{l}0.5-1.5 \\
(816- \\
2500)\end{array}$ & $\begin{array}{l}\text { The heat transfer coefficient increased } \\
\text { with an increase in the air velocity and } \\
\text { fin spacing, however, it reached its asymptotic } \\
\text { value as the fin spacing approached infinity. }\end{array}$ \\
\hline 66 & $\begin{array}{l}\text { Pongsoi et al } \\
(2012 \mathrm{a}, \mathrm{b}, \mathrm{c})\end{array}$ & 16.35 & $2-5$ & $40 / 35$ & $\begin{array}{l}\text { Crimped } \\
\text { spiral }\end{array}$ & $35 / 0.5$ & 6.3 & $\begin{array}{c}0.43 \times \\
0.48 / \text { Hot } \\
\text { water }(60-65)\end{array}$ & $\begin{array}{c}2-6 \\
(4000- \\
13000)\end{array}$ & $\begin{array}{l}\text { It was found that, the number of tube rows } \\
\text { does not affect the Colburn factor and the } \\
\text { friction factor beyond a Re number of } 2000 \text {. } \\
\text { This was believed to be due to the shedding } \\
\text { of the downstream turbulence eddies, which } \\
\text { causes good mixing. }\end{array}$ \\
\hline
\end{tabular}


Table 1. (contd)

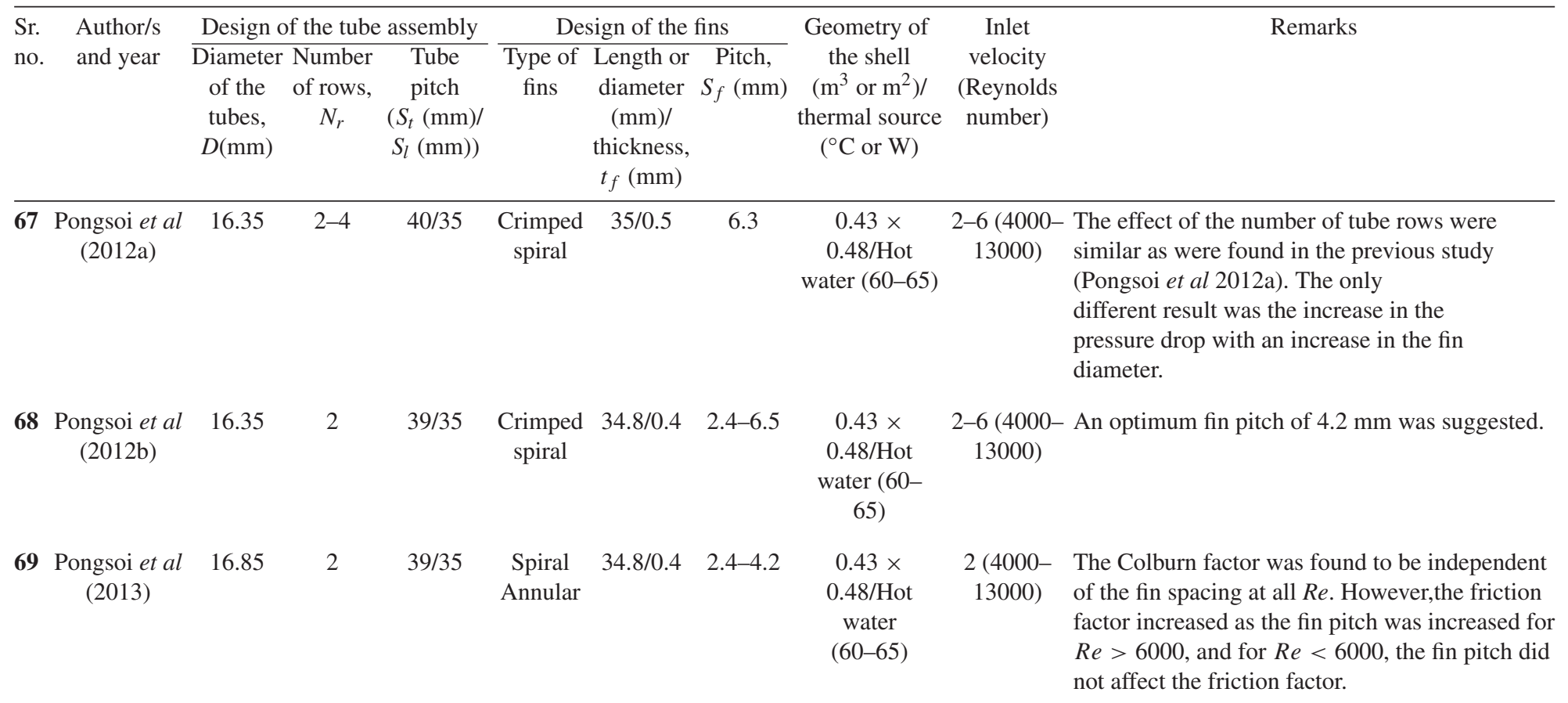


Table 2. Summary of published work (numerical).

\begin{tabular}{|c|c|c|c|c|c|c|c|c|c|c|}
\hline Sr. & Author/s & & Computatic & onal dom & Iain & Thermal & $R e$ & Grid & Models/ & Remarks \\
\hline no. & and year & $\begin{array}{c}\text { Diameter Number } \\
\text { of the of rows, } \\
\text { tubes, } \quad N_{r} \\
D(\mathrm{~mm})\end{array}$ & $\begin{array}{c}\text { Tube } \\
\text { pitch } \\
\left(S_{t}(\mathrm{~mm}) /\right. \\
\left.S_{l}(\mathrm{~mm})\right)\end{array}$ & $\begin{array}{l}\text { Type of } \\
\text { fins }\end{array}$ & 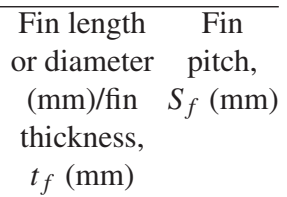 & $\begin{array}{c}\mathrm{BC}(\mathrm{Q}(\mathrm{W}), \\
\mathrm{T}(\mathrm{K}))\end{array}$ & & $\begin{array}{c}\text { size/ } \\
\text { scheme }\end{array}$ & $\begin{array}{l}\text { solution } \\
\text { algorithm }\end{array}$ & \\
\hline
\end{tabular}

1. Brockmeier

et al (1993)

2. Biswas et al

(1994)

3. Fiebig et al

(1995)

4. Rocha et al

(1997)

5. Tsai \& Sheu 7.5 (1998)

Plain
with
DWP

DWP

$t_{f}(\mathrm{~mm})$

The vortices were observed near the leading edge of the winglet. The fin with DWP allowed a $76 \%$ decrease in the heat transfer area as

compared to the plain fins.

temperature $3000, \quad \times 20$

for fin and $R e_{h}$

$$
\text { tube }
$$

\begin{tabular}{|c|c|c|c|c|}
\hline $\begin{array}{l}\text { Constant } \\
\text { temperature } \\
\text { for fin and } \\
\text { tube }\end{array}$ & $\begin{array}{c}500- \\
1000, \\
R e_{s}\end{array}$ & $\begin{array}{c}98 \times 14 \\
\times 34\end{array}$ & $\begin{array}{c}\text { /Marker } \\
\text { and Cell } \\
(\mathrm{MAC})\end{array}$ & $\begin{array}{l}\text { The interaction of the } \\
\text { longitudinal and transverse } \\
\text { vortices led to the periodic } \\
\text { flow for the } \operatorname{Re}=1000 \text {. }\end{array}$ \\
\hline
\end{tabular}

Constant 250-

temperature 300 ,

for the tube $R e_{S}$

/SIMPLEC The DWP reduced the heat transfer reversal and enhanced the heat transfer behind the tubes in the wake zone.

The elliptical arrangement performed better than the circular arrangement with $18 \%$ more efficiency.

Plain

The horseshoe vortex led to higher heat transfer in the upstream of the tubes. The wake region behind the second row tubes gave rise to heat transfer reversal (HTR). 
Table 2. (contd)

\begin{tabular}{|c|c|c|c|c|c|c|c|c|c|c|c|c|}
\hline $\begin{array}{l}\text { Sr. } \\
\text { no. }\end{array}$ & $\begin{array}{l}\text { Author/s } \\
\text { and year }\end{array}$ & \multicolumn{6}{|c|}{ Computational domain } & $\begin{array}{l}\text { Thermal } \\
\text { BC }(\mathrm{Q}(\mathrm{W}), \\
\mathrm{T}(\mathrm{K}))\end{array}$ & $R e$ & $\begin{array}{c}\text { Grid } \\
\text { size/ } \\
\text { scheme }\end{array}$ & $\begin{array}{l}\text { Models/ } \\
\text { solution } \\
\text { algorithm }\end{array}$ & Remarks \\
\hline 6. & $\begin{array}{l}\text { Chen et al } \\
\text { (1998a) }\end{array}$ & & 1 & & $\begin{array}{l}\text { Plain } \\
\text { with } \\
\text { DWP }\end{array}$ & & & $\begin{array}{l}\text { Constant } \\
\text { temperature } \\
\text { for tube }\end{array}$ & $\begin{array}{l}300, \\
R e_{s}\end{array}$ & $\begin{array}{c}187 \times 52 \\
\times 25-152 \\
\times 52 \times \\
25 / \text { Upwind } \\
\text { Scheme }\end{array}$ & $\begin{array}{l}\text { Laminar/ } \\
\text { SIMPLEC }\end{array}$ & $\begin{array}{l}\text { The best performance of } \\
\text { the delta winglets were } \\
\text { observed for an aspect ratio } \\
\text { of } 2 \text { and an attack angle of } \\
30^{\circ} \text {. }\end{array}$ \\
\hline 7. & $\begin{array}{l}\text { Chen et al } \\
\text { (1998b) }\end{array}$ & & 1 & & $\begin{array}{c}\text { Plain } \\
\text { with } \\
\text { DWP }\end{array}$ & & & $\begin{array}{l}\text { Constant } \\
\text { temperature } \\
\text { for tube }\end{array}$ & $\begin{array}{l}300, \\
R e_{s}\end{array}$ & $\begin{array}{l}152 \times 60 \\
\times 24 / \\
\text { Upwind } \\
\text { Scheme }\end{array}$ & $\begin{array}{l}\text { Laminar/ } \\
\text { SIMPLEC }\end{array}$ & $\begin{array}{l}\text { The heat transfer increased } \\
\text { with an increase in the } \\
\text { number of rows of delta } \\
\text { winglets. }\end{array}$ \\
\hline 8. & $\begin{array}{c}\text { Jang \& Yang } \\
\text { (1998) }\end{array}$ & $\begin{array}{c}36 \times 12.7 \\
\text { (elliptical) } \\
27.2\end{array}$ & 4 & $\begin{array}{l}50,37 / \\
34,42\end{array}$ & $\begin{array}{l}\text { Annular } \\
\text { and } \\
\text { elliptical }\end{array}$ & $\begin{array}{c}50 \times 26.7 \\
(\text { elliptical }) \\
41 / 0.5\end{array}$ & 3.175 & $\begin{array}{l}\text { Constant } \\
\text { temperature } \\
\text { without } \\
\text { conjugate } \\
\text { heat } \\
\text { transfer }\end{array}$ & $\begin{array}{l}20000- \\
80000 \\
R e_{p}\end{array}$ & $\begin{array}{l}10 \times 12 \\
\times 178 \\
-9 \times 11 \\
\times 152 / \\
\text { Hybrid } \\
\text { Scheme }\end{array}$ & $\begin{array}{l}\text { Laminar/ } \\
\text { SIMPLER }\end{array}$ & $\begin{array}{l}\text { The heat transfer per unit } \\
\text { pressure drop was } 50 \% \\
\text { higher for the elliptical } \\
\text { finned tubes as compared } \\
\text { to the circular finned tubes. }\end{array}$ \\
\hline 9. & $\begin{array}{l}\text { Sheu \& Tsai } \\
\text { (1999) }\end{array}$ & 7.5 & 2 & $\begin{array}{c}12.75 / \\
20.4\end{array}$ & $\begin{array}{c}\text { Plain and } \\
\text { slit }\end{array}$ & $\begin{array}{c}25.5 \times \\
20.4 / 0.0575\end{array}$ & 1.4 & $\begin{array}{l}\text { Constant } \\
\text { temperature } \\
\text { at tube (50) }\end{array}$ & $\begin{array}{c}367.28- \\
1133.594 \\
R e_{h}\end{array}$ & $\begin{array}{c}142 \times 84 \\
\times 54 / \\
\text { QUICK }\end{array}$ & $\begin{array}{l}\text { Laminar/ } \\
\text { SIMPLE }\end{array}$ & $\begin{array}{l}\text { The heat transfer was } \\
\text { higher near the leading } \\
\text { edge and upstream of the } \\
\text { tubes for both types of fins. } \\
\text { The Nu number decreased } \\
\text { along the flow direction. } \\
\text { The heat transfer and } \\
\text { pressure drop were higher } \\
\text { for the slit fin as compared } \\
\text { to the plate fin. }\end{array}$ \\
\hline
\end{tabular}


Table 2. (contd)

\begin{tabular}{|c|c|c|c|c|c|c|c|c|c|c|c|c|}
\hline \multirow[b]{2}{*}{$\begin{array}{l}\text { Sr. } \\
\text { no. } \\
\end{array}$} & \multirow{2}{*}{$\begin{array}{l}\text { Author/s } \\
\text { and year }\end{array}$} & \multicolumn{6}{|c|}{ Computational domain } & \multirow{2}{*}{$\begin{array}{l}\text { Thermal } \\
\mathrm{BC}(\mathrm{Q}(\mathrm{W}), \\
\mathrm{T}(\mathrm{K}))\end{array}$} & \multirow[t]{2}{*}{$\operatorname{Re}$} & \multirow{2}{*}{$\begin{array}{c}\text { Grid } \\
\text { size/ } \\
\text { scheme }\end{array}$} & \multirow{2}{*}{$\begin{array}{l}\text { Models/ } \\
\text { solution } \\
\text { algorithm }\end{array}$} & \multirow[t]{2}{*}{ Remarks } \\
\hline & & $\begin{array}{l}\text { Diameter } \\
\text { of the } \\
\text { tubes, } \\
D(\mathrm{~mm})\end{array}$ & $\begin{array}{l}\text { Number } \\
\text { of rows, } \\
N_{r}\end{array}$ & $\begin{array}{c}\text { Tube } \\
\text { pitch } \\
\left(S_{t}(\mathrm{~mm}) /\right. \\
\left.S_{l}(\mathrm{~mm})\right)\end{array}$ & $\begin{array}{l}\text { Type of } \\
\text { fins }\end{array}$ & $\begin{array}{c}\text { Fin length } \\
\text { or diameter } \\
(\mathrm{mm}) / \mathrm{fin} \\
\text { thickness, } \\
t_{f}(\mathrm{~mm})\end{array}$ & $\begin{array}{c}\text { Fin } \\
\text { pitch, } \\
S_{f}(\mathrm{~mm})\end{array}$ & & & & & \\
\hline 10. & $\begin{array}{l}\text { Tsai et al } \\
\text { (1998) }\end{array}$ & 7.5 & 2 & $\begin{array}{l}12.75 / \\
20.4\end{array}$ & $\begin{array}{l}\text { Plain and } \\
\text { Wavy }\end{array}$ & $\begin{array}{c}25.5 \times \\
20.4 / \\
0.0575\end{array}$ & 1.4 & $\begin{array}{l}\text { Constant } \\
\text { temperature } \\
\text { at tube }(50)\end{array}$ & $\begin{array}{c}367.28- \\
1133.594, \\
R e_{h}\end{array}$ & $\begin{aligned} & 142 \times 84 \\
& \times 54 \\
& 142 \times 84 \\
& \times 40 \\
& \text { QUICK }\end{aligned}$ & $\begin{array}{l}\text { Laminar/ } \\
\text { SIMPLE }\end{array}$ & $\begin{array}{l}\text { The heat transfer and } \\
\text { pressure drop were higher } \\
\text { for the wavy fin as } \\
\text { compared to the plate fin. }\end{array}$ \\
\hline 11. & $\begin{array}{l}\text { Chen et al } \\
\text { (2000) }\end{array}$ & & 1 & & Plain & & & $\begin{array}{l}\text { Constant } \\
\text { temperature } \\
\text { for tube }\end{array}$ & $\begin{array}{l}300 \\
R e_{s}\end{array}$ & $\begin{array}{c}152 \times 60 \\
\times 24\end{array}$ & /SIMPLEC & $\begin{array}{l}\text { The staggered arrangement } \\
\text { increased the heat transfer } \\
\text { by } 20 \% \text { and decreased the } \\
\text { pressure drop by } 14.6 \% \text { as } \\
\text { compared to the inline } \\
\text { arrangement. }\end{array}$ \\
\hline 12. & $\begin{array}{l}\text { omero-Mendez } \\
\text { et al (1997) }\end{array}$ & & 1 & & & & & $\begin{array}{l}\text { Constant } \\
\text { temperature } \\
\text { for tube } \\
\text { and fin }\end{array}$ & $\begin{array}{l}260- \\
1450\end{array}$ & & & $\begin{array}{l}\text { The Nusselt number per } \\
\text { unit pressure drop per unit } \\
\text { fin spacing was maximum } \\
\text { for an optimum fin spacing. }\end{array}$ \\
\hline 13. & $\begin{array}{l}\text { Matos et al } \\
\quad(2001)\end{array}$ & & 4 & & & & & $\begin{array}{l}\text { Constant } \\
\text { temperature }\end{array}$ & $\begin{array}{c}300- \\
800, \\
R e_{a} \\
\text { (based } \\
\text { on } \\
\text { array } \\
\text { length) }\end{array}$ & $\begin{array}{c}5180 \\
\text { (elements), } \\
\text { upwind }\end{array}$ & Laminar/ & $\begin{array}{l}\text { The elliptical tubes provided } \\
13 \% \text { more heat transfer as } \\
\text { compared to the circular } \\
\text { tubes. }\end{array}$ \\
\hline 14. & $\begin{array}{l}\text { Matos et al } \\
\text { (2004a) }\end{array}$ & & 4 & & & & & $\begin{array}{l}\text { Constant } \\
\text { temperature }\end{array}$ & $\begin{array}{c}300- \\
800 \\
R e_{a}\end{array}$ & $\begin{array}{c}5180 \\
\text { (elements), } \\
\text { upwind }\end{array}$ & Laminar/ & $\begin{array}{l}\text { The elliptical tubes provided } \\
\text { more heat transfer for a fixed } \\
\text { pressure drop. Dimensionless } \\
\text { tube spacing and eccentricity } \\
\text { were optimized. }\end{array}$ \\
\hline
\end{tabular}


Table 2. (contd)

\begin{tabular}{|c|c|c|c|c|c|c|c|c|c|c|c|c|}
\hline Sr. & Author/s & & & Computati & tional doma & & & Thermal & $R e$ & Grid & Models/ & Remarks \\
\hline no. & and year & $\begin{array}{c}\text { Diameter } \\
\text { of the } \\
\text { tubes, } \\
D(\mathrm{~mm})\end{array}$ & $\begin{array}{c}\text { Number } \\
\text { of rows, } \\
N_{r}\end{array}$ & $\begin{array}{c}\text { Tube } \\
\text { pitch } \\
\left(S_{t}(\mathrm{~mm}) /\right. \\
\left.S_{l}(\mathrm{~mm})\right)\end{array}$ & $\begin{array}{l}\text { Type of } \\
\text { fins }\end{array}$ & $\begin{array}{l}\text { Fin length } \\
\text { or diameter } \\
(\mathrm{mm}) / \mathrm{fin} \\
\text { thickness, } \\
t_{f}(\mathrm{~mm})\end{array}$ & $\begin{array}{c}\text { Fin } \\
\text { pitch, } \\
S_{f}(\mathrm{~mm})\end{array}$ & $\begin{array}{c}\mathrm{BC}(\mathrm{Q}(\mathrm{W}), \\
\mathrm{T}(\mathrm{K}))\end{array}$ & & $\begin{array}{c}\text { size/ } \\
\text { scheme }\end{array}$ & $\begin{array}{l}\text { solution } \\
\text { algorithm }\end{array}$ & \\
\hline 15. & $\begin{array}{l}\text { Matos et al } \\
\quad(2004 a)\end{array}$ & & 4 & & & & & $\begin{array}{l}\text { Constant } \\
\text { temperature }\end{array}$ & $\begin{array}{l}300- \\
800 \\
R e_{a}\end{array}$ & $\begin{array}{c}5180 \\
\text { (elements), } \\
\text { upwind }\end{array}$ & Laminar/ & $\begin{array}{l}\text { The optimum value of the } \\
\text { parameters [dimensionless } \\
\text { tube spacing }(0.5) \text {, eccentricity } \\
(0.5) \text { and dimensionless fin } \\
\text { spacing }(0.006) \text { ] were obtained. }\end{array}$ \\
\hline 16. & $\begin{array}{c}\text { Mon \& Gross } \\
\quad(2004)\end{array}$ & 24 & 4 & $\begin{array}{c}35.33- \\
45.73 / \\
40.8- \\
52.8\end{array}$ & Annular & $34 / 0.5$ & $0.7-4$ & $\begin{array}{l}\text { Constant } \\
\text { Temperature }\end{array}$ & $\begin{array}{l}8600- \\
43000\end{array}$ & $\begin{array}{l}50000- \\
90000, \\
\text { upwind }\end{array}$ & $\begin{array}{l}\mathrm{k}-\varepsilon / \\
\mathrm{PISO}\end{array}$ & $\begin{array}{l}\text { An optimized fin spacing was } \\
\text { obtained. The staggered } \\
\text { arrangement performed better } \\
\text { than the inline arrangement } \\
\text { of the tubes. }\end{array}$ \\
\hline 17. & $\begin{array}{c}\text { Cheng et al } \\
\text { (2004) }\end{array}$ & 19.1 & 3 & $25 / 25$ & $\begin{array}{c}\text { Plain and } \\
\text { slit }\end{array}$ & 0.3 & 2.5 & $\begin{array}{l}\text { Constant } \\
\text { temperature } \\
\text { for tube }\end{array}$ & $\begin{array}{l}288- \\
5000\end{array}$ & $\begin{array}{c}211 \times 85 \\
\times 24\end{array}$ & /CLEAR & $\begin{array}{l}\text { The slit fins performed better } \\
\text { than the plain fins, and the } \\
\text { increase in the heat transfer } \\
\text { was higher than the increase } \\
\text { in the pressure drop. }\end{array}$ \\
\hline 18. & $\begin{array}{l}\text { Qu et al } \\
\text { (2004) }\end{array}$ & 7.2 & 2 & $\begin{array}{l}12.7 / \\
11.97\end{array}$ & $\begin{array}{c}\text { Plain and } \\
\text { strip }\end{array}$ & 0.105 & 1.4 & $\begin{array}{l}\text { Constant } \\
\text { temperature } \\
\text { for tube }\end{array}$ & $\begin{array}{l}348- \\
3480\end{array}$ & $\begin{array}{c}136 \times \\
116 \times 34\end{array}$ & $\begin{array}{l}\text { Laminar/ } \\
\text { CLEAR }\end{array}$ & $\begin{array}{l}\text { The fin with strips in the } \\
\text { downstream part performed } \\
\text { best for velocity }<2 \mathrm{~m} / \mathrm{s} \text {. For } \\
u_{\mathrm{fr}}>2 \mathrm{~m} / \mathrm{s} \text {, the whole strip } \\
\text { fin performed best. }\end{array}$ \\
\hline
\end{tabular}


Table 2. (contd)

\begin{tabular}{|c|c|c|c|c|c|c|c|c|c|c|c|}
\hline \multirow{2}{*}{\multicolumn{2}{|c|}{$\begin{array}{ll}\text { Sr. } & \text { Author/s } \\
\text { no. } & \text { and year }\end{array}$}} & \multicolumn{6}{|c|}{ Computational domain } & \multirow{2}{*}{$\begin{array}{c}\text { Thermal } \\
\text { BC (Q (W), } \\
\mathrm{T}(\mathrm{K}))\end{array}$} & \multirow{2}{*}{ Re } & \multirow{2}{*}{$\begin{array}{c}\text { Grid } \\
\text { size/ } \\
\text { scheme }\end{array}$} & \multirow{2}{*}{$\begin{array}{l}\text { Models/ } \\
\text { solution } \\
\text { algorithm }\end{array}$} \\
\hline & & $\begin{array}{c}\text { Diameter } \\
\text { of the } \\
\text { tubes, } \\
D(\mathrm{~mm})\end{array}$ & $\begin{array}{c}\text { Number } \\
\text { of rows, } \\
N_{r}\end{array}$ & $\begin{array}{c}\text { Tube } \\
\text { pitch } \\
\left(S_{t}(\mathrm{~mm}) /\right. \\
\left.S_{l}(\mathrm{~mm})\right)\end{array}$ & $\begin{array}{l}\text { Type of } \\
\text { fins }\end{array}$ & $\begin{array}{l}\text { Fin length } \\
\text { or diameter } \\
(\mathrm{mm}) / \mathrm{fin} \\
\text { thickness, }\end{array}$ & $\begin{array}{c}\text { Fin } \\
\text { pitch, } \\
S_{f}(\mathrm{~mm})\end{array}$ & & & & \\
\hline
\end{tabular}

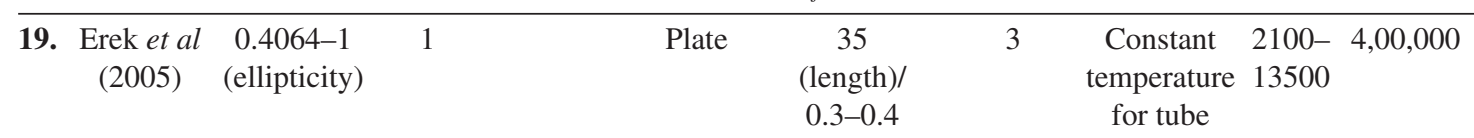

The heat transfer increased
with an increase in the fin
height, decrease in fin pitch,
increase in ellipticity, and decrease in tube thickness. The pressure drop increased with an increase in the fin spacing. 20. He et al 10

$\begin{array}{cr}1-4-30 / & \text { Plate } \quad \text { Length } \\ & =21.65- \\ & 86.65 \\ & \text { width }= \\ & 12.5 / 0.2\end{array}$

21. Lin \& Jang 20 (2005)
34.6 Plate $\quad$ Length $=138$, (staggered)

40

(inline)/

40

$$
140 \text {, }
$$

Width $=20 /$

0.2

0.5-5 Constant $500-62 \times 10 \quad$ /SIMPLER The synergy was worse at temperature $5000 \times 10-158$ for fin and $\quad \times 22$ tube

$$
\times 10
$$
the back side of the tube. Therefore improvement measurements were recommended for the backside of the tube.

The heat transfer enhancement by EHD was more effective at low Reynolds number and high applied voltage. The maximum heat transfer was obtained for a Reynolds number of 100 at $16 \mathrm{kV}$. 
Table 2. (contd)

\begin{tabular}{|c|c|c|c|c|c|c|c|c|c|c|c|c|}
\hline $\begin{array}{l}\text { Sr. } \\
\text { no. }\end{array}$ & $\begin{array}{l}\text { Author/s } \\
\text { and year }\end{array}$ & \multicolumn{6}{|c|}{ Computational domain } & $\begin{array}{l}\text { Thermal } \\
\mathrm{BC}(\mathrm{Q}(\mathrm{W}), \\
\mathrm{T}(\mathrm{K}))\end{array}$ & $\operatorname{Re}$ & $\begin{array}{c}\text { Grid } \\
\text { size/ } \\
\text { scheme }\end{array}$ & $\begin{array}{l}\text { Models/ } \\
\text { solution } \\
\text { algorithm }\end{array}$ & Remarks \\
\hline 22. & $\begin{array}{l}\text { Tao et al } \\
\text { (2006) }\end{array}$ & 8.31 & 2 & $\begin{array}{l}12.7 / \\
15.88\end{array}$ & $\begin{array}{l}\text { Slit fins } \\
\text { and Plate }\end{array}$ & $/ 0.114$ & & $\begin{array}{l}\text { Constant } \\
\text { temperature } \\
\text { for tube, } \\
283.15 \mathrm{~K}\end{array}$ & $\begin{array}{l}900- \\
2700\end{array}$ & $\begin{array}{c}143 \times 66 \\
\times 24 \\
\text { SGSD }\end{array}$ & /CLEAR & $\begin{array}{l}\text { Three different convergence } \\
\text { criterions were discussed. } \\
\text { Only one of them was found } \\
\text { to give the consistent results. }\end{array}$ \\
\hline 23. & $\begin{array}{l}\text { Jin et al } \\
(2006)\end{array}$ & 8.31 & 2 & $\begin{array}{l}12.7 / \\
15.88\end{array}$ & $\begin{array}{l}\text { Slit fins } \\
\text { and Plate }\end{array}$ & $/ 0.114$ & & $\begin{array}{l}\text { Constant } \\
\text { temperature } \\
\text { for tube, } \\
283.15 \mathrm{~K}\end{array}$ & $\begin{array}{l}900- \\
2700\end{array}$ & $\begin{array}{c}143 \times 66 \\
\times 24 \\
\text { SGSD }\end{array}$ & /CLEAR & $\begin{array}{l}\text { Optimum designs were } \\
\text { recommended for the slit fins. }\end{array}$ \\
\hline 24. & $\begin{array}{l}\text { Tao et al } \\
(2007 \mathrm{a})\end{array}$ & 10.55 & 2 & $\begin{array}{l}21.65 / \\
25\end{array}$ & Wavy & $\begin{array}{c}43.3 \times \\
12.5 / \\
0.2\end{array}$ & 2 & $\begin{array}{l}\text { Constant } \\
\text { temperature } \\
\text { for tube }\end{array}$ & $\begin{array}{l}500- \\
4000\end{array}$ & $\begin{array}{l}142 \times 22 \\
\times 12\end{array}$ & $\begin{array}{l}\text { Laminar/ } \\
\text { SIMPLE }\end{array}$ & $\begin{array}{l}\text { The heat transfer coefficient } \\
\text { increased and the fin efficiency } \\
\text { decreased along the air flow } \\
\text { direction. The wavy pattern } \\
\text { caused a fluctuating Nusselt } \\
\text { number distribution. }\end{array}$ \\
\hline 25. & $\begin{array}{l}\text { Tao et al } \\
\text { (2007b) }\end{array}$ & 19.1 & 3 & $25 / 25$ & $\begin{array}{l}\text { Slit and } \\
\text { Plate }\end{array}$ & $/ 0.3$ & 2.5 & $\begin{array}{l}\text { Constant } \\
\text { temperature, } \\
308\end{array}$ & $\begin{array}{l}650- \\
4800\end{array}$ & $\begin{array}{c}211 \times 85 \times \\
24 \\
\text { SGSD }\end{array}$ & $\begin{array}{l}\text { Laminar/ } \\
\text { CLEAR }\end{array}$ & $\begin{array}{l}\text { It was recommended that, } \\
\text { the strips should be placed } \\
\text { in a way that the thermal } \\
\text { resistance of the front and } \\
\text { the rear part becomes equal. }\end{array}$ \\
\hline 26. & $\begin{array}{l}\text { Cheng et al } \\
\quad(2007)\end{array}$ & $8.8-13.6$ & 2 & $22.4 / 25$ & Wavy & & $2-3$ & $\begin{array}{l}\text { Constant } \\
\text { temperature } \\
\text { for tube, } \\
\quad 303\end{array}$ & $\begin{array}{l}660- \\
7700\end{array}$ & $\begin{array}{l}114 \times 34 \\
\times 22 \\
\text { SGSD }\end{array}$ & $\begin{array}{l}\text { Laminar/ } \\
\text { CLEARER }\end{array}$ & $\begin{array}{l}\text { The synergy angle and heat } \\
\text { transfer was minimum for } \\
\text { the largest wavy angle. }\end{array}$ \\
\hline
\end{tabular}


Table 2. (contd)

\begin{tabular}{|c|c|c|c|c|c|c|c|c|c|c|c|c|}
\hline Sr. & Author/s & & & Computati & onal dom & ain & & Thermal & $R e$ & Grid & Models/ & Remarks \\
\hline no. & and year & $\begin{array}{c}\text { Diameter } \\
\text { of the } \\
\text { tubes, } \\
D(\mathrm{~mm})\end{array}$ & $\begin{array}{c}\text { Number } \\
\text { of rows, } \\
N_{r}\end{array}$ & $\begin{array}{c}\text { Tube } \\
\text { pitch } \\
\left(S_{t}(\mathrm{~mm}) /\right. \\
\left.S_{l}(\mathrm{~mm})\right)\end{array}$ & $\begin{array}{l}\text { Type of } \\
\text { fins }\end{array}$ & $\begin{array}{l}\text { Fin length } \\
\text { or diameter } \\
(\mathrm{mm}) / \mathrm{fin} \\
\text { thickness, } \\
t_{f}(\mathrm{~mm})\end{array}$ & $\begin{array}{c}\text { Fin } \\
\text { pitch, } \\
S_{f}(\mathrm{~mm})\end{array}$ & $\begin{array}{c}\mathrm{BC}(\mathrm{Q}(\mathrm{W}), \\
\mathrm{T}(\mathrm{K}))\end{array}$ & & $\begin{array}{c}\text { size/ } \\
\text { scheme }\end{array}$ & $\begin{array}{l}\text { solution } \\
\text { algorithm }\end{array}$ & \\
\hline
\end{tabular}

$\begin{array}{ccccc}\text { 28. } \begin{array}{c}\text { Xie } \text { et al } \\ (2009)\end{array} & 16-20 & 2-7 & \begin{array}{c}32-36 / \\ 19-23\end{array} & \text { Plate }\end{array}$

(2009)

$19-23$

29. Ibrahim \& Gomaa (2009)

2-3

$\begin{array}{ccc}\text { Constant } & 660- & 114 \\ \text { temperature } & 7700 & \end{array}$
for tube,

$$
303
$$
1.5-4.5 Constant $1310-201 \times 62$ temperature 7700 for both fin and tube
Power
law

scheme
Laminar/ The effect of number of tube SIMPLE rows diminished for $N_{\mathrm{r}}>6$.

The heat transfer increased with a decrease in the tube diameter, fin spacing and with an increase in the tube pitch. The pressure drop increased with a decrease in the fin pitch and the tube diameter.

5300- $30000 \quad$ RNG- k- Four criterion for the thermal 28000, nodes, $\varepsilon /$ SIMPLEC performance were identified,

$R e_{h}$ Second $\quad$ (1) comparison of the heat order transfer and the pressure drop upwind scheme
(2) heat transfer per unit pressure drop (3) the area goodness factor (4) the efficiency index. At an angle of attack of $0^{\circ}$, all these factors were maximum. 
Table 2. (contd)

\begin{tabular}{|c|c|c|c|c|c|c|c|c|c|c|c|c|}
\hline \multirow{2}{*}{$\begin{array}{l}\text { Sr. } \\
\text { no. }\end{array}$} & \multirow{2}{*}{$\begin{array}{l}\text { Author/s } \\
\text { and year }\end{array}$} & \multicolumn{6}{|c|}{ Computational domain } & \multirow{2}{*}{$\begin{array}{c}\text { Thermal } \\
\mathrm{BC}(\mathrm{Q}(\mathrm{W}), \\
\mathrm{T}(\mathrm{K}))\end{array}$} & \multirow[t]{2}{*}{$R e$} & \multirow{2}{*}{$\begin{array}{c}\text { Grid } \\
\text { size/ } \\
\text { scheme }\end{array}$} & \multirow{2}{*}{$\begin{array}{l}\text { Models/ } \\
\text { solution } \\
\text { algorithm }\end{array}$} & \multirow[t]{2}{*}{ Remarks } \\
\hline & & $\begin{array}{l}\text { Diameter } \\
\text { of the } \\
\text { tubes, } \\
D(\mathrm{~mm})\end{array}$ & $\begin{array}{c}\text { Number } \\
\text { of rows, } \\
N_{r}\end{array}$ & $\begin{array}{c}\text { Tube } \\
\text { pitch } \\
\left(S_{t}(\mathrm{~mm}) /\right. \\
\left.S_{l}(\mathrm{~mm})\right)\end{array}$ & $\begin{array}{l}\text { Type of } \\
\text { fins }\end{array}$ & $\begin{array}{c}\text { Fin length } \\
\text { or diameter } \\
(\mathrm{mm}) / \mathrm{fin} \\
\text { thickness, } \\
t_{f}(\mathrm{~mm})\end{array}$ & $\begin{array}{c}\text { Fin } \\
\text { pitch, } \\
S_{f}(\mathrm{~mm})\end{array}$ & & & & & \\
\hline 30 . & $\begin{array}{l}\text { Tian et al } \\
\text { (2009) }\end{array}$ & 10.55 & 3 & $\begin{array}{c}21.65 / \\
25\end{array}$ & $\begin{array}{c}\text { Wavy } \\
\text { with } \\
\text { delta } \\
\text { winglet }\end{array}$ & $/ 0.2$ & 3.2 & $\begin{array}{c}\text { Constant } \\
\text { wall heat } \\
\text { flux }\end{array}$ & $\begin{array}{l}2900- \\
15356\end{array}$ & $\begin{array}{c}\text { 196, 000, } \\
\text { Hybrid } \\
\text { Scheme }\end{array}$ & $\begin{array}{c}\mathrm{k}- \\
\varepsilon / \mathrm{SIMPLE}\end{array}$ & $\begin{array}{l}\text { It was observed that the } \\
\text { delta winglet generates } \\
\text { a main vortex and a } \\
\text { corner vortex. The delta } \\
\text { winglet augmented the } \\
\text { heat transfer and pressure } \\
\text { drop. }\end{array}$ \\
\hline 31. & $\begin{array}{c}\text { Tang et al } \\
(2009 b)\end{array}$ & 18 & 6 & $34 / 42$ & $\begin{array}{l}\text { Plain } \\
\text { with } \\
\text { VGs }\end{array}$ & $/ 0.3$ & 3.1 & $\begin{array}{l}\text { Constant } \\
\text { temperature } \\
\text { for the tube }\end{array}$ & $\begin{array}{c}3870- \\
9677\end{array}$ & QUICK & $\begin{array}{c}\mathrm{k}- \\
\varepsilon / \mathrm{SIMPLEC}\end{array}$ & $\begin{array}{l}\text { Before optimization, the } \\
\text { slit fin performed better } \\
\text { than the plain fin with the } \\
\text { DWP at, but after optimizing } \\
\text { by Genetic Algorithm, the } \\
\text { fin with the DWP performed } \\
\text { better than the slit fins. }\end{array}$ \\
\hline 32. & $\begin{array}{l}\text { Zeng et al } \\
\text { (2010) }\end{array}$ & 18 & 6 & $\begin{array}{l}32-40 / \\
38-54\end{array}$ & $\begin{array}{l}\text { Plate } \\
\text { with } \\
\text { VGs }\end{array}$ & $/ 0.3$ & $2.5-4.5$ & $\begin{array}{c}\text { Constant } \\
\text { temperature } \\
\text { for the } \\
\text { inside tube } \\
\text { wall }\end{array}$ & $\begin{array}{l}5300- \\
12500\end{array}$ & $\begin{array}{c}430 \times 28 \\
\times 22 \\
\text { QUICK }\end{array}$ & $\begin{array}{c}\mathrm{k}- \\
\varepsilon / \mathrm{SIMPLEC}\end{array}$ & $\begin{array}{l}\text { The Fin pitch, transverse } \\
\text { and longitudinal tube pitch, } \\
\text { winglet length and height, } \\
\text { and winglet attack angle } \\
\text { were the six important } \\
\text { factor for the optimization } \\
\text { of the heat transfer. }\end{array}$ \\
\hline
\end{tabular}


Table 2. (contd)

\begin{tabular}{|c|c|c|c|c|c|c|c|c|c|c|c|c|}
\hline \multirow{2}{*}{$\begin{array}{l}\text { Sr. } \\
\text { no. }\end{array}$} & \multirow{2}{*}{$\begin{array}{l}\text { Author/s } \\
\text { and year }\end{array}$} & \multicolumn{6}{|c|}{ Computational domain } & \multirow{2}{*}{$\begin{array}{c}\text { Thermal } \\
\mathrm{BC}(\mathrm{Q}(\mathrm{W}), \\
\mathrm{T}(\mathrm{K}))\end{array}$} & \multirow[t]{2}{*}{$\operatorname{Re}$} & \multirow{2}{*}{$\begin{array}{c}\text { Grid } \\
\text { size/ } \\
\text { scheme }\end{array}$} & \multirow{2}{*}{$\begin{array}{l}\text { Models/ } \\
\text { solution } \\
\text { algorithm }\end{array}$} & \multirow[t]{2}{*}{ Remarks } \\
\hline & & $\begin{array}{l}\text { Diameter } \\
\text { of the } \\
\text { tubes, } \\
D(\mathrm{~mm})\end{array}$ & $\begin{array}{c}\text { Number } \\
\text { of rows, } \\
N_{r}\end{array}$ & $\begin{array}{c}\text { Tube } \\
\text { pitch } \\
\left(S_{t}(\mathrm{~mm}) /\right. \\
\left.S_{l}(\mathrm{~mm})\right)\end{array}$ & $\begin{array}{c}\text { Type of } \\
\text { fins }\end{array}$ & $\begin{array}{c}\text { Fin length } \\
\text { or diameter } \\
(\mathrm{mm}) / \mathrm{fin} \\
\text { thickness, } \\
t_{f}(\mathrm{~mm})\end{array}$ & $\begin{array}{c}\text { Fin } \\
\text { pitch, } \\
S_{f}(\mathrm{~mm})\end{array}$ & & & & & \\
\hline 33. & $\begin{array}{l}\text { Tao et al } \\
\text { (2011) }\end{array}$ & 10.55 & 2 & $\begin{array}{c}21.65 / \\
25\end{array}$ & Wavy & $\begin{array}{c}43.3 \times \\
12.5 / \\
0.05-0.42\end{array}$ & $0.5-4$ & $\begin{array}{l}\text { Constant } \\
\text { temperature } \\
\text { for tube, } \\
313 \mathrm{~K}\end{array}$ & $\begin{array}{l}500- \\
4000\end{array}$ & $\begin{array}{c}142 \times 22 \\
\times 12 \\
\text { Power } \\
\text { law } \\
\text { scheme }\end{array}$ & $\begin{array}{l}\text { Laminar/ } \\
\text { SIMPLE }\end{array}$ & $\begin{array}{l}\text { The heat transfer increased } \\
\text { with an increase in the } \\
\text { Reynolds number, the wavy } \\
\text { angle, and the fin thickness } \\
\text { and with a decrease in the } \\
\text { fin pitch and tube transverse } \\
\text { pitch. For best performance } \\
\text { a wavy angle in the range } \\
\text { from } 10^{\circ} \text { to } 20^{\circ} \text { was } \\
\text { suggested. }\end{array}$ \\
\hline 35. & $\begin{array}{l}\text { Lemouedda } \\
\text { et al (2011) }\end{array}$ & 25.4 & 3 & $\begin{array}{c}55 / \\
63.5\end{array}$ & Serrated & $\begin{array}{c}57.15 / \\
0.20\end{array}$ & 2.54 & $\begin{array}{l}\text { Constant } \\
\text { temperature } \\
(350 \mathrm{~K})\end{array}$ & $\begin{array}{l}600- \\
2600\end{array}$ & $\begin{array}{c}8100000, \\
\text { Second } \\
\text { Order } \\
\text { upwind }\end{array}$ & Laminar/ & $\begin{array}{l}\text { It was found that the serrated } \\
\text { fin gives } 9 \% \text { more heat } \\
\text { transfer as compared to the } \\
\text { plain fin with same heat } \\
\text { transfer area. However, no } \\
\text { data for pressure drop was } \\
\text { provided, therefore it is difficult } \\
\text { to assess the performance of } \\
\text { the serrated fins as compared } \\
\text { to the plain fins. }\end{array}$ \\
\hline
\end{tabular}


Table 2. (contd)

\begin{tabular}{|c|c|c|c|c|c|c|c|c|c|c|c|c|}
\hline $\begin{array}{l}\text { Sr. } \\
\text { no. }\end{array}$ & $\begin{array}{l}\text { Author/s } \\
\text { and year }\end{array}$ & \multicolumn{6}{|c|}{ Computational domain } & $\begin{array}{c}\text { Thermal } \\
\mathrm{BC}(\mathrm{Q}(\mathrm{W}), \\
\mathrm{T}(\mathrm{K}))\end{array}$ & $\operatorname{Re}$ & $\begin{array}{c}\text { Grid } \\
\text { size/ } \\
\text { scheme }\end{array}$ & $\begin{array}{l}\text { Models/ } \\
\text { solution } \\
\text { algorithm }\end{array}$ & Remarks \\
\hline 36. & $\begin{array}{l}\text { Yang et al } \\
\text { (2012) }\end{array}$ & $\begin{array}{c}219 \times 19 \\
\text { (elliptical) }\end{array}$ & & & $\begin{array}{l}\text { Wavy } \\
\text { flat }\end{array}$ & $\begin{array}{c}200 \times 19 / \\
0.26\end{array}$ & 2.30 & $\begin{array}{c}\text { Constant } \\
\text { temperature } \\
\text { without } \\
\text { conjugate } \\
\text { heat } \\
\text { transfer, } 65\end{array}$ & $\begin{array}{c}700- \\
14000\end{array}$ & $\begin{array}{c}230000- \\
253000 \text {, } \\
\text { Second } \\
\text { order } \\
\text { upwind }\end{array}$ & $\begin{array}{c}\mathrm{k}- \\
\varepsilon / \mathrm{SIMPLE}\end{array}$ & $\begin{array}{l}\text { A new type of fin was } \\
\text { proposed and its thermal- } \\
\text { hydraulic characteristics } \\
\text { were studied. }\end{array}$ \\
\hline 37. & $\begin{array}{c}\text { Banerjee et al } \\
\text { (2012) }\end{array}$ & 27 & 4 & $\begin{array}{l}36.5 / \\
21.5\end{array}$ & $\begin{array}{c}\text { Anuular } \\
\text { perforate-d }\end{array}$ & $\begin{array}{l}41 / \\
0.5\end{array}$ & 3.5 & $\begin{array}{l}\text { Constant } \\
\text { temperature }\end{array}$ & $\begin{array}{c}700- \\
14500\end{array}$ & $\begin{array}{c}100000- \\
426500\end{array}$ & $\begin{array}{c}\text { RNG- k- } \\
\varepsilon / \text { SIMPLEC }\end{array}$ & $\begin{array}{l}\text { The perforation on the back } \\
\text { side or in the wake area of } \\
\text { the fin was recommended. }\end{array}$ \\
\hline
\end{tabular}


Table 3. Summary of published correlations (heat transfer).

Sr. no. Author and year

Annular fin

1. Briggs \& Young (1963)

$N u=0.134 \operatorname{Re}^{0.681} \operatorname{Pr}^{1 / 3}\left(\frac{s}{h_{f}}\right)^{0.2}\left(\frac{s}{t_{f}}\right)^{0.1134}$

2.

Nir (1991)

Based on fin diameter: St $\operatorname{Pr}^{2 / 3}=1.0 R e_{D f}^{-0.4} W^{-0.266} R_{b}^{-0.4} K_{z, h}$

Based on tube outer diameter: St $\operatorname{Pr}^{2 / 3}=1.0 R e_{D f}^{-0.4} W^{-0.266}$

$$
R_{b}^{-0.4}\left(\frac{D_{f}}{D}\right)^{-0.4} K_{z, h}
$$

Where,

W: ratio of heat transfer area of a row of tubes to free flow area,

$K_{z, h}$ : heat transfer correction factor which depends on number of rows.

3. Watel et al (2000a, b) $N u=0.446 X R e^{0.55}$

Where $X=\left(\frac{t_{f}}{s}+1\right)^{0.55}\left(1-\frac{K}{\left(\frac{S}{D}\right)^{b}} R e^{-a}\right)^{0.55}$

$a=0.07$

For $0.034 \leq \frac{S}{D} \leq 0.14, K=0.62, b=0.27$

For $\frac{S}{D} \geq 0.14, K=0.36, b=0.55$

Valid for

$2550 \leq R e \leq 42000$

4. Pongsoi et al (2013)

$j=0.215 R e^{-0.4059}$

Crimped spiral fin

5. Nuntaphan et al (2005a) For inline: $j=3.9048 \times 10^{-4} R e^{0.0637}\left(\frac{t_{f}}{S}\right)^{-0.8363}\left(\frac{S_{l}}{S_{t}}\right)^{1.9926}$ $\left(\frac{S_{t}}{D}\right)^{2.2810}\left(\frac{D_{f}}{D}\right)^{-2.1720}$

For staggered: $j=0.1970 R e^{-0.1295}\left(\frac{t_{f}}{S}\right)^{-0.1452}\left(\frac{S_{l}}{S_{t}}\right)^{1.1874}$

$$
\left(\frac{S_{t}}{D}\right)^{0.8238}\left(\frac{D_{f}}{D}\right)^{0.0010}
$$

Valid for $600 \leq R e \leq 2700$

6. Naphon \& Wongwises

(2005)

\section{For dry conditions:}

$N u=4.0 D e^{0.464} \operatorname{Pr}^{-0.755}$ where $200 \leq D e$ (Dean number)

$\geq 3000, \operatorname{Pr}>5$.

$j=0.0178 R e^{-0.239}$ for $R e<3000$.

For wet conditions:

$N u=19.0 D e^{0.464} \operatorname{Pr}^{-0.755}$ where $200 \leq \mathrm{De} \geq 3000200 \leq D e$

$\geq 3000, \operatorname{Pr}>7$.

$j=0.029 R e^{-0.202}$ for $R e<3000$.

7. Nuntaphan et al $\quad j=0.1970 \operatorname{Re}^{m}\left(\frac{t_{f}}{S}\right)^{-2.5950}\left(\frac{S_{l}}{S_{t}}\right)^{0.7905}\left(\frac{S_{t}}{D}\right)^{0.2391}\left(\frac{D_{f}}{D}\right)^{0.2761}$

(2005b)

Where $m=-0.2871+0.5322\left(\frac{D}{S_{t}}\right)-1.2856\left(\frac{t_{f}}{S}\right)$

$+0.1845\left(\frac{S_{l}}{S_{t}}\right)$

Valid for $600 \leq R e \leq 2700$ 
Table 3. (contd)

\begin{tabular}{lll}
\hline Sr. no. & Author and year & Correlation
\end{tabular}

8. Pongsoi et al (2012a, b, c) $j=0.4132 R e^{-0.4287}$

9.

Nir (1991)

Serrated fin

Based on fin diameter: St $\operatorname{Pr}^{2 / 3}=1.0 \operatorname{Re}_{D f}^{-0.4} W^{-0.266} R_{b}^{-0.4} K_{z, h}$

Based on tube outer diameter: St $\operatorname{Pr}^{2 / 3}=1.0 \operatorname{Re}_{D}^{-0.4} W^{-0.266} R_{b}^{-0.4}$ $\left(\frac{D_{f}}{D}\right)^{-0.4} K_{z, h}$

Where,

$\mathrm{W}$ : ratio of heat transfer area of a row of tubes to free flow area,

$K_{z, h}$ : heat transfer correction factor which depends on number of rows.

10. $\quad$ Næss (2010)

$$
\begin{aligned}
N u= & 0.107 \operatorname{Re}^{0.65} \operatorname{Pr}^{1 / 3}\left(\frac{S_{t}}{D_{c}}\right)^{0.2}\left(\frac{h_{f}}{D_{c}}\right)^{-0.13}\left(\frac{h_{f}}{S}\right)^{-0.14}\left(\frac{S}{D_{c}}\right)^{-0.2} \\
& \text { for } \frac{A_{t}}{A_{\mathrm{d}}}<1 \frac{A_{t}}{A_{d}}<1 \\
N u= & 0.141 \operatorname{Re}^{0.65} \operatorname{Pr}^{1 / 3}\left(0.43+9.75 e^{\left.-3.23\left(\frac{A_{t}}{A_{d}}\right)\right)\left(\frac{h_{f}}{D_{c}}\right)^{-0.13}}\right. \\
& \left(\frac{h_{f}}{S}\right)^{-0.14}\left(\frac{h_{f}}{S}\right)^{-0.14}\left(\frac{S}{D_{c}}\right)^{-0.2} \text { for } \frac{A_{t}}{A_{d}}<1
\end{aligned}
$$

$A_{t}:$ transverse flow area, $A_{d}$ : diagnol flow area

Valid for $2500 \leq R e \leq 50000$

11. Ma et al (2012)

$N u=0.117 \operatorname{Re}^{0.717} \operatorname{Pr}^{1 / 3}\left(0.6+0.4 e^{-\frac{250 \frac{h_{f}}{S}}{R e}}\right)\left(\frac{S_{t}}{S_{l}}\right)^{0.06}$

Valid for $4000 \leq R e \leq 30000, \frac{h_{f}}{S}=5-5.5, \frac{S_{t}}{S_{l}}=0.75-1.30$

Plain fin

12. Kayansayan (1993) $j=0.15 R e^{-0.28} \in^{-0.362}$ where $=\frac{\text { total heat transfer area }}{\text { bare tube heat transfer area }}$

Valid for $500 \leq R e \leq 30000$

13. Wang et al (1996) $j=0.394 R e_{c}^{-0.392}\left(\frac{t_{f}}{D_{c}}\right)^{-0.0449} N_{r}^{-0.0897}\left(\frac{S_{f}}{D_{c}}\right)^{-0.212}$

Valid for $800 \leq R e \leq 7500$

14. Wang etal (2000) $j=0.086 \operatorname{Re}_{c}^{P 3} N_{r}^{P 4}\left(\frac{S_{f}}{D_{c}}\right)^{P 5}\left(\frac{S_{f}}{D_{h}}\right)^{P 6}\left(\frac{S_{f}}{S_{t}}\right)^{-0.93}$

where:

$P 3=-0.361-\frac{0.042 N}{\ln \left(\operatorname{Re}_{c}\right)}+0.158 \ln \left[N\left(\frac{S_{f}}{D_{t}}\right)^{0.41}\right]$

$P 4=-1.224-\frac{0.076\left(\frac{S_{l}}{D_{h}}\right)^{1.42}}{\ln \left(\operatorname{Re}_{c}\right)}$

$P 5=-0.083+\frac{0.058 N}{\ln \left(R e_{c}\right)}$

$P 6=-5.735+1.211 \ln \left(\frac{R e_{c}}{N}\right)$

$D_{h}=4 \frac{A_{c} L}{A_{o}}$

$\mathrm{L}$ is the depth of the heat exchanger along the flow direction. 
Table 3. (contd)

\section{Sr. no.}

15. (2005)

16.

Pirompugd et al

(2007a, b)

17.

Xie et al (2009)

18.

Choi et al (2010)

19.

20. $\begin{array}{cl}\text { Mirth \& } & N u=0.0197 R e_{S}{ }^{0.94}\left(\frac{S_{t}-D_{c}}{2 S}\right) \\ \text { Ramadhyani (1994) } & \begin{array}{l}\text { L is the length of the coil. } \\ \text { Valid for: } 440<R e_{\mathrm{S}}<1680 .\end{array}\end{array}$

$\begin{array}{cl}\text { Mirth \& } & N u=0.0197 R e_{S}{ }^{0.94}\left(\frac{S_{t}-D_{c}}{2 S}\right) \\ \text { Ramadhyani (1994) } & \begin{array}{l}\text { L is the length of the coil. } \\ \text { Valid for: } 440<R e_{\mathrm{S}}<1680 .\end{array}\end{array}$

Paeng et al (2010)

$N u_{\text {exp }}=0.049 R e^{0.784}$
$N u_{\text {num }}=0.097 R e^{0.671}$
Valid for: $R e=1082-1649$

$N u_{\text {exp }}=0.049 R e^{0.784}$
$N u_{\text {num }}=0.097 R e^{0.671}$
Valid for: $R e=1082-1649$.

Wavy fin

$N u=0.0197 \operatorname{Re}_{S}{ }^{0.94}\left(\frac{S_{t}-D_{c}}{2 S}\right)^{-0.3}\left\{1+\frac{111,900}{\left[\operatorname{Re}_{s}\left(\frac{L}{2 S}\right)\right]^{1.2}}\right\} \operatorname{Pr}^{1 / 3}$

$\begin{array}{cl}\text { Mirth \& } & N u=0.0197 R e_{S}{ }^{0.94}\left(\frac{S_{t}-D_{c}}{2 S}\right) \\ \text { Ramadhyani (1994) } & \begin{array}{l}\text { L is the length of the coil. } \\ \text { Valid for: } 440<R e_{\mathrm{S}}<1680 .\end{array}\end{array}$

$\begin{array}{cl}\text { Mirth \& } & N u=0.0197 R e_{s}^{0.94}\left(\frac{S_{t}-D_{c}}{2 S}\right) \\ \text { Ramadhyani (1994) } & \text { L is the length of the coil. } \\ & \text { Valid for: } 440<R e_{\mathrm{s}}<1680 .\end{array}$

\section{Correlation}

$j=1.49 R e_{c}^{0.002061 N-0.625} N_{r}^{-0.0575}\left(0.00583 N_{r}+0.825\right)$

$\varepsilon^{-0.001921 N+0.068}$

where:

$\varepsilon=\frac{\text { totalheattransferarea }}{\text { baretubeheattransferarea }}$

For $\mathrm{N}_{\mathrm{r}}=1$, (Fully wet conditions)

$j_{1, f}=0.5284\left(\frac{S}{D_{c}}\right)^{0.5440} \operatorname{Re}_{c}^{\left(0.1001 \frac{S}{D_{c}}-0.6529 \frac{S_{l}}{D_{c}}-0.06752 \frac{S_{t}}{D_{c}}-0.3734\right)}$
${ }_{\varepsilon} 0.7519$

For $\mathrm{N}_{\mathrm{r}}=1,0.65<\frac{A_{w}}{A_{o}}<1$, (Partially wet conditions)

$j_{1, p}=j_{1, f}\left(\frac{S}{D_{c}}\right)^{-1.1918} \operatorname{Re}_{c}^{\left(1.0816 \frac{S}{D_{c}}-0.06438 \frac{S_{l}}{D_{c}}-0.1133 \frac{S_{t}}{D_{c}}-0.05124\right)}$ $\left(\frac{A_{w}}{A_{o}}\right)^{-1.1861}$

For $N_{\mathrm{r}}>1$, (Fully wet conditions)

$j_{N, f}=j_{1, f} N^{0.2310}\left(\frac{S}{D_{c}}\right)^{(-0.04426 N-0.08561)}$

$\operatorname{Re}_{c}^{\left(0.02940 N-0.1308 \frac{S}{D_{c}}+0.03457 \frac{S_{l}}{D_{c}}+0.04793 \frac{P_{t}}{D_{c}}-0.1560\right)}$

$\varepsilon^{(-0.1407 N-0.08005)}$

For $N_{\mathrm{r}}>1$, (Partially wet conditions)

$j_{N, p}=j_{N, f} N^{-0.07957}\left(\frac{S}{D_{c}}\right)^{(-0.06148 N+0.07271)}$

$R e_{c}^{\left(-0.00885 N+0.06733 \frac{S}{D_{c}}+0.006928 \frac{S_{l}}{D_{c}}+0.005305 \frac{P_{t}}{D_{c}}-0.02962\right)}$

$\left(\frac{A_{w}}{A_{o}}\right)^{(0.03322 N-0.3148)}$

$N u=1.565 R e^{0.3414}\left(N \frac{S_{f}}{D}\right)^{-0.165}\left(\frac{S_{t}}{S_{l}}\right)^{-0.0558}$

Valid for : $R e=1000-6000$.

$j_{\text {inline }}=0.8692 N^{-0.0981} R e^{-0.5971}\left(\frac{S}{D}\right)^{0.0996}\left(\frac{F_{s}}{D}\right)^{-2.1720}$

$j_{\text {staggered }}=1.4534 N^{-0.0681} \operatorname{Re}^{-0.6722}\left(\frac{S}{D}\right)^{0.0652}\left(\frac{F_{s}}{D}\right)^{-0.0058}$

Valid for: $R e=500-800$.

Where $F_{\mathrm{s}}$ is the vertical fin spacing in the paper. 
Table 3. (contd)

Sr. no. Author and year

21. Wang et al (1997) $\quad j=\frac{1.201}{\left[\ln \left(R e_{s}^{\sigma}\right)\right]^{2.921}}$

Valid for: $372<\operatorname{Re}_{\mathrm{c}}<7456$.

22. Wang et al (2000) $\frac{\left(\eta h A_{o}\right)_{w}}{\left(\eta h A_{o}\right)_{\text {plain }}}=1.075\left(\frac{P_{d}}{S_{f}}\right)^{0.1425-0.03884 N}$

$P_{d}$ is the wave height here. Subscript plain is for plain fin.

Valid for: $150<\operatorname{Re}_{\mathrm{c}}<1800$.

23. Pirompugd et al $j=0.171 \varepsilon^{0.377 N} \operatorname{Re}_{c}^{(-0.0142 N-0.478)}\left(\frac{S}{D}\right)^{(0.00412 N-0.0217)}\left(\frac{A_{o}}{A_{b}}\right)^{(-0.114+0.440)}$

(2006) $\varepsilon$ is the fin factor here defined in the paper.

24. Kuvannarat et al (2006) $j=0.213262 N^{0.09891} \operatorname{Re}_{c}^{-0.51507}\left(\frac{t_{f}}{S_{l}}\right)^{0.072448}\left(\frac{A_{o}}{A_{b}}\right)^{0.600543}$

25. Pirompugd et al (2008)For $\mathrm{N}_{\mathrm{r}}=1$, (Fully wet and partially wet conditions)

$$
\begin{aligned}
& j_{1}=6.6412\left(\frac{S_{l}}{D_{c}}\right)^{-0.00085}\left(\frac{S_{t}}{D_{c}}\right)^{-2.1461} \\
& \operatorname{Re}_{c}^{\left(-0.2636 \frac{S}{D_{c}}-0.00091 \frac{S_{l}}{D_{c}}+0.1558 \frac{S_{t}}{D_{c}}-0.8865\right)}
\end{aligned}
$$

For $\mathrm{N}_{\mathrm{r}}>1$, (Fully wet conditions)

$$
\begin{aligned}
& j_{N, f}=j_{1} N^{-0.06451}\left(\frac{S}{D_{c}}\right)^{(-0.1219 N+0.7381)} \\
& \operatorname{Re}_{c}^{\left(0.03475 N+0.1145 \frac{S}{D_{c}}+0.00521 \frac{S_{l}}{D_{c}}-0.03498 \frac{P_{t}}{D_{c}}-0.04374\right)} \varepsilon^{(-0.1616 N+0.6105)} \\
& \text { For } \mathrm{N}_{\mathrm{r}}>1,(\text { Partially wet conditions) } \\
& j_{N, p}=j_{N, f} N^{-1.7838}\left(\frac{S}{D_{c}}\right)^{(-0.9459 N+3.9329)} \\
& \operatorname{Re}_{c}^{\left(-0.1554 N+1.1667 \frac{S}{D_{c}}+0.2253 \frac{S_{l}}{D_{c}}-0.1645 \frac{P_{t}}{D_{c}}+0.7158\right)} \\
& \left(\frac{A_{w}}{A_{o}}\right)^{(0.6919 N-4.7697)} \\
& \text { Valid for: } 525<\operatorname{Re}_{\mathrm{c}}<7650 . \\
& \text { Slit fin }
\end{aligned}
$$

26. Du \& Wang (2000) $j=5.98 R e_{c}^{j 1}\left(\frac{S}{D_{c}}\right)^{j 2} N_{r}^{j 3}\left(\frac{S_{w}}{S_{h}}\right)^{j 4}\left(\frac{S_{t}}{S_{l}}\right)^{-0.804}$

Where

$$
\begin{aligned}
& j 1=-0.647+\frac{0.198 N}{\ln \left(R e_{c}\right)}-0.458\left(\frac{S}{D_{c}}\right)+0.458\left(\frac{N_{r}}{R e_{c}}\right) \\
& j 2=0.116+\frac{1.125 N}{\ln \left(R e_{c}\right)}+47.6\left(\frac{N_{r}}{R e_{c}}\right) \\
& j 3=0.49+\frac{175 \frac{S}{D_{c}}}{\ln \left(R e_{c}\right)}-\frac{3.08}{\ln \left(R e_{c}\right)} \\
& j 4=-0.63+0.086 S_{n} \\
& \text { Valid for: } 200<R e_{\mathrm{c}}<8000 .
\end{aligned}
$$

Plain fins with delta winglet

$$
\text { 27. Tang et al (2009a) } \begin{aligned}
& j=43.28 R e^{-0.501}(\sin a)^{0.0143}\left(\frac{V_{h}}{V_{l}}\right)^{0.04} \\
& \text { Valid for: } 4000<R e<10000 . \\
& \text { Where } \\
& V_{h}=\text { winglet height } \\
& \\
& V_{l}=\text { winglet length } \\
& \mathrm{a}=\text { winglet attack angle }
\end{aligned}
$$


Table 4. Summary of published correlations (pressure drop).

\begin{tabular}{lcl}
\hline Sr. no. & Author and year & \\
\hline & & Annular fin \\
1. & Robinson \& & $E u=18.9 R e^{-0.316}\left(\frac{S_{t}}{D}\right)^{-0.927} N_{r}$ \\
& Briggs (1966) & Correlation \\
2. & Nir (1991) & Based on fin diameter: $f=2.12 R e_{D f}^{-0.25} W^{-0.55} K_{z, P}$ \\
& & Based on tube outer diameter: $f=2.12 R e_{D}^{-0.25} W^{-0.55}\left(\frac{D_{f}}{D}\right)^{-0.25} K_{z, P}$ \\
& Where, \\
& W: ratio of heat transfer area of a row of tubes to free flow area, \\
& $K_{z, P}:$ Pressure drop correction factor which depends on number of rows.
\end{tabular}

3. Pongsoi et al $f=0.4852 R e_{c}^{-0.2156}\left(\frac{f_{p}}{D_{c}}\right)$

(2013)

Crimped spiral fin

4. Nuntaphan et al For inline: $f=0.1635 R e^{-0.4172}\left(\frac{t_{f}}{S}\right)^{-0.5215}\left(\frac{S_{l}}{S_{t}}\right)^{-1.2235}\left(\frac{S_{t}}{D}\right)^{-0.6334}$

(2005a) $\begin{aligned} & \left(\frac{D_{f}}{D}\right)^{1.2000} \\ & \text { For staggered: } f=2.1768 R e^{-0.2679}\left(\frac{t_{f}}{S}\right)^{-0.2468}\left(\frac{S_{l}}{S_{t}}\right)^{1.8680}\left(\frac{S_{t}}{D}\right)^{0.3011} \\ & \left(\frac{D_{f}}{D}\right)^{-0.4470}\end{aligned}$

Valid for $600 \leq R e \leq 2700$

5. Nuntaphan et al $f=17.02 R e^{-0.5636}\left(\frac{t_{f}}{S}\right)^{-0.3728}\left(\frac{S_{l}}{S_{t}}\right)^{1.2804}\left(\frac{S_{t}}{D}\right)^{0.3956}\left(\frac{D_{f}}{D}\right)^{0.1738}$

(2005b) Valid for $600 \leq R e \leq 2700$

6. Pongsoi et al $f=0.3775 R e^{-0.1485}\left(\frac{S_{f}}{D}\right)^{0.4321}$

(2012a, b, c)

7. $\quad \operatorname{Nir}(1991)$

Serrated fin

Based on fin diameter: $f=1.24 R e_{D f}^{-0.25} W^{-0.32} K_{z, P}$

Based on tube outer diameter: $f=1.24 R e_{D}^{-0.25} W^{-0.32}\left(\frac{D_{f}}{D}\right)^{-0.25} K_{z, P}$

Where,

W: ratio of heat transfer area of a row of tubes to free flow area,

$K_{z, P}$ : Pressure drop correction factor which depends on number of rows.

8. Kawaguchi et al For spiral annular fin: $f=18.6 R e_{h}^{-0.228}\left(\frac{S_{f}}{t_{f}}\right)^{-0.872}$

(2004) Valid for: $600 \leq R e_{h} \leq 27000,2.95 \leq \frac{S_{f}}{t_{f}} \leq 4.39$

For spiral serrated fin: $f=6.46 R e_{h}^{-0.179}\left(\frac{S_{f}}{t_{f}}\right)^{-0.354}$

Valid for: $3000 \leq R e_{h} \leq 30000,3.07 \leq \frac{S_{f}}{t_{f}} \leq 5.07$

9. $\quad$ Næss (2010) $\quad E u=\left(0.24+\frac{8.2}{R e^{0.5}}\right) \min \left(1,0.52+964.5 e^{-3.24\left(\frac{S_{t}}{S_{l}}\right)}\right)\left(\frac{h_{f}}{D_{c}}\right)^{0.18}\left(\frac{S}{D_{c}}\right)^{-0.74}$

Valid for $2500 \leq R e \leq 50000$

10. $\quad$ Ma et al (2012) $\quad E u=1.773 R e^{-0.184}\left(\frac{h_{f}}{S}\right)^{0.556}\left(\frac{S_{t}}{D}\right)^{-0.673}\left(\frac{S_{l}}{D}\right)^{-0.133}$

Valid for $4000 \leq R e \leq 30000, \frac{h_{f}}{S}=5-5.5, \frac{S_{t}}{D}=2.3-3.2, \frac{S_{l}}{D}=2.4-3.1$.

Plain fin

11. Wang et al $f=1.039 \operatorname{Re}_{D}^{-0.418}\left(\frac{t_{f}}{D_{c}}\right)^{-0.104} N_{r}^{-0.0935}\left(\frac{S_{f}}{D_{c}}\right)^{-0.197}$

(1996)

Valid for $800 \leq R e \leq 7500$ 
Table 4. (contd)

\section{Sr. no.}

12.

Wang et al (2000)

13.

14

Mirth \& Ramadhyani

(1994)

15. Wang et al (1997)

16. Wang et al (2000)

18. Du \& Wang (2000)
17. Kuvannarat et al (2006)

\section{Wavy fin}

$$
\begin{aligned}
& F 1=-0.764+\frac{0.739 S_{t}}{S_{t}}+0.177 \frac{S_{f}}{D_{c}}-\frac{0.00758}{N_{r}} \\
& F 2=-15.689+\frac{64.021}{\ln \left(R e_{c}\right)} \\
& F 3=1.696-\frac{15.695}{\ln \left(R e_{c}\right)}
\end{aligned}
$$

Correlation

$f=20.713 R e^{-0.3489}\left(N \frac{S_{f}}{D}\right)^{-0.1676}\left(\frac{S_{t}}{S_{l}}\right)^{-0.6265}$

Valid for:

$U_{f r}=0.67-4.0 \frac{\mathrm{m}}{\mathrm{s}}, R e=1000-6000, \mathrm{D}=16-20 \mathrm{~mm}$,

$S_{f}=2-4 \mathrm{~mm}, S_{t}=38-46 \mathrm{~mm}, S_{l}=32-36 \mathrm{~mm}$

For coils 1-3 (refer to the paper): $f=\frac{8.64}{R e_{w h}^{0.457}}\left(\frac{2 S}{w h}\right)^{0.473}\left(\frac{L}{w h}\right)^{-0.545}$

For coils 3-5: $f=\frac{0.375}{R e_{w h}^{0.036}}$

Valid for: Valid for: $440<R e_{\mathrm{s}}<1680$.

$f=\frac{16.67}{\left[\ln ?\left(R_{s}\right)\right]^{2.64}}\left(\frac{A_{o}}{A_{t}}\right)^{-0.096} N^{0.098}$

Valid for: $372<R e_{\mathrm{c}}<7456$.

$\frac{\Delta P_{w}}{\Delta P_{p}}=0.64 R e_{c}^{P 1}\left(\frac{P_{d}}{S_{f}}\right)^{p 2} N^{p 3}\left(\frac{P_{d}}{D_{c}}\right)^{-0.6265} R H^{-0.06}$

Where:

$p 1=-0.18934+0.15643 \exp \left(\frac{P_{d}}{S_{f}}\right)^{2.55}\left(\frac{S_{f}}{D_{c}}\right)^{0.9}$

$p 2=-1.21912+0.07457 N^{4}\left(\frac{S_{f}}{D_{c}}\right)^{3}$

$p 3=0.65154-1.1432\left(\frac{P_{d}}{S_{f}}\right)^{3.2} \ln ?\left(R e_{c}\right)^{1.4}\left(\frac{S_{f}}{D_{c}}\right)^{1.5}$

$p 4=-0.34631\left(\frac{P_{d}}{S_{f}}\right)^{1.1}$

$f=64.0542 N^{-0.5237} R e_{c}^{-0.69284}\left(\frac{t_{f}}{S_{l}}\right)^{-0.98371}\left(\frac{A_{o}}{A_{b}}\right)^{-0.54736}$

Slit fin

$f=0.1851 \operatorname{Re}_{c}^{f 1}\left(\frac{S}{D_{c}}\right)^{f 2} N_{r}^{j-0.046}\left(\frac{S_{w}}{S_{h}}\right)^{f 3}$

Where

$f 1=-1.485+0.656 \frac{S}{D_{c}}+0.855\left(\frac{S_{t}}{S_{l}}\right)$

$f 2=-1.04-\frac{125}{R e_{c}}$

$f 3=-0.83+0.117 S_{n}$

Valid for: $200<R e_{\mathrm{c}}<8000$.

Plain fins with delta winglet

19. Tang et al (2009a)

$f=0.9856 R e^{-0.571}(\sin a)^{0.0086}\left(\frac{V_{h}}{V_{l}}\right)^{0.0014}$

Valid for: $4000<R e<10000$.

Where

$V_{h}=$ winglet height

$V_{l}=$ winglet length

$\mathrm{a}=$ winglet attack angle 
a fixed pressure drop. However, when the volume of the heat exchanger is the parameter to be considered, then the optimization of area goodness factor is not sufficient and in that case, the volume goodness factor is taken into consideration. Colburn (1942) proposed this method, which was adopted by London \& Ferguson (1949). In this method, the heat transfer coefficient $(h)$ is plotted with the normalized power requirement $\left(P_{n}\right)$ defined as

$$
\begin{aligned}
& h=\frac{\mu c_{p}}{\operatorname{Pr}^{2 / 3} D_{h}} \operatorname{Rej} \\
& P_{n}=\frac{\mu^{3}}{2 \rho^{2} D_{h}^{3}} \operatorname{Re}^{3} f
\end{aligned}
$$

A high value of $h$ vs $P_{n}$ plot indicates a more compact volume of the heat exchanger. However, this method considers a constant heat transfer coefficient throughout the depth of the heat exchanger, which is not the case in reality. Another method to determine the volume goodness factor was developed by Kays \& London (1950), which compares the heat transfer per unit heat exchanger volume and per unit temperature difference $(Z)$ with the power provided per unit heat exchanger volume $(E)$.

$$
\begin{gathered}
Z=\frac{\eta h A}{L A_{f r}} \\
E=\Delta P\left(\frac{m}{\rho_{m}}\right) \frac{1}{L A_{f r}}
\end{gathered}
$$

A high value of $Z$ with respect to $E$ indicates a more compact heat exchanger. The method developed by Kays and London has been used by most of the authors.

Therefore, along with the high heat transfer coefficient and lower pressure drop, the area goodness factor and the volume goodness factors are important in order to design a more compact and economical heat exchanger.

\section{Classification based on fin type}

In this Section, all the previous studies are divided based on the fin types considered in the present review, and these are: (A) annular fins, and (B) plate fins, and each of these are further divided into four types of fins. For annular fins, the fin types are: (1) plane annular fins (2) serrated fins, (3) crimped spiral fins, and (4) perforated fins, and for the plate fins, the fin types are: (1) plain plate fins, (2) wavy plate fins, (3) plate fins with DWP, and (4) slit and strip fins. The schematic of these fins is given in figures 2 and 3.

\subsection{Annular fins}

3.1a Plain annular fins: The annular fins are made up of rectangular plates, which are wrapped around a circular or elliptical tube (figure 2A). These fins have been used widely in the industry in the air cooling application. Briggs \& Young (1963), and Robinson \& Briggs (1966) proposed empirical correlations on the heat transfer and pressure drop characteristics of plain circular finned tubes in staggered tube arrangement and are given in table 3. Webb (1994) provided 


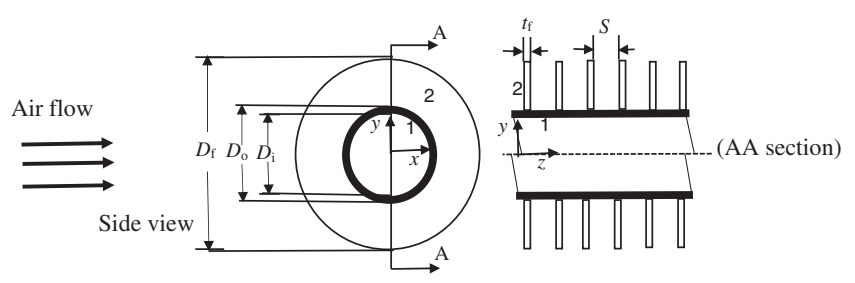

(A)

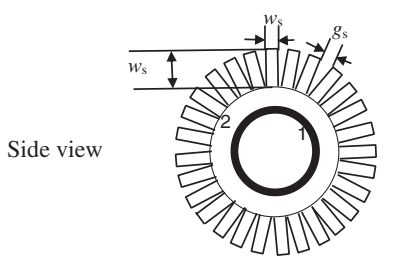

(B)

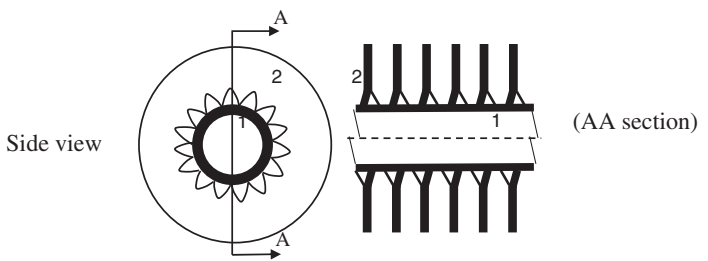

(C)

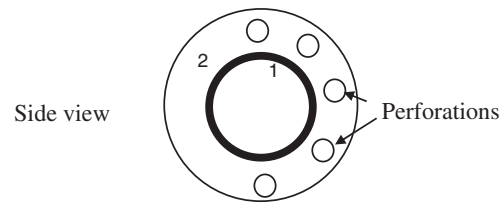

(D)

Figure 2. Schematic representation of annular fins, (A) plain annular fin, (B) serrated fin, (C) crimped spiral fin, (D) perforated fin. [(1) tube surface, (2) fin surface].

a survey of published correlations of heat transfer and pressure drop on circular finned-tubes. He concluded that all the correlations are empirical and they cannot be generalized; therefore, a single correlation could not be recommended for the practical use. Idem et al $(1990,1993)$ carried out experiments on the heat transfer and pressure drop for circular finned tubes in inline arrangement. Brauer (1964) was the first one to study elliptical finned tubes in dry conditions. Only few experimental studies were carried out on elliptical tubes in dry conditions prior to 1990s.

The Correlations available in the literature prior to 1990s for annular finned tubes were based on the assumption of uniform convective heat transfer coefficient on the fin surface, and the local flow phenomenon was not understood. Hu \& Jacobi (1993) performed experiments on 1-row annular-finned tube using the naphthalene sublimation technique to understand the flow physics. The experiment was setup in a wind tunnel with a blower near the outlet, and the depth gauge was used to record the contour measurements at 360 locations on the fin. Sh distribution on the fin surface was measured, and then, the heat and mass transfer analogy was used to obtain the heat transfer and the fin efficiency. It was observed that, at low Reynolds number, the Sherwood 


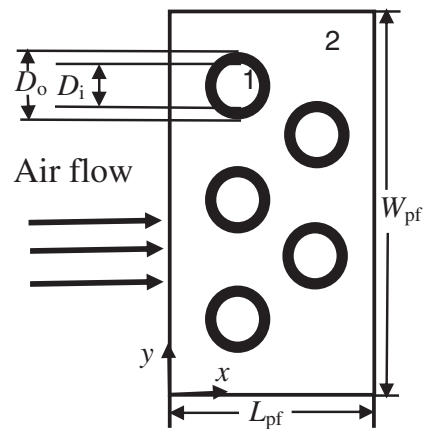

Side view Front view

(A)

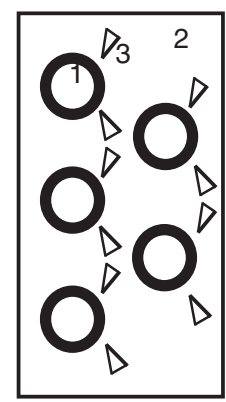

Side view
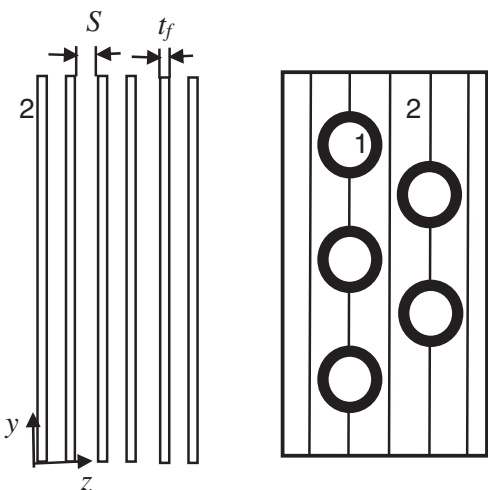

Side view

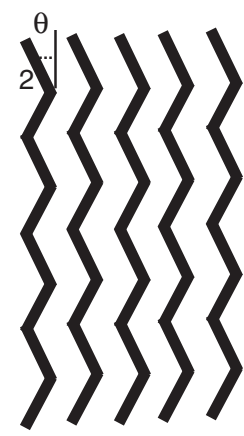

Front view

(B)

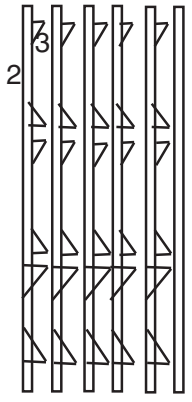

Front view

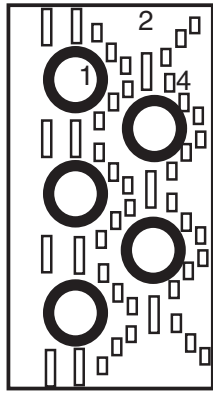

Side view

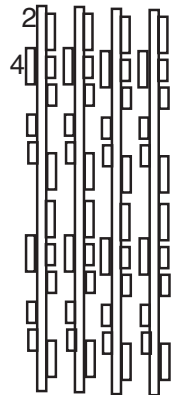

Front view

(C)

(D)

Figure 3. Schematic representation of plate fins, (A) plain plate fin, $(\mathbf{B})$ wavy plate fin, $(\mathbf{C})$ plate fin with delta winglet, (D) plate fin with slits. [(1) tube surface, (2) fin surface, (3) delta winglets, (4) slits].

number was high near the fin tip and fin root only, and as the Reynolds was increased beyond $3.7 \times 10^{4}$, the Sherwood number increased over the whole surface of the fin. The periodic peaks in the mass transfer were observed on the fin and the location of the peaks was found to be little dependent on the Reynolds number, and these peaks were compared with the Crow instability (1970). The radially averaged Sherwood number was higher in front of the tube and lower in the wake region as expected. The fin efficiency was found to be a function of the fin parameters (fin diameter, thermal conductivity and heat transfer coefficient) and the fin efficiency of aluminum was found to be larger than the fin efficiency of mild steel.

Jang \& Yang (1998) performed experimental as well as numerical studies in both the dry and wet conditions for the annular-finned tubes. The aim was to study the heat transfer and pressure drop characteristics of elliptical tubes $\left(a_{1} \times b_{1}=36 \mathrm{~mm} \times 12.7 \mathrm{~mm}\right)$ and compare it with the circular tubes $(O D=12.7 \mathrm{~mm})$ in wet and dry conditions. It was observed that the Colburn factor and the friction factor decrease with an increase in the Reynolds number. The heat transfer coefficient was found to be larger for the wet coils as compared to the dry coils for the circular as well as for the elliptical tubes. The heat transfer coefficient was lower 
by $30-35 \%$, and the pressure drop was lower by $25-30 \%$ for the elliptical tubes as compared to the circular tubes. The numerical $\mathrm{Nu}$ overestimated the experimental $\mathrm{Nu}$ by $35 \%$ due to the constant temperature boundary condition on the fin; however, numerical pressure drop satisfied the experimental pressure drop quite well. The heat transfer per unit pressure drop was 50\% higher for the elliptical tubes as compared to the circular tubes.

In the studies of Hu \& Jacobi (1993) and Jang \& Yang (1998), the effect of fin spacing and other parameters was not investigated. This motivated Watel et al (1999) to perform the experiments to study the effect of fin spacing and Reynolds number on the $\mathrm{Nu}$ of the annular finned tube. The experiments were performed in a wind tunnel of dimensions $400 \mathrm{~mm} \times 300 \mathrm{~mm}$. A radiant panel with infrared waves was used as the heating source for the fins to obtain a temperature range of $110^{\circ} \mathrm{C}$ to $140^{\circ} \mathrm{C}$. The tube diameter was $58 \mathrm{~mm}$, the fin diameter was $100 \mathrm{~mm}$, and the fin spacing was varied from 2 to $40 \mathrm{~mm}$. The flow visualization was performed with the help of the PIV, and it was observed that the rotation of the fin does not affect the $\mathrm{Nu}$ up to a value of rotational $R e=2500$ (based on the rotational velocity of fin). However, for rotational $R e>2500$, the flow pattern got affected by the rotation of the fin. The $N u$ increased with an increase in the fin spacing, because at larger fin spacing, there was no interaction between the boundary layers developed at two fin surfaces. Also, $N u$ increased with an increase in the rotational $R e$ due to the thinning of the boundary layer at higher rotational $R e$. One correlation for $\mathrm{Nu}$ was obtained for various fin spacing and Reynolds numbers. After studying the effect of the fin spacing on the convective heat transfer, Watel et al (2000a, b) performed experiments to study the effect of the rotational speed of the annular fins on the convective heat transfer of the fins, and the importance of the natural convection in this process. The experiments were performed in the similar manner and it was found that, at low rotational speed, $N u$ was independent of the rotational speed, and the heat transfer was mainly by the natural convection and as the rotational speed increased, the forced convection dominated over the natural convection. $N u$ increased with an increase in the fin spacing for both the natural and the forced convection. After performing experiments to determine the importance of the natural convection for a rotating fin, Watel et al (2000b) performed experiments to study the heat transfer from a rotating annular fin subjected to an external air flow and concentrated only on the forced convection part. For a rotational $900 \leq R e \leq 9000$, the effect of the rotation started influencing the flow pattern, and for a rotational $R e=1.8 \times 10^{4}$, the air flow pattern was similar to the case where external air flow was not present.

As discussed earlier, in this section that the heat transfer predicted by the numerical study performed by Jang \& Yang (1998) overestimated their experimental heat transfer because of the negligence of the fin efficiency. To incorporate the fin efficiency, Mon \& Gross (2004) performed numerical study to investigate the thermal-hydraulic performance of the annular-finned tubes for a range of $8.6 \times 10^{3} \leq R e \leq 4.3 \times 10^{4}$. The numerical domain is shown in figure 4 (similar type of computational domain has been used by other authors as well). They observed that the hydrodynamic boundary layer starts developing from the leading edge of the fin, and reaches a maximum thickness at the fin-tube junction. The horseshoe vortices formed at the fin-tube junction, and their formation was more dominant for larger fin spacing. The heat transfer coefficient was found to be lower for the inline arrangement as compared to the staggered arrangement. For staggered arrangement, the heat transfer coefficient increased with the fin spacing up to $S=2$ $\mathrm{mm}$, and after that it became constant, but for inline arrangement, the heat transfer coefficient kept on increasing with the fin spacing. However, the pressure drop decreased with an increase in the fin spacing for both the arrangements.

Chen \& Hsu (2008) developed a finite difference method in conjunction with the least square method and the temperature measurements to predict the temperature distribution and the local 


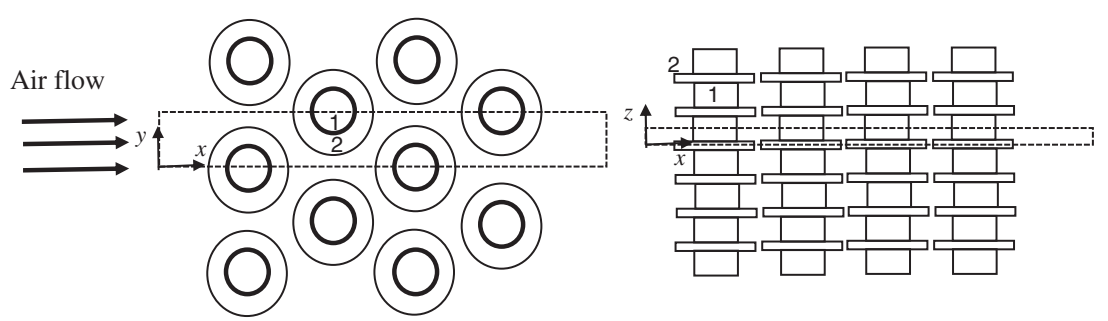

Figure 4. Computational domain of (Mon \& Gross 2004).

heat transfer coefficient on the fin surface. The experiments were performed in a $550 \times 450 \times$ $350 \mathrm{~mm}$ box with a finned tube $\left(O D=27 \mathrm{~mm}, D_{f}=99 \mathrm{~mm}\right)$. It was observed that the heat transfer coefficient was highest on the bottom part of the fin as compared to the top portion of the fin (approximately 9 times). The heat transfer coefficient increased and the fin efficiency $(\eta)$ decreased with the fin spacing; however, both reached an asymptotic value as $S \rightarrow \infty$. The results were compared with the correlation from the book of Raithby \& Hollands (1985) and Kreith \& Bohn (1993) and were found in good agreement.

Pongsoi et al (2013) performed experiments to study the air side performance of the L-footed spiral annular fins. The effect of the fin spacing was studied on the air side performance of the heat exchangers. The experimental setup was the same as used by (Wongwises \& Chokeman 2005) (see Section 3.2b). The average heat transfer rate and the average pressure drop increased with a decrease in the fin spacing. The Colburn factor was found to be independent of the fin spacing at all Reynolds number, however, the friction factor increased as the fin pitch was increased for $R e>6000$, and below $R e<6000$, the fin pitch did not affect the friction factor. The correlations for the Colburn factor and the friction factor were proposed and are given in table 3.

3.1b Serrated fins: The serrated fins are made up by cutting the tip of the plain annular fins into many segments. Figure $2 \mathrm{~B}$ shows the schematic of the serrated fins, the front view (AA section) of the serrated fin is similar to the front view of the plain annular fin and is not given. Further, only segment parameters are shown in figure 2B (fin-tube parameters are similar to that of the plain annular fin). The boundary layer breakup by these segments is the attractive feature of the serrated fins, which enhance mixing and heat transfer, however, it gives rise to more pressure drop as well. Previous studies on the serrated fins focus mostly on its heat transfer per unit pressure drop performance as compared to the plain annular fin. Nir (1991) argued that the published correlation for the heat transfer and pressure drop characteristics of the finned-tube banks have been formulated based only on the data, and the understanding of the flow patterns has not been considered. Therefore, they tried to modify the previous correlations based on the understanding of the flow patterns and proposed new correlations for the plain and segmented annular fins. Hashizume et al (2002) carried out analytical and experimental investigation to study the fin efficiency of the serrated fins. The analytical model was developed based on two assumptions: (1) uniform heat transfer coefficient on the fin surface, and (2) no heat transfer from the tip of the fins. Fin efficiency based on these assumptions was named as the theoretical fin efficiency. To account for these two assumptions and to obtain a real fin efficiency, a correction factor was introduced, which was calculated experimentally. The fin materials were copper, carbon steel and stainless steel. The fin efficiency calculated with the analytical model after incorporating the correction factor was in good agreement with the experimentally measured fin efficiency. The 
correlation for the fin efficiency was proposed. The comparison between the spiral annular and serrated fins was first presented by Kawaguchi et al (2004). In the two consecutive studies, they investigated the heat transfer and pressure drop characteristics of the spiral annular fins and serrated fins with varying fin pitch, tube pitch and row number. The experiment was setup in a wind tunnel with air blower and water circulating supply. For a fin pitch of $5 \mathrm{~mm}$, for the serrated fins, the friction factor was found to be 1.15 times the friction factor for the spiral fins. The turbulence generated by the serrated fins was believed to be the reason for this increase in the friction factor. However, smaller force requirement for changing the flow direction across the fins for serrated fins, and entrainment of the flow in the wake region due to turbulence generated by segments were the two factors, which limited the friction factor for the serrated fins. For a fin pitch of $3.3 \mathrm{~mm}$, the friction factor for the serrated fins was 1.1 times the friction factor for the spiral fins. It was observed that the friction factor increases with a decrease in the fin pitch for both types of fins; however, the increase was larger for the plain spiral fins. The effect of transverse and longitudinal tube pitch was found to be insignificant on the friction factor. It was observed that the pressure drop increases with an increase in the row number from 3 to 6 , but the increase in the friction factor was insignificant. The effect of fin pitch on the heat transfer was larger for the plain spiral fins as compared to the serrated fins. The heat transfer coefficient per unit pressure drop was highest for the serrated fins for a fin pitch of $5 \mathrm{~mm}$ among all the combinations. Næss (2010) performed experiments on the serrated-finned-tube heat exchangers to study the effect of the tube bundle layout, the tube and the fin geometry. The experiments were performed in a wind tunnel with hot air flow $\left(100^{\circ} \mathrm{C}\right)$ using a blower with cold water on the tube side. Ten samples of heat exchangers were investigated in the study. It was found that $N u$ shows a maximum when the flow areas in the transverse and diagonal directions become equal. Increasing the fin spacing resulted in a decrease in the heat transfer coefficient, whereas the heat transfer coefficient increased with an increase in the fin height. The pressure drop decreased with an increase in the fin spacing, whereas the effect of fin height on the pressure drop was found to be negligible.

Martinez et al (2010) performed experiments to study the heat transfer and pressure drop characteristics of helically segmented-finned-tube heat exchangers and compared the results with the correlations of Weierman (1976), Weierman et al (1978), Nir (1991), Ganapathy (2003) and Kawaguchi et al (2004). The results were compared in terms of the pressure drop, overall heat transfer coefficient, flue gas outlet temperature and water outlet temperature. The heat exchanger was placed in a rectangular duct with flue gas coming from a chimney with dampers to control the flow rate. The inlet gas temperature was varied from $179^{\circ} \mathrm{C}$ to $184^{\circ} \mathrm{C}$ and $T_{\text {wi }}$ was varied from $105^{\circ} \mathrm{C}$ to $128^{\circ} \mathrm{C}$. The pressure drop obtained from the correlation of Weierman satisfied the experimental data within $89 \%$ and Nir's correlation satisfied the experimental data within $65 \%$. Despite the error, both the correlations showed a higher the pressure drop than the experimental pressure drop and hence were recommended for the design purpose. Similarly the results for heat transfer, flue gas outlet temperature and water outlet temperature were compared. Overall, the correlation of Kawaguchi et al (2004) was recommended for the heat transfer design of the compact heat exchanger. In the cases where the finned tube geometry and fluid conditions were outside the range of Kawaguchi's correlations, the Weierman's correlation was recommended.

The experimental investigations on the serrated fins give its thermal-hydraulic performance, however to obtain the 3D flow pattern, 3D numerical simulations are required. For this purpose, Lemouedda et al (2011) performed numerical investigation to study the heat transfer performance of the serrated fins and compared it with the plain fins. The numerical model consisted of the helical configuration of the serrated fins with two rotations. Based on their Reynolds number range (600-2600), they assumed the flow to be in the laminar region and applied a laminar model. To study the effect of fin serration, the heat transfer from the serrated fin was compared 
with the plain fin with equivalent heat transfer area (less fin height) and with plain fin with the same height (more heat transfer area). It was found that the serrated fin gives $9 \%$ more heat transfer as compared to the plain fin with the same heat transfer area. However, with the same height the plain fin and serrated fin performed equally well. Further, the segments were twisted from $0^{\circ}$ to $25^{\circ}$ and it was found that the heat transfer performance increases up to an angle of $15^{\circ}$ and then it decreases. In this study, no data for the pressure drop performance was provided; therefore, it is difficult to assess the performance of the serrated fins as compared to the plain fins based on this numerical study.

Ma et al (2012) carried out experimental investigation to study the effect of fin spacing, fin height, tube pitches on the heat transfer and the pressure drop characteristics of the serrated finned tubes. The experiments were performed in a closed wind tunnel consisting of a hot air loop to heat the cold water coming from the water tank. The heat transfer decreased in the range from 11 to $0 \%$ as the $h_{\mathrm{f}} / S$ ratio increased from 5 to 5.5 for a range of $4000 \leq R e \leq 30000$. A critical $R e$ was found, above which the effect of the $h_{\mathrm{f}} / S$ ratio on the heat transfer was negligible. The transverse tube pitch had negligible effects on the heat transfer, whereas the heat transfer was affected by the longitudinal tube pitch and it was concluded that there should be an optimum $S_{\mathrm{t}} / S_{1}$ ratio for a specific transverse tube pitch. The Euler number increased by $8 \%$, when the $h_{\mathrm{f}} / S$ ratio increased from 5 to 5.5 and it decreased by $20 \%$ with an increase in the transverse tube pitch from $88 \mathrm{~mm}$ to $120 \mathrm{~mm}$, whereas the effect of longitudinal pitch on the Euler number was negligible. The correlation was given for $\mathrm{Nu}$ and $\mathrm{Eu}$.

3.1c Crimped spiral fins: The crimped spiral fins are shown in figure $2 \mathrm{C}$, and the geometrical parameters are the same as shown for the plain annular fin and are not shown. These fins are designed to produce more disturbance in the air flow as compared to the plain annular fins, which should enhance the turbulence and mixing in the flow and hence is expected to enhance the heat transfer. However, the design of the fin must be optimized to minimize the increase in the pressure loss due to more resistance to the flow. In the literature prior to 2004, only plane annular fins were studied and the first study on crimped spiral fins was performed by Nuntaphan et al (2005a). They investigated the effects of fin spacing, tube diameter, and fin height and tube arrangement on the heat transfer and pressure drop of the exchanger. Twenty-three samples of heat exchangers were tested with varying tube diameters (17 $\mathrm{mm} \leq D \leq 27 \mathrm{~mm})$, fin spacing (2 $\mathrm{mm} \leq S \leq 7 \mathrm{~mm})$, fin height $\left(10 \leq h_{\mathrm{f}} \leq 15 \mathrm{~mm}\right)$ transverse $\left(50 \mathrm{~mm} \leq S_{\mathrm{t}} \leq 84 \mathrm{~mm}\right)$ and longitudinal pitch $\left(24.2 \mathrm{~mm} \leq S_{\mathrm{t}} \leq 50 \mathrm{~mm}\right)$ in the inline and staggered arrangement. For both inline and staggered arrangements, it was observed that the pressure drop increases and the heat transfer coefficient decreases with an increase in the tube diameter. The reason was believed to be the ineffective area behind the tubes as explained by Wang et al (2002). The heat transfer coefficient decreased with an increase in the fin height for both the arrangements; however, the pressure drop was independent of the fin height for the staggered arrangement, whereas it increased with an increase in the fin height for the inline arrangement. Correlations for the Colburn factor and the friction factor were proposed for the inline and staggered arrangements. In a similar manner, Nuntaphan et al (2005b) investigated 10 samples of crimped spiral-finned-tube heat exchangers in the dehumidifying conditions. It was found that the heat transfer coefficient for a wet surface was lower as compared to the dry surface for $R e<2000$. One possible reason was believed to be the water film resistance and the condensate blocking. However, at higher Reynolds number, the heat transfer coefficient for the wet surface was found to be slightly higher than the heat transfer coefficient for the dry surface. The effect of fin height was not found to be as dominant as the effect of the condensate resistance. At smaller fin spacing, the heat transfer 
rate was lower, this was due to the enhanced resistance offered by the condensate, therefore leading to more airflow bypass. The effect of the wet conditions on the pressure drop was small for large fin spacing, but it was significant for smaller fin spacing.

Naphon \& Wongwises (2005) presented the inside and outside heat transfer coefficients for the spirally coiled finned tube heat exchanger under dry and wet surface conditions. The experiments were performed in an open wind tunnel with blowers and flow straightener. The mathematical model was also developed to predict the thermal performance of the heat exchanger. The heat exchangers consisted of six layers of spiral coils with four turns each layer and the innermost circle and outermost circle were of diameter 145 and $350.4 \mathrm{~mm}$. $T_{\text {ao }}$ was found to increase with an increase in the air mass flow rate, but beyond $0.1 \mathrm{~kg} / \mathrm{s}$, the effect was negligible. For the wet conditions, $T_{\text {wo }}$ increased with an increase in the water mass flow rate. For the dry conditions, $T_{\text {wo }}$ decreased slightly further with the water mass flow rate, and the effect of $T_{\mathrm{ai}}$ on $T_{\mathrm{wo}}$ was insignificant. The heat transfer coefficient increased rapidly with an increase in the air mass flow rate but was unaffected by the $T_{\text {ai }}$. Correlations were proposed for $\mathrm{Nu}$ and the Colburn factor for the dry and wet surface conditions. Wongwises and Naphon (2006a, b) extended their study on the spirally coiled-finned-tube heat exchangers and performed experiments under wet-surface conditions. The experimental setup and the mathematical model was similar to the previous study. The mathematical model overpredicted the experimental results by small fraction, and the reason behind the discrepancy was believed to be the negligence of the water film resistance in the mathematical model. The enthalpy effectiveness and humidity effectiveness were defined and their behavior was also described. The liquid film had a significant effect on the heat transfer of the heat exchanger. Similar to the study in the wet surface conditions Wongwises \& Naphon (2006b) performed experiments in the dry surface condition. The experimental setup and the other parameters were the same. In dry conditions, the agreement between the mathematical model and the experiments was better as compared to the wet surface conditions due to the absence of the moisture in the air. The effect of the air mass flow rate was significant on the heat exchanger effectiveness and heat transfer, whereas the effect of the water mass flow rate on these quantities was negligible.

Earlier in this section, we have discussed the study performed by Nuntaphan et al (2005a) on the effect of fin pitch on the air side heat transfer coefficient for spiral-finned-tube heat exchanger. However, the frontal velocity was in the range of $0.5 \leq U_{f r} \leq 1.5 \mathrm{~m} / \mathrm{s}$, which has been considered to be low for the commercial applications. This motivated Pongsoi et al (2011) to perform the experimental study to investigate the effect of fin pitch, Reynolds number, fin material and number of tube rows on the air side performance of the spiral-finned-tube heat exchanger for a frontal velocity range of $2 \leq U_{f r} \leq 6 \mathrm{~m} / \mathrm{s}$. The experimental setup was the same as used by Naphon \& Wongwises (2005). They argued that the effect of fin pitch was very small on the Colburn factor due to high value of $R e$ in their study, which promotes better mixing, also, for one or two rows, it was found that the boundary layer does not develop, and this was considered to be the second reason behind this. However, this observation is not in agreement with the other studies, which we have discussed so far. The effect of fin pitch on the friction factor was insignificant for $3.2 \mathrm{~mm} \leq f_{p} \leq 4.2 \mathrm{~mm}$; however, the friction factor increased for $f_{p}=6.2 \mathrm{~mm}$. The copper fin showed a little higher fin efficiency than the aluminum fins. In the continuation of their previous study, Pongsoi et al (2012a, b, c) studied the effect of number of tube rows on the performance of the crimped spiral-finned-tube heat exchanger with multipass semi-parallel-andcounter current flow configuration. The experimental setup was the same as used in the previous study. It was found that the number of tube rows does not affect the Colburn factor and the friction factor beyond a $R e>2000$. The reason was believed to be the shedding of the downstream turbulence eddies, which causes good mixing. Both the average heat transfer and pressure drop 
increased with an increase in the number of tube rows. The correlations for the Colburn factor and the friction factor were presented in the study. In the similar study on the same experimental setup Pongsoi et al (2012a) studied the effect of number of tube rows along with the variation in the fin height. The effect of the number of tube rows was similar as were found in the previous study. The only difference in the results was the decrease in the pressure drop with an increase in the fin diameter. After performing the studies on the effect of the fin spacing, number of tube rows and fin height on the air side heat transfer, Pongsoi et al (2012b) tried to optimize the heat exchanger with respect to the fin spacing for a crimped-spiral-finned-tube heat exchanger. The experimental setup was similar to the previous study. Three types of heat exchanger indexes were defined in the study. First one was the ratio of the heat transferred to the air side to the pressure drop of heat exchanger, and was called the heat exchanger performance index as it did not include the other components than the heat exchanger. The second performance index was the system performance index, which included the pumping fan performance along with the performance of the heat exchanger. It was defined as the ratio of the average heat transfer rate to the pressure drop at the operating point of heat exchanger (operating point was obtained from the $P-Q$ curve of fans and pressure drop of heat exchanger with different fin spacing). The third one was the dimensionless system performance index, which was obtained by modifying the second performance index, and it was defined as the ratio of the desired heat transfer rate to the provided pumping power. The effect of the fin spacing on the air side heat transfer performance was similar as described in their previous study (Pongsoi et al 2011). Based on the three performance indexes, an optimum fin spacing of $4.2 \mathrm{~mm}$ was found to be the best.

3.1d Perforated fins: The plain annular fin has been investigated by many researchers in the past as we have discussed in Section 3.1a. Banerjee et al (2012) modified the plain annular fins with perforations on the fin (figure 2D). The heat transfer and pressure drop characteristics were studied with different locations of the perforations using numerical simulations. The effect of the non-uniform fin spacing on the pressure drop was also studied. One computational domain consisted of the four rows of the tubes to study the perforated fins, and another computational domain was the 2D geometry of the half symmetry of the A type air cooled condenser to study the effect of non-uniform fin spacing. The frontal air velocity was varied from 1 to $5 \mathrm{~m} / \mathrm{s}$ and the corresponding range of Reynolds number was $4000<R e<24000$. The performance of the perforated fins was found to be dependent on the location of the perforation on the fin and the perforations on the back side (in the wake area) of the fin were recommended. The $Q / \Delta P$ ratio was found to be 1.23 and 1.05 for two particular perforation locations with respect to the solid fins. The non-uniform fin spacing was also found to be beneficial and for two different cases, and the pressure drop was found be lower than the uniform fin spacing case.

\subsection{Plate fins}

3.2a Plain plate fins: Plain plate fins are used extensively in the air-cooled heat exchangers (figure 3A) (now onwards in the paper plain plate fin will be referred as plain fin). Rich (1973) provided a survey of the published work relating to the heat transfer of multi-row plate finnedtube heat exchangers in the form of average heat transfer coefficients. The quantitative data on the heat transfer for 2-rows plate-finned-tube heat exchanger was not known prior to 1975. This motivated Saboya \& Sparrow (1976) to apply the heat and mass transfer analogy in conjunction with the naphthalene sublimation technique to investigate the heat transfer for a two row plate-finned-tube heat exchanger for a range of $211 \leq R e_{\mathrm{h}} \leq 1089$. The fins were made up of 
naphthalene and the tubes between the fins were made up of delrin (plastic). The finned tubes were placed in a channel along with a rotameter, gas meter, and a blower. The heat transfer coefficient was found to be dependent on two parameters, one was the boundary layer development and the other was the vortex generation. For the first row, the mass transfer coefficient was largely affected by the boundary layer development, and for the second row, the mass transfer coefficient was mainly dependent on the vortex generation. At low Reynolds number, the effect of the boundary layer was more as compared to the vortex generation; however, at higher $R e$, the effect was reversed. Overall, the mass transfer coefficient was higher for the first row as compared to the second row. Rich $(1973,1975)$ examined the effects of fin spacing, and number of tube rows $\left(2 \leq N_{\mathrm{r}} \leq 6\right)$ for heat exchangers. Elmahdy \& Biggs (1979) determined the $m$ exponent of Reynolds number for $200 \leq R e \leq 2000$. McQuiston (1981) developed a simple correlation for the Colburn factor for four row staggered bank of plain fins for $100 \leq R e \leq 4000$. In all these previous studies, the range of the Reynolds number was very small. Therefore, to consider a larger range of Reynolds number $(100 \leq R e \leq 30000)$, Kayansayan (1993) performed experiments with 10 samples of heat exchangers. He studied the effect of outer geometry $(9.52 \mathrm{~mm}$ $\leq D \leq 16.3 \mathrm{~mm}, 2.2 \mathrm{~mm} \leq S \leq 4.2 \mathrm{~mm}, 12 \leq N_{\mathrm{r}} \leq 19, S_{\mathrm{t}}=22 \mathrm{~mm}, S_{1}=40 \mathrm{~mm}$ ) upon the performance of plate-fin-tube heat exchanger. However, the channel effect of the fins was neglected in the study. The Colburn factor was presented with respect to the varying Finning factor (ratio of total heat transfer area to the tube outside area) and the Colburn factor decreased as the Reynolds number was increased. A correlation by regression analysis was obtained for the Colburn factor. Rocha et al (1997) performed 2D numerical simulations to study 1-row and 2-row circular and elliptical plate-finned-tube heat exchangers for range of $299 \leq R e_{h} \leq 1576$. The fin efficiency based on the average $N u$ was obtained, and was compared with the fin efficiency obtained by Rosman et al (1984), and it showed good agreement. It was found that the elliptical arrangement with $e=0.5$ and two rows of tubes was the most efficient arrangement, and the efficiency of the elliptical arrangement was $18 \%$ higher than the circular arrangement.

In HVAC\&R applications, the use of smaller diameter tubes has been very popular. Wang et al (1996) focused their study on the smaller diameter tubes $(D=9.52 \mathrm{~mm})$ and carried out experiments in an open wind tunnel with 15 samples of heat exchangers. It was observed that the Colburn factor and the friction factor decrease with an increase in the Reynolds number, and the Colburn factor showed a maximum for $R e<2000$ for two samples $\left(f_{p}=1.75 \mathrm{~mm}\right.$ and $1.85 \mathrm{~mm}, N_{r}=6$ ). It was believed that the increase in the size of the standing vortices behind the tubes at larger number of rows and reduction in the vortex size (wake region) at smaller fin pitch resulted in the maxima in the Colburn factor. For $S=1.57 \mathrm{~mm}$, the Colburn factor decreased with an increase in the number of tube rows for $R e<2000$, and for $R e>2000$, the effect of tube rows diminished. The effect of fin spacing on the Colburn factor and the friction factor was negligible. Correlations were developed for the Colburn factor and the friction factor. Wang \& Chi (2000) and Wang et al (2000) carried forward the study on smaller diameter tubes (7 $\mathrm{mm} \leq D \leq 9.52 \mathrm{~mm})$ and investigated 18 samples of heat exchangers $\left(1.22 \mathrm{~mm} \leq f_{p} \leq\right.$ $\left.2.3 \mathrm{~mm}, 2 \leq N_{r} \leq 4\right)$. The effect of fin pitch on the Colburn factor was negligible for $N_{r}>4$ and $R e>2000$. The heat transfer was found to increase with a decrease in the fin pitch for $300 \leq R e \leq 3000$ and $N_{r}=1,2$. At low fin pitch, the Colburn factor decreased with an increase in the number of tube rows for $R e<3000$, and for $R e>3000$, there was no significant effect of tube rows on the Colburn factor. The Colburn factor was found to be higher and the friction factor was found to be lower for the smaller diameter tubes, and the correlations for the Colburn factor and the friction factor were proposed.

The effect of different geometrical parameters on the heat transfer performance of the aircooled heat exchangers has been studied by many researchers as we have discussed in previous 
sections, however, in some studies, the results contradict. For example in similar studies, Rich (1973) and Wang et al (1996) argued that the effect of fin pitch was negligible for plainfinned-tube heat exchangers. However when the data of Wang et al (1996), Kayansayan (1993), McQuiston (1978b), and Seshimo \& Fuji (1991) was plotted together by Wang et al (2000b), then the effect of the fin spacing could be clearly seen on the Colburn factor. To study the deviation in the results, Wang et al (2000b) carried out a theoretical study on the data reduction method for air-cooled heat exchangers.

The main conclusions of the study were:

(1) The energy balance in the experiments for the air side and the tube side should be less than $5 \%$, and for better accuracy for the water side heat transfer rate, the temperature drop on the water side should be larger than $2^{\circ} \mathrm{C}$.

(2) $\varepsilon$-NTU correlation must be used carefully for the data reduction according to the circuitry design.

Prior to 2002, different types of experiments were performed to measure the heat transfer coefficients on the air side and for the temperature measurements, most of the researchers had used thermocouples. Ay et al (2002) introduced the use of Infrared thermography to determine the heat transfer coefficient for the plate-finned-tube heat exchangers in inline and staggered arrangement of tube. Infrared thermography detects the infrared radiation emitted by the objects when the temperature of the object is above the absolute zero and the amount of radiation emitted increases with an increase in the temperature. Along with the infrared thermography to record the temperature distribution, a control volume based finite difference method was also developed for the numerical calculations. It was found that at the leading edge, the heat transfer coefficient was higher because the thermal boundary layer just started to develop. There was a wake formation behind the tubes, and the wake region was smaller for the staggered arrangement as compared to the inline arrangement. The heat transfer coefficient was found to decrease towards the outlet edge of the fin at low Reynolds number; however, it shows a different behavior at high Reynolds number due to the presence of the turbulence. The heat transfer coefficient was found to be larger for the staggered arrangement as compared to the inline arrangement.

Matos et al (2004a) carried out numerical simulations and experimental investigation to optimize the staggered circular and elliptical finned tubes in a fixed volume. The simulations were performed for the non-finned tubes [similar to Matos et al (2001)] and was validated with the experiments. The experiments were performed in a wind tunnel with a test section of dimensions $0.171 \mathrm{~m} \times 0.161 \mathrm{~m} \times 0.152 \mathrm{~m}$. The effect of three parameters (1) tube pitch, (2) tube eccentricity, and (3) fin spacing, on the heat transfer and pressure drop and finally on the optimization of the heat exchanger was studied. The numerical results showed a good qualitative agreement with the experimental results; however, the small quantitative errors were observed and the reason for that was assumed to be the constant temperature boundary condition in the numerical simulations, and constant heat flux condition in the experiments. The dimensionless thermal conductance $\left(q^{\prime}\right)$ showed a maxima for a particular dimensionless tube spacing $\left(S_{t} / D=0.25\right)$ and tube eccentricity (0.5). Overall, the heat transfer was found to be higher for the elliptical tube for a fixed pressure drop condition. In the similar manner, (Matos et al 2004b) carried out numerical simulations and experimental investigation to optimize the staggered circular and elliptical finned tubes in a fixed volume. The results obtained were the same as given in Matos et al (2004b), however, the optimization of the third parameter, i.e., fin spacing for the elliptical tubes was also carried out, which was not done in the previous study. The optimized value 
of three parameters, i.e., dimensionless tube spacing, eccentricity and dimensionless fin spacing was obtained to be $0.5,0.5$, and 0.006 respectively.

The performance of the plate-finned-tube heat exchanger in the dehumidifying condition was studied by Wang et al (1997) as we have discussed; however, it did not consider the mass transfer characteristics of the heat exchangers. Pirompugd et al (2005) investigated the performance of the plate-finned-tube heat exchanger in the dehumidifying conditions experimentally for a range of $525 \leq R e \leq 7650$. Also, they developed a tube to tube reduction method from the method of Threlkeld (1970) to analyze the performance of the heat exchanger. It was found that the heat transfer was unaffected by the varying fin pitch for 1 and 2 row coils, which is contrary to the other studies which we have discussed so far. They argued that the condensate roughens the surface and enhances mixing, which results in a negligible effect of fin pitch on the heat transfer. According to their analytical model, the mass transfer coefficient did not show any variation with the humidity; however, the Threkeld method showed some discrepancy and the mass transfer coefficient increased with an increase in the inlet humidity. The mass transfer coefficient was found to be independent of the Reynolds number for $R e<1000$, however, for $\operatorname{Re}>1000$, the mass transfer coefficient increased with an increase in the Re. As the number of rows was increased, the mass transfer coefficient decreased because of the blockage of the condensates by the tubes. The $h / j_{\mathrm{m}}$ ratio varied from 0.6 to 1.0 . However, a slight decrease was found with a decrease in the fin spacing. The correlations for the heat and mass transfer coefficients were proposed.

In the previous studies discussed, it can be noted that the heat transfer and pressure drop characteristics of the plate fin heat exchanger have been investigated with the fin spacing as the only parameter and the effect of other geometrical parameters have not been considered. Erek et al (2005) performed 3D numerical simulations to study the heat transfer and pressure drop characteristics of plate fins with varying geometrical parameters (tube center location, fin height, tube thickness, tube ellipticity and fin pitch). It was observed that, as the fin pitch was increased, the pressure drop decreased for the same tube ellipticity. The placement of the tube downstream made the horseshoe vortices more strong, which resulted in more heat transfer. The heat transfer and the pressure drop increased with an increase in the fin height. Furthermore, the heat transfer increased and the pressure drop decreased as the tube thickness was decreased and the ellipticity was increased.

Various researchers have performed studies on the performance of the air-cooled heat exchangers with respect to the parameters like, Reynolds number, fin spacing, number of tube rows, fin pitch. However to present a common essence for the variation of heat transfer with these parameters, He et al (2005) performed numerical simulations and the results were explained according the field synergy principle. The synergy is defined as the angle between the velocity and the temperature gradient. The synergy principle states that the heat transfer properties of the finned geometry depend on the intersection angle between the velocity and the temperature gradient, and by minimizing this angle we can maximize the heat transfer. 3D numerical simulations were performed with varying Reynolds number $\left(288 \leq R e_{s} \leq 5000\right)$, fin pitch $\left(0.5 \mathrm{~mm} \leq f_{p} \leq\right.$ $5 \mathrm{~mm})$, number of tube rows $\left(1 \leq N_{r} \leq 4\right)$, and dimensionless tube pitch $\left(1.2 \leq S_{t} / D \leq 3 \mathrm{~mm}\right)$. It was found that $N u$ increases with an increase in the Reynolds number, but the rate of increase became lower for $R e>1000$. The friction factor was found to decrease with an increase in the Reynolds number. The average intersection angle between the velocity and the temperature gradient (synergy) increased with an increase in Reynolds number; this indicates that the behavior of $\mathrm{Nu}$ with respect to Reynolds number was due to the change in the intersection angle. The synergy was good in the inlet region and it got worse at the back side of the tube; therefore, slits were recommended for the rear half of the fin. Both the synergy and the $N u$ were maximum for 
a fin pitch of $0.6 \mathrm{~mm}$. The synergy and $N u$ increased with an increase in the number of tube rows; however, they did not show any significant variation for $N_{r}>3$. Therefore, row number less than 3 were recommended for the practical purpose. $N u$ and synergy were found to decrease with an increase in the transverse tube pitch and as compared to the longitudinal tube pitch, the effect of the transverse tube pitch was more pronounced on the synergy and $\mathrm{Nu}$.

The enhancement of the thermal-hydraulic performance by using different types of fins and optimizing the geometry of the particular fin has been done extensively and great enhancements in the performance of the heat exchangers has been reported so far. Another way to improve the thermo-hydraulic performance is the use of electro hydrodynamic (EHD) wire electrodes. The EHD electrodes generated a high electric field in the fluid resulting in the ionization, and the ions are then driven by Coulomb force. In their path, they transfer momentum to the fluid and disturb the flow. The effect of the electro-magnetic forces on the heat transfer and friction factor has been studied for a tube, channel and tube bundles in the past. Yabe et al $(1978,1987)$, Yabe (1991), Kulacki (1983), Poulter \& Allen (1986), Nelson et al (1991), Ohadi et al (1991), Ishiguro et al (1991), Ogata et al (1992), Wangnippanto et al (2001), but Lin \& Jang (2005) were the first to apply the EHD electrodes on a finned-tube heat exchanger. They studied the effects of different arrangements of electrodes, applied voltage and tube pitch on the streamline, pressure and temperature profile for a finned tube heat exchanger $\left[D=20 \mathrm{~mm}, t_{f}=0.2 \mathrm{~mm}, f_{p}=6.2\right.$ $\mathrm{mm}, S_{l}=34.6 \mathrm{~mm}$ (for staggered arrangement), $40 \mathrm{~mm}$ (for inline arrangement), and $S_{t}=40$ $\mathrm{mm}$ ]. Four different configurations of wire electrodes were investigated. For inline arrangement, for Voltage $=0$, there was a large recirculation zone between the tubes resulting in a dead zone, and when a voltage of 4 and $16 \mathrm{kV}$ was applied, the flow became distorted resulting in more heat transfer. For the staggered arrangement, the recirculation zone was less, and the effect of the EHD was more pronounced for square formation of electrodes. For $R e=200$, the increase in the heat transfer was $10 \%$, and the increase in friction factor was $35 \%$. As the Reynolds number was increased, the effect of EHD on the heat transfer and pressure drop decreased due to the dominance of the forced convection.

Most of the studies performed on the air-cooled heat exchangers have assumed a constant temperature distribution and convective heat transfer coefficient distribution over the finned surface, but in reality the local heat transfer coefficient varies over the fin surface. Only few studies have been reported to measure the distribution of local heat transfer coefficient over a finned surface. Chen et al (2005) developed a finite difference method in conjunction with the least square method and the temperature measurements to predict the local heat transfer coefficient on the fin surface. In this method, the fin was divided into several sub-regions, and the temperature measurements were taken at particular locations on the fin. The average heat transfer was also obtained with respect to the varying air speed and tube surface to ambient temperature difference. The experiments were also performed on a finned-tube $[D=40 \mathrm{~mm}, 100 \mathrm{~mm} \times 100 \mathrm{~mm}$ (square fin), $t_{f}=2 \mathrm{~mm}$ ] and the fin was divided into six different regions with one thermocouple per region. The frontal air speed was varied from $0.3 \mathrm{~m} / \mathrm{s}$ to $6.5 \mathrm{~m} / \mathrm{s}$, and the corresponding $R e$ varied from 2500 to 13000 . It was found that the distribution of the temperature and the heat transfer coefficient was not symmetric on both sides of the tube. The upstream region had higher heat transfer coefficient than the downstream side of the fin and a wake region was found to exist at the back side of the tube. The ratio of the heat transfer coefficient at the front side to the back side was found to be 10 to 7 for different base to ambient temperature difference. Therefore, the improvements measures were recommended for the back side of the tube to enhance the heat transfer performance. The heat transfer increased and $\eta$ decreased with an increase in the base to ambient temperature difference. As the air speed was varied from 1 to $5 \mathrm{~m} / \mathrm{s}$, there was an increase in the average heat transfer coefficient and a decrease in the fin efficiency. In the similar 
manner, Chen et al (2007) applied the same method to predict the temperature distribution and average heat transfer coefficient of square fins for different air speeds and fin spacing. The heat transfer coefficient increased and the fin efficiency decreased with an increase in the fin spacing. The heat transfer coefficient and fin efficiency reached the asymptotic value as $S \rightarrow \infty$.

Pirompugd et al (2007a, b) developed a finite circular fin method (FCFM) and applied it on a plain-finned-tube heat exchanger to study the heat and mass transfer characteristics. The experiments were performed on the same setup as was used by Pirompugd et al (2005) for a range of $350 \leq R e \leq 5300$. It was observed that the fin efficiency obtained for the partial wet surface was higher as compared to the fin efficiency for the fully wet surface and was lower than the fin efficiency for the fully dry surface and the fin efficiency decreased with an increase in the relative humidity. It was also shown that the fin efficiency calculated by the enthalpy difference shows some discrepancy with the fin efficiency calculated from their presented equation, which was the combination of the fin efficiency in fully dry and fully wet conditions. Similar to their previous method (Pirompugd et al 2005), the FCFM showed some discrepancy with the method of Threlkeld (1970). The heat transfer was found to be independent of the fin pitch. The mass transfer was found to be independent of the inlet humidity at larger fin pitch, and at smaller fin pitch, it decreased with an increase in the inlet humidity. The correlations for the heat and mass transfer were also proposed. Pirompugd et al (2009) presented a review on the reduction methods for the heat and mass transfer of the finned-tube heat exchangers under dehumidifying conditions. The major comparison was presented between the models based on lumped approach e.g., Threlkeld model (1970), tube-by-tube reduction method (TTM) developed by Pirompugd et al (2005), fully wet and fully dry tiny circular fin method (WDFM) developed by Pirompugd et al (2007b), finite circular fin method (FCFM) developed by Pirompugd et al (2007a, b), and Equivalent dry bulb method (EDT) developed by Wang \& Hihara (2003). It was observed that the models based on the lumped approach (Threlkeld, EDT) were unable to predict the heat and mass transfer characteristics of the partially wet surfaces. TTM was also originated from original Threlkeld method and could not predict the heat and mass transfer characteristics of the partial wet surface. WDFM was based on the division of the finned-tube-heat exchangers into many segments and was able to predict the heat and mass transfer characteristics for surfaces with fully wet and fully dry conditions; however, the prediction for tiny surfaces with both wet and dry conditions was not possible with it. FCFM method was found to be the best among all the models, which could predict the heat and mass transfer characteristics for partial wet and dry surfaces, along with the fully dry and fully wet conditions.

Huang et al (2009) applied the SDM (steepest descent method) developed by Huang et al (2003) with commercial code CFX 4.4 to study the temperature distribution and convective heat transfer coefficient and to check the validity of the SDM. The experimental setup was the same as was used by Ay et al (2002). The results showed that, for the inline arrangement, the heat transfer coefficient was maximum only in the front of the first row tubes, and not in between the tubes as was found out by Ay et al (2002). As the air velocity was increased, the thermal boundary layer in front of the first row got thinner and the horseshoe vortices became stronger, which resulted in a higher heat transfer coefficient. As the fin pitch was increased to $15 \mathrm{~mm}$ for higher air velocity, the heat transfer coefficient increased around $2^{\text {nd }}$ and $3^{\text {rd }}$ row tubes. For the staggered arrangement, the results were almost similar to the inline arrangement, however, near the $2^{\text {nd }}$ and $3^{\text {rd }}$ row tubes the heat transfer coefficient was higher for lower fin pitch $\left(f_{\mathrm{p}}=100\right.$ $\mathrm{mm})$ as well. Overall, the heat transfer coefficient for the staggered arrangement was higher than the inline arrangement.

Xie et al (2009) argued that no study had been performed for large number of rows (more than 4) with larger diameter of the tubes (greater than $13 \mathrm{~mm}$ ). Therefore, it motivated them to 
perform numerical simulations to study the effect of number of tube rows, diameter of the tubes, tube pitch, and fin pitch on the thermo-flow characteristics of the larger diameter finned tubes for a range of $1310 \leq R e \leq 7700$. The results were validated with Kang et al (1994), and also were compared with Tang et al (2007a, b) and Gray \& Webb (1986). The heat transfer and the pressure drop were found to be independent of the number of tube rows for $N_{r}>6$. Increase in the tube diameter resulted in a decrease in the heat transfer coefficient and an increase in the pressure drop. Also, the heat transfer and the pressure drop increased with a decrease in the fin pitch. The effect of the transverse tube pitch was more pronounced as compared to the longitudinal pitch on the performance of the heat exchanger. The correlations for the friction factor and $\mathrm{Nu}$ were proposed.

Choi et al (2010) carried out experimental investigation to study the heat transfer and pressure drop characteristics of the discrete-plate-finned tubes for larger fin pitch $\left(f_{p}>8 \mathrm{~mm}\right)$. The experimental setup consisted of an open wind tunnel with an ethylene glycol-water mixture as the refrigerant on the tube side and the experiments were performed for a controlled temperature and humidity of the air. A total of 32 samples of heat exchangers were tested with inline and staggered arrangement of tubes. The Colburn factor for the discrete plate finned-tubes was found to be $6-11 \%$ higher than the continuous plate-finned-tube heat exchanger for 7.5 $\mathrm{mm} \leq f_{p} \leq 15 \mathrm{~mm}$. The effect of the number of tube rows on the Colburn factor was found to be more severe for the continuous plate finned-tube heat exchangers. The Colburn factor was higher for the staggered arrangement as compared to the inline arrangement. A correlation was proposed for the Colburn factor and it satisfied the experimental data with a deviation of about $3 \%$.

Paeng et al (2010) carried out numerical simulations and experiments to study the convective heat transfer coefficient for the plate fins for $1082 \leq R e \leq 1649$. The experimental setup consisted of an evaporator, a compressor, a condenser and an expansion valve. The refrigerant R-22 was used as the working fluid on the tube side. A correlation for $N u$ was derived. The results were compared with the results of the Kays \& London (1955), Wang et al (2000), Gray \& Webb (1986), and Kim et al (1999). The relative error between the results of Paeng et al (2010) and Kim et al (1999) was found to be in $6 \%$ range, whereas the relative error with the other three published data ranged from 10 to $32 \%$.

Chen \& Lai (2012) applied the inverse scheme of the finite difference method in conjunction with the least square method to predict the temperature distribution and average heat transfer coefficient of two row plate finned-tube heat exchanger for different air speed and fin spacing. The experiments were performed in the similar manner as were performed in their previous study (Chen et al 2005). The fin was divided into 12 regions, and one thermocouple was placed in each region to measure the surface temperature. It was observed that the heat transfer coefficient increases with an increase in the air velocity and fin spacing; however, it reaches its asymptotic value at as $S \rightarrow \infty$. The results were in good agreement with Rosman et al (1984), and Saboya \& Saboya (2001) for $0.5 \leq U_{f r} \leq 1.5$.

3.2b Wavy fins: Wavyfin pattern (figure 3B) provides a longer airflow path with boundary layer breakage at the crest of the fin, and therefore, increases the heat transfer rate. Beecher \& Fagan (1987) tested 27 fin-and-tube heat exchangers, 21 of them having wavy fin geometry with three rows in staggered arrangement. Webb (1990) used a multiple regression technique to correlate Beecher and Fagan's data. Webb's correlation was able to predict $88 \%$ of the wavy fin data within $\pm 5 \%$, and $96 \%$ of the data was correlated within $\pm 10 \%$. However, the wavy fin geometry tested by Beecher and Fagan was a little different from the commercially used wavy fins. These 
previous studies were performed with a consideration of constant temperature on the fin surface and neglecting the contact resistance between fins and tubes. The effect of dehumidifying conditions on the heat transfer and pressure drop characteristics of air-cooled heat exchangers has been studied by many researchers in the past. For some of the researchers like Bettanini (1970), Guillory \& McQuiston (1973), Myers (1967), Elmahdy (1975), Eckels \& Rabas (1987), Yoshii et al (1973) and McQuiston (1978a, b), the heat transfer coefficient was higher in the wet conditions as compared to the dry conditions. On the other hand for Tree \& Helmer (1976), the heat transfer characteristics obtained under dry and wet conditions were the same, and for Jacobi \& Goldschmidt (1990), the heat transfer coefficient was lower for the wet surface as compared to the dry surface. Mirth \& Ramadhyani (1993) tried to clarify this discrepancy by performing experiments on different samples of the wavy-finned-tube heat exchangers under wet conditions for a range of $300 \leq R e \leq 1700$. A model was developed in order to evaluate the heat transfer performance by discretizing the heat exchanger coil into many segments. The results for the wet coil were compared with the correlations for the dry coils, and the results indicated that the wet surface $\mathrm{Nu}$ showed some deviation with the correlation for dry coils. The extent of discrepancy was different for different coils and no conclusion could be drawn from that. To resolve the issue, the sensitivity analysis was carried out, and it was found that small changes in the heat transfer (air side) and dew point temperature affected the results for $\mathrm{Nu}$ by a large amount, which resulted in a randomness in the results. The effect of the change in the temperature of inlet air and inlet water was negligible. The use of the dry surface correlation for the wet surface $N u$ predicted the heat transfer within $\pm 5 \%$. In the similar study, Mirth \& Ramadhyani (1994) presented the Nusselt number data for the dry surface and wet surface and presented the correlation for $\mathrm{Nu}$ in the dry conditions. The effect of the fin pitch, coil length, and tube rows was explained in a greater detail and two correlations for the friction factor were presented for two different sets of coils. The friction factor correlations for the first set of coil included the effects of fin spacing, length of the coil, whereas the correlation for the other set was independent of the coil length. $\mathrm{Nu}$ was found to be higher for short coils and this was attributed to the developing flow region length in the shorter coils. $\mathrm{Nu}$ increased with an increase in the fin spacing.

Wang et al (1997) performed experiments to study the heat transfer and pressure drop characteristics for 18 samples of the wavy-finned-tube heat exchangers. The experimental setup was set according to the ASHRAE 41.1 guidelines. For the staggered arrangement, the heat transfer decreased with an increase in the number of rows for $R e<900$, and for $R e>900$, and the heat transfer increased with an increase in the number of tube rows. However, the friction factor did not show much variation with the number of tube rows. For the inline arrangement, the heat transfer first increased with the number of rows for $R e<900$, but for $R e>900$, the effect of number of rows became insignificant.

Madi et al (1998) argued that some parameters have not been investigated in the past for the plate fin and the wavy fin. Therefore, they considered 28 samples of heat exchangers consisting of the plain and the wavy fin and studied the effect of fin spacing, number of tube rows, fin thickness and tube pitch on the thermal-hydraulic performance of the heat exchangers. They proposed the correlations for the Colburn factor and the friction factor. The experiments were performed in an open wind tunnel with a pitot tube for the measurement of the velocity and a mesh of thermocouples for the temperature measurement. The wavy fin was found to have a larger the Colburn factor and the friction factor as compared to the plain fins. The fin thickness of $0.12 \mathrm{~mm}$ showed a better heat transfer performance over a fin thickness of $0.13 \mathrm{~mm}$; however, the effect of the fin thickness on the friction factor was negligible. The effect of the number of tube rows on the Colburn factor was found to be influenced by the heat exchanger geometry 
and the Reynolds number. In this study, no physical explanation was given for the effect of the different parameters.

The effect of the waffle height $\left(h_{\mathrm{w}}=1.18 \mathrm{~mm}\right.$ and $\left.1.58 \mathrm{~mm}\right)$ on the thermal-hydraulic performance of herringbone wavy fins under dehumidifying condition was first investigated by Wang et al (2000b). For 2-row coil, the increase in the pressure drop (with respect to the plain fins) was $150 \%$ higher for a larger waffle height in the dehumidifying conditions and about $75 \%$ higher in the dry conditions. The effect of the waffle height on the heat transfer was found to be negligible. The reason for the increase in the pressure drop was found to be the blockage of the condensate and formation of a larger vortex for the larger waffle height, and it was also observed that the pressure drop decreases with an increase in the fin pitch. For 4-row coil, the effects were similar but were less pronounced for the pressure drop, because the flow became fully developed as the number of rows was increased. The heat transfer was found to be a strong function of the fin pitch for larger waffle height.

Wongwises \& Chokeman (2004) were the first one to study the effect of fin thickness $(0.115$ $\leq t_{f} \leq 0.250 \mathrm{~mm}$ ) for the wavy fins for a range of $900 \leq R e \leq 5400$. They performed experiments in a wind tunnel and water was used on the tube side with a fixed inlet temperature ranging from 55 to $65^{\circ} \mathrm{C}$. It was found that for $N_{r}=2$, the Colburn factor increases with an increase in the fin thickness due to the occurrence of the horseshoe vortices at the leading edge, and the friction factor also increased with the fin thickness for $f_{p}=1.41 \mathrm{~mm}$ and $1.81 \mathrm{~mm}$; however, for $f_{p}=2.54 \mathrm{~mm}$, the effect of the fin thickness on the friction factor was negligible. For $N_{r}=4$, for $R e<1800$, the Colburn factor decreased with an increase in the fin thickness due to the dominance of the wake region over the formation of horseshoe vortices; however, for $R e>1800$, the formation of the horseshoe vortices dominated over the formation of the wake region and it resulted in an increase in the Colburn factor with an increase in the fin thickness. For $N_{r}=4$, the friction factor was independent of the fin thickness for $f_{p}=2.54 \mathrm{~mm}$; however, for $N_{r}=6$, the friction factor decreased with an increase in the fin thickness. Wongwises \& Chokeman (2005) continued their research on the wavy fin and studied the effect of fin pitch and number of tube rows on the thermal-hydraulic characteristics of the wavy-finned-tube heat exchanger. The experimental setup was the same as was used in their previous study (Wongwises $\&$ Chokeman 2004). The results showed that, the Colburn factor decreases with an increase in $R e$ and the effect of the fin pitch on the Colburn factor was negligible for all the values of $R e$. As the fin pitch was increased, the mixing in the flow enhanced, which resulted in an increase in the Colburn factor, however, the recirculation zone downstream of the wavy apex also extended leading to a decrease in the Colburn factor. These both effects cancelled each other, and as a result, the Colburn factor remained constant. The friction factor was higher for the larger fin pitch at low Reynolds number $(R e<2000)$, and as $R e$ was increased, the friction factor for the lowest fin pitch $(1.41 \mathrm{~mm})$ became maximum. The Colburn factor decreased with an increase in the number of tube rows for $R e<4000$, and as the Reynolds number was increased further, the effect of number of tube rows diminished. The friction factor was highest for the lowest number of rows. Chokeman \& Wongwises (2005) performed experiments to study the effects of fin pattern and edge corrugation on the thermal-hydraulic characteristics of the wavy-finned-tube heat exchanger and performed experiments for a range of $1400 \leq R e \leq 5600$. The experiments were performed on the similar setup as was used by Wongwises \& Chokeman (2005). Two different fin configurations and edge corrugation were considered in the study and it was found that fin 2 provides a better heat transfer coefficient and larger pressure drop as compared to the fin 1 . The enhancement in the heat transfer coefficient was believed to be caused by the reduction in the wake region behind the tubes for fin 2 . The enhancement in the heat transfer coefficient and pressure drop was 5.25-15.39\% and 0-6.61\%, respectively, and fin 2 was recommended for 
use in the commercial heat exchangers. The heat transfer coefficient decreased by $8.93 \%$ when edge corrugation was present; however, no effect on the pressure drop was observed. Therefore, it was recommended not to use the edge corrugation in the cases where durability of the heat exchanger does not matter much.

As we have discussed in Section 3.2a, Pirompugd et al (2005) performed experiments on the plain-finned-tube heat exchangers in the dehumidifying conditions. In the similar manner, Pirompugd et al (2006) carried out experiments on the wavy-finned-tube heat exchangers in the dehumidifying conditions. The experimental setup and geometrical parameters were the same as in their previous study. It was observed that the fin spacing affects the heat transfer and mass transfer for $N_{r}=1$, and $R e<3000$, whereas for $R e>3000$, the effect of fin spacing reduces, and the flow becomes vortex dominated. The wave height also affected the heat transfer coefficient for smaller fin pitch. The mass transfer coefficient showed some variation with the inlet humidity at lower fin pitch, and the reason was believed to be the blockage of the flow path by the condensate. For $N_{r}=2$, both the mass transfer coefficient and the heat transfer coefficient decreased, and the effect of the inlet humidity and fin spacing reduced. For $N_{r}=4$ and 6, the effect of the geometrical parameters and the inlet conditions became less pronounced. The ratio of the heat transfer coefficient to the mass transfer coefficient varied from 0.6 to 1.1 , and was insensitive to the fin pitch. The correlations for the heat and mass transfer coefficients were given.

Earlier in this section, we discussed that Wongwises \& Chokeman (2004) studied the effect of the fin thickness on the performance of the wavy-finned-tube heat exchangers in the dry conditions. Kuvannarat et al (2006) carried forward their research in the dehumidifying conditions. The experimental setup was same and air was kept at a constant temperature of $28^{\circ} \mathrm{C}$ with a $60 \%$ relative humidity. It was observed that for small fin pitch $\left(f_{\mathrm{p}}=1.41 \mathrm{~mm}\right)$, the heat transfer coefficient for $t_{f}=0.25 \mathrm{~mm}$ was $5-50 \%$ higher than for $t_{f}=0.115 \mathrm{~mm}$, and the corresponding pressure drop was $5-20 \%$ higher for $t_{f}=0.25 \mathrm{~mm}$. However, at higher fin pitch, the effect of the fin thickness was not pronounced. This was due to the condensate present in the heat exchanger. At low fin spacing, the size of the condensate droplets was comparable to the fin spacing; therefore, the swirling motion produced by the droplet mixed well with the main flow leading to a higher heat transfer rate. However, at higher fin spacing, the swirling motion produced by the droplets was not able to mix well with the main flow, and as a result, the effect of the fin thickness became negligible. Also, the effect of the fin thickness became negligible with an increase in the number of tube rows to 6 . The correlations for the Colburn factor and friction factor were given.

Tao et al (2007a) carried forward the study of He et al (2005) (discussed in Section 3.2a) and performed the $3 \mathrm{D}$ numerical simulation to study the thermal-hydraulic characteristics of the wavy fin for varying wavy angle $\left(0^{\circ} \leq \theta \leq 20^{\circ}\right)$ (figure 3B). It was observed that, $N u$ increases and the friction factor decreases with an increase in $R e$. Nu was found to be higher on the front side and lower on the back side of the tube and it decreased along the flow direction. An increase in the fin area on the front side and decrease in area on the back side of the tube was recommended for better performance of the heat exchanger.

Cheng et al (2007) investigated the wavy fin and tube heat exchanger numerically. The main aim of the study was to simulate the heat exchanger with larger number of tube rows and to use the synergy principle to explain the results. Periodic boundary condition was applied at the inlet and outlet, so that a larger number of tube rows could be included. The wavy angle was varied in the range of $0^{\circ} \leq \theta \leq 35^{\circ}$. All the results were explained by the synergy principle. The effect of the wavy angle was also explained and the synergy angle was found to be minimum for the largest wavy angle, and hence the heat transfer rate was maximum for the largest wav angle.

In section 3.2a, we discussed the finite circular fin method developed by Pirompugd et al (2007a, b) for a plain fin. Pirompugd et al (2008) applied the same method on the wavy-finned- 
tube heat exchanger for a range of $500 \leq R e \leq 5000$. The results were almost similar to the results obtained for the plain fin by Pirompugd et al (2007a, b). For $2 \leq N_{r} \leq 4$, they observed that the inlet relative humidity does not affect the heat transfer characteristics. It was found that the mass transfer coefficient decreases with an increase in the fin spacing for 1-row coil, and as the number of rows was increased, the effect of the fin spacing disappeared. The reason was the same as was explained for the plain fin in the previous study. It was also observed that the mass transfer decreases with an increase in the inlet relative humidity at low fin spacing. The correlations were proposed for the heat and mass transfer characteristics.

Cheng et al (2009) carried forward their previous study (Cheng et al 2007) on the wavy fin and tube heat exchanger. The numerical model and the boundary conditions were the same as used in Cheng et al (2007). The friction factor was found to be larger for the larger wave amplitude, and it decreased with an increase in the Reynolds number. For a larger fin pitch, they obtained a lower friction factor and a higher $\mathrm{Nu}$; however, the effect was reversed at low Reynolds number for $N u$. Both $N u$ and the friction factor were higher for a larger diameter of the tube $(D=13.36$ $\mathrm{mm}$ ); however, the increase in the friction factor was higher than the increase in $N u$. The friction factor for four waves was very large as compared to the friction factor for one and two waves, similar effect was observed for $\mathrm{Nu}$.

Tao et al (2011) performed numerical simulations to study the effect of various parameters (Reynolds number, fin pitch, wavy angle, fin thickness, transverse tube pitch) on the heat transfer and pressure drop characteristics of the wavy-finned-tube heat exchanger. To validate the selfdeveloped code, the results were compared with Wang et al (2002) and Xin et al (1994). It was observed that, $\mathrm{Nu}$ increases and the friction factor and fin efficiency decrease with an increase in the Reynolds number. Increase in the wavy angle resulted in an increase in $\mathrm{Nu}$ and the friction factor, and an optimized wavy angle $10^{\circ} \leq \theta \leq 20^{\circ}$ was recommended. $N u$ increased with an increase in the fin pitch up to a certain fin pitch $\left(f_{\mathrm{p}}=1.6 \mathrm{~mm}\right)$ and then started decreasing, and the friction factor decreased with an increase in the fin pitch. An optimum value of fin pitch between $1.2 \mathrm{~mm}$ and $2 \mathrm{~mm}$ was recommended for the practical use. As the fin thickness was increased, $\mathrm{Nu}$, the pressure drop, and the fin efficiency increased. Increase in the transverse tube pitch decreased the fin efficiency, the heat transfer and the pressure drop.

3.2c Fins with vortex generators: In this section, we are going to discuss about the thermalhydraulic properties of the fins with vortex generators (figure $3 \mathrm{C}$ ). Four types of vortex generators $(\mathrm{VGs})(\mathrm{DW}=$ delta wing, $\mathrm{RW}=$ rectangular wing, $\mathrm{RWP}=$ rectangular winglet pair, $\mathrm{DWP}=$ delta winglet pair) are shown in figure $5 \mathrm{~A}$ and in figure $5 \mathrm{~B}$, we have shown the dimensions of the delta winglet ( $c=$ length, $b=$ span of the delta winglet, $\alpha=$ attack angle). The vortex generators produce longitudinal and transverse vortices which enhances the heat transfer on the air side. Brockmeier et al (1993) performed numerical simulations to compare the performance of the fin with delta wings with the plain fin, off-strip fin, and louvered fins for a range of $500 \leq R e \leq 3000$. The experimental data for the plain fins, off-strip fins and louvered fins was obtained from the literature and was compared with the numerical results obtained for the fin with delta wings. It was found that the fin with delta wing performs best out of all fin configurations. The flow became periodically fully developed after a certain downstream distance, and the horseshoe vortices were observed at the leading edge of the winglet. The fin with delta winglet allowed a $76 \%$ decrease in the heat transfer area as compared to the plain fins.

Fiebig et al (1993) performed experiments on the inline and staggered bank of finned tubes with delta winglets to study the heat transfer and pressure drop characteristics. The experiments were performed in a wind tunnel with a three row heat exchanger. The temperature measurements were performed with the help of the liquid crystal thermography. Without the VGs, the average 


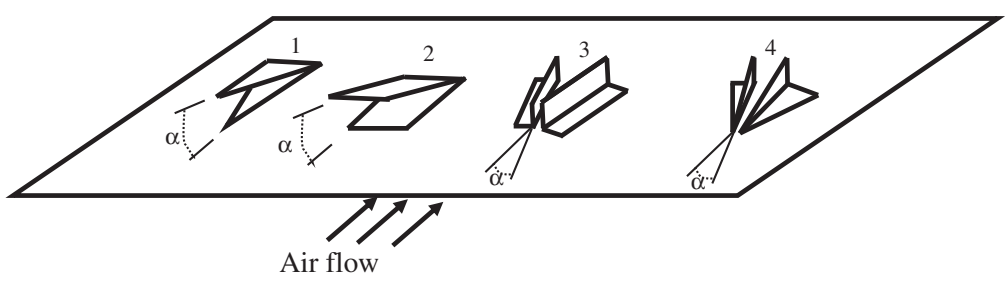

(A)

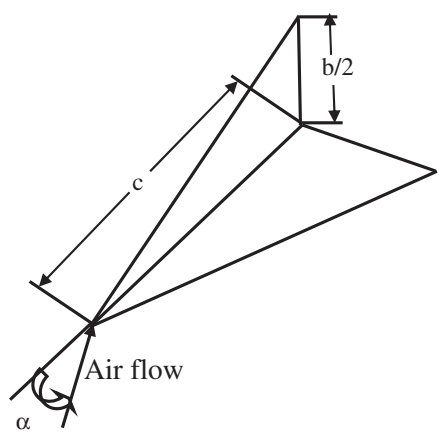

(B)

Figure 5. Schematic of (A) Vortex generators, (1) delta wing, (2) rectangular wing, (3) rectangular winglet pair (4) delta winglet pair, (B) Delta winglet, $\mathrm{c}=$ length, $\mathrm{b}=$ span, $\alpha=$ attack angle.

$\mathrm{Nu}$ was higher for the staggered arrangement as compared to the inline arrangement, but with the VGs, $N u$ was higher for the inline arrangement as compared to the staggered arrangement. With the VG, for the inline arrangement, the increment in $\mathrm{Nu}$ and friction factor was $55-60 \%$ and $20-45 \%$, respectively, while for the staggered arrangement, the increment in the $\mathrm{Nu}$ and friction factor was $9 \%$ and $3 \%$, respectively.

Previous two studies focused on the heat transfer enhancement by the longitudinal vortices; however, the effect of the transverse vortices produced by the VGs was not considered. To study the interaction between the longitudinal and the transverse vortices, Biswas et al (1994) performed numerical simulations in a rectangular channel with tube and winglet type vortex generator (DWP) for a range of $500 \leq R e \leq 1000$. The Marker and cell method was used for the solution of the equations, and hybrid scheme was employed for the discretization. The longitudinal vortices were observed near the tube but the transverse vortices could not be observed. The flow became periodic for $R e=1000$, and the periodicity was attributed to the interaction of the transverse and the longitudinal vortices. $\mathrm{Nu}$ was higher for the fin with DWP as compared to the plain fin, and the maximum increment was $240 \%$ at any axial location.

Fiebig et al (1994) performed the experimental investigation to study the heat transfer and pressure drop characteristics of the plate-finned-flat-tubes with DWP, and the results were compared with the results of plate-finned-round-tubes with DWP. The experimental setup was the same as was used by Fiebig et al (1993). Without the DWP, $N u$ was higher for the round tubes as compared to the flat tubes. With the DWP, $N u$ increased by $80-120 \%$ for the flat tubes; however, it increased by only $10 \%$ for the round tubes. With the DWP, the friction factor increased by two times for the flat tubes, but it was still lower than the friction factor offered by the round tubes with the DWP. Overall, the flat tubes with the DWP provided two times the heat transfer and half the friction factor as compared to the round tubes with the DWP. The longitudinal vortices 
produced by the vortex generators are helpful in enhancing the heat transfer from any heat transfer surface and in the previous studies of Fiebig and coworkers, the longitudinal vortices generated by a delta winglet have been studied. Tiggelbeck et al (1994) continued their work and compared the thermal hydraulic performance of a plate fin with four types of vortex generators e.g., DW, RW, DWP and RWP. The range of $R e$ was from 2000 to 9000 and the angle of attack was varied from 30 to $90^{\circ}$. It was observed that, the DWP performs best in terms of averaged $N u$ followed by the RWP; however, the drag coefficient was also highest for the DWP. The performance of the DWP and the RWP was studied at various attack angles, and it was found that the maxima in the $N u$ occurs between $50^{\circ}$ and $70^{\circ}$ for the DWP and between $45^{\circ}$ and $65^{\circ}$ for the RWP. However, the maxima for the DWP was much larger. The drag coefficient kept on increasing as the attack angle was increased.

Fiebig et al (1995) performed numerical investigation to study the effect of the DWP on the heat transfer and the heat transfer reversal for a plate-fin-tube heat exchanger for a range of 250 $\leq R e \leq 500$. It was shown that the DWP produces longitudinal vortices due to the pressure difference on the two sides of the winglets. The longitudinal vortices helped the air flow to go into the wake region of the tube, as a result, the heat transfer reversal was eliminated and the overall heat transfer increased by $31 \%$.

Jacobi \& Shah (1995) presented a review on the use of the longitudinal vortices as a method of the heat transfer enhancements. They reviewed the active and passive vortex methods for the enhancement of the heat transfer. In the Passive vortex method, studies on the mixed and forced convection in rectangular channels, channels with single tube in cross flow, and channels with multiple tubes were presented. In the active vortex methods, the use of skewed and pitched wall jets, electrohydrodynamics (EHD) and acoustic streaming was suggested. Based on the reviews of recent literature, a lack in the fundamental research on the vortex-induced air-side heat transfer enhancement was found. The understanding of the flow pattern in the practical heat exchanger designs, which operated in the laminar region at that time was found to be missing. The experimental studies on the heat exchanger at low $R e$ were suggested for the future work, and it was also suggested that further studies should be carried out with three DWPs in the flow direction with more than three passages in the transverse direction. Further studies were suggested to compare the performance of the vortex generators with the performance of the strip and the louver fins. Chen et al (1998a) performed numerical investigation to study the effect of the DWP on the thermal-hydraulic characteristics of the finned oval tubes for different attack angles $(\alpha$ in figure 5B) and aspect ratio (AR) of the DWP. The number of the rows of delta winglets was varied from 1 to 3 and the value of $R e$ was 300. The longitudinal and corner vortices were observed, which were caused by the leading edge and the trailing edge of the winglets, respectively. The best performance of the DWP was observed for an $A R$ of 2 and an attack angle of $20^{\circ}$.

Fiebig (1998) presented a survey on the delta and the rectangular vortex generators. The formation of the longitudinal and transverse vortices and their effect on the heat transfer enhancement was given in detail. The comparison of the rectangular and triangular vortex generators was also provided. The main conclusions of the survey were:

(i) The longitudinal vortices are more significant in enhancing the heat transfer as compared to the transverse vortices.

(ii) The triangular and rectangular winglets give similar performance if all other dimensionless parameters are kept same.

(iii) Heat transfer enhancement is higher in the laminar flow as compared to the turbulent flow.

(iv) The maximum heat transfer occurs at an attack angle of $20^{\circ}$. 
Global heat transfer enhancement of more than $100 \%$ over 40 times the vortex generator area was achieved.

From all the previous studies discussed, it can be observed that the heat transfer increases as the number of rows of the DWP increases. To find out the reason behind this, Chen et al (1998b) carried out numerical investigation to study the heat transfer enhancement due to the number of rows (3 rows in inline arrangement) of the DWP for a $R e$ of 300 . The interaction of the longitudinal vortices from the DWP of the first row with the longitudinal vortices caused by the DWP of the downstream rows was also explained. It was found that the longitudinal vortices produced by the DWP of the first row went through the winglets of the second row, and the longitudinal vortices of both the rows merged together and then proceeded to the third row. After third row, the longitudinal vortices from all the rows merged together. The heat transfer enhancement was maximum by the second row DWP; however, the corresponding pressure drop was also maximum. Overall, the heat transfer increased with an increase in the number of rows of delta winglets. After studying the inline arrangement of the DWP, Chen et al (2000) studied the staggered arrangement of the DWP. The numerical procedure and assumptions were the same as of their last study (Chen et al 1998a). They studied five different configurations with four in the staggered arrangement and one in the inline arrangement. The secondary flow was observed from the two DWPs in the staggered arrangement, and the secondary flow was weaker for the DWP close to the tube wall, as compared to the DWP far from the tube surface. The reason was found to be the interaction of the flow generated by the tube with the flow generated by the DWP closer to the tube wall. The pressure gradient was higher for the DWP far from the tube, and it increased for the downstream DWP. The staggered arrangement of the DWPs was more effective as compared to the inline arrangement, and the increase in the heat transfer by the staggered arrangement was $20 \%$ more with $14.6 \%$ lower pressure drop penalty as compared to the inline arrangement. Overall, the DWP far away from the wall was more effective as compared to the DWP closer to the tube wall.

Earlier in this section we have discussed the studies performed by Fiebig and group to investigate the effect of vortex generators on the thermal-hydraulic performance of the air-cooled heat exchanger. Fiebig and Group used the common flow down configuration of the DWPs, in which the leading edges of the DWP was closer than the trailing edges. The enhancement in the heat transfer has been found in the range of $10-25 \%$, and the enhancement in the pressure loss has been found in the range of $20-35 \%$ which was not so effective. Further, at low Reynolds number, the common flow down configuration decreases the heat transfer in the wake region. Therefore, to make the vortex generator more effective, Torii et al (2002) changed the common flow down configuration to common flow up configuration (figure 6) and studied the heat transfer and pressure drop characteristics of the new configuration for a range of $350 \leq R e_{l w} \leq 2100$. In the common flow up configuration, the trailing edges of the winglet pair are closer than the leading edges. The experiments were performed in a wind tunnel with a test section of dimensions $150 \mathrm{~mm} \times 100 \mathrm{~mm} \times 300 \mathrm{~mm}$. It was found that the presented common flow up configuration produces vortices, turbulence and flow acceleration between the tube and the winglet, and the flow acceleration causes the separation delay and reduces the form drag. This resulted in an enhancement in the heat transfer by $30-10 \%$ for the staggered arrangement, and $20-10 \%$ for the inline arrangement, and the corresponding decrement in the pressure drop was $55-34 \%$ for the staggered arrangement and 15-8\% for the inline arrangement.

Kwak et al (2002) carried out experimental investigation to study the effect of the DWP on the heat transfer enhancement and flow characteristics of a plate-finned-tube heat exchanger. The experiments were performed on the same setup as was used in the study of Torii et al (2002) for a range of $300 \leq R e_{h} \leq 2700$. The study was carried out in two parts, in the first part, the 
plate fins were used without DWP, and in the second part, plate fins with DWP were used in the inline and staggered arrangement. For plate fins without the DWP in the inline arrangement, the heat transfer increased very rapidly as Reynolds number was increased for $R e_{h}>1000$, and the effect of the number of rows of tubes was not so prominent. Similar effect was observed on the pressure drop, but the increase in the pressure drop was more rapid as compared to the heat transfer. For the staggered arrangement, the increase in the heat transfer and pressure drop was larger as compared to the inline arrangement with an increase in the Reynolds number. Overall, it was found that the inline arrangement performed better than the staggered arrangement and due to this reason, the DWPs were included in the inline arrangement. An increment of 10-25\% in the heat transfer and 20-30\% in the pressure drop was found with DWP included.

ElSherbini \& Jacobi (2002) argued that, in all the previous studies prior to 2002, real size heat exchangers were not used because of the experimental limitations, geometrical considerations and other restrictions. Therefore, ElSherbini \& Jacobi (2002) performed experiments to determine the effects of two sizes of delta wingson the thermal-hydraulic performance of the plate-finned-tube heat exchangers for a range of $700<R e_{\mathrm{h}}<2300$. The tests were conducted in a closed circuit wind tunnel with ASME standard orifice plate to measure the air flow rate. The recommended $A R$ of 1 and an attack angle of $55^{\circ}$ were used for the wings. Both the configurations of wings enhanced the heat transfer but no enhancement in the pressure drop was observed which was very strange. It was believed that, the wings enhance the drag but reduce the pressure drop in the wake region of the tube by delaying the separation of the flow on the tubes, and due to this, the two effects cancel each other. An overall enhancement of $29-33 \%$ in the heat transfer was observed for one type of configuration of wings and by 17-20\% for the second configuration. We have discussed that Torii et al (2002) studied the common flow up configuration of the DWP in one row with three rows of plate-finned tube heat exchanger. They obtained excellent results for the common flow up configuration as compared to the common flow down configuration of the DWP.

Kwak et al (2003) performed another experimental investigation to study the effect of number of tube rows $\left(2 \leq N_{r} \leq 5\right)$ on the heat transfer and pressure drop of plain-finned-tube heat exchanger with one row of DWP in common flow up configuration. The experimental setup and Reynolds number range were the same as in the previous study. The heat transfer for the plain-finned-tube was found to be independent of the number of tube rows, whereas the pressure drop varied slightly, and the pressure drop was maximum for the five-row coil and minimum for

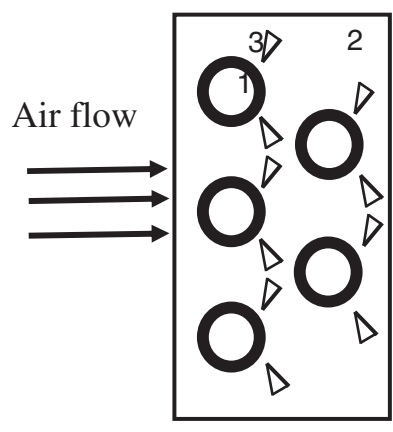

(A)

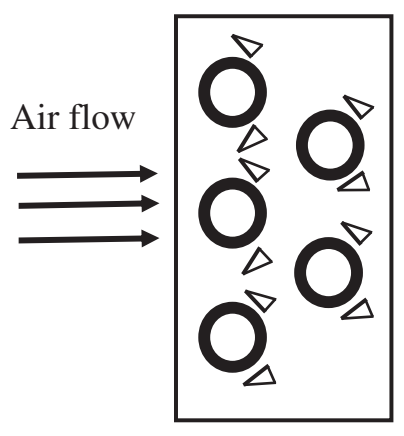

(B)

Figure 6. Delta winglet pair configurations, (A) common flow down, (1) tube surface, (2) plate fin surface, (3) delta winglet, (B) common flow up. 
the three-row coil. The heat transfer and pressure drop results of the finned tubes with DWPs were compared with the plain-finned-tube, and it was found that the ratio of the heat transfer enhancement was almost independent of the number of tube rows, except 2-row coil, which showed a maximum increment in heat transfer of 20-35\%. The increment in the pressure loss was minimum for the three row heat exchanger. Overall, the three-row coil performed best with a $30-10 \%$ augmentation in heat transfer and a reduction of $55-34 \%$ in the pressure drop for Reynolds number ranging from 350 to 2100.

Kwak et al (2005) carried forward their study and performed experiments to study the common flow up configuration of the DWP with two rows of DWP placed in a three row platefinned-tube heat exchanger. The geometric parameters were the same as were in the previous study (Torii et al 2002). It was observed that, for one row of the DWP, the results were similar to the results of Torii et al (2002), and inline arrangement performed better than the staggered arrangement. For two rows of the DWP placed in common flow up configuration, the enhancement in the heat transfer was found to be 6-15\% with a $61-117 \%$ increase in pressure loss penalty for the staggered arrangement of the tubes as compared to the one row of the DWP. For the inline arrangement of the tubes, increase in the heat transfer was 7-9\% with a 3-9\% increase in the pressure loss. Therefore, it was concluded that the common flow up configuration performed better for the inline arrangement.

Pesteei et al (2005) performed experiments to study the best location of the DWP for the plainfinned-tube heat exchanger with only one tube included in the experiments with the plain fins for a $R e$ of 2250. It was found that the DWP does not affect the upstream heat transfer coefficient, and only small change was observed in the heat transfer performance upstream; however, the downstream heat transfer coefficient was greatly enhanced. The heat transfer coefficient and pressure drop varied depending on the location of the winglets around the tube, and the best location for the winglet was at $\Delta x=0.5 D$ and $\Delta y=0.5 D$, where $\Delta x$ and $\Delta y$ were the streamwise and cross-stream distances.

Joardar \& Jacobi (2008) performed experimental investigation to study the effects of one row and multi-rows of DWP on the thermal-hydraulic performance of the plain-fin-tube heat exchanger. The experiments were performed in a closed loop wind tunnel with a cross-section of $101.6 \mathrm{~mm} \times 610 \mathrm{~mm}$, and the data reduction and interpretation was performed using ANSI/ASHRAE standards (33-2000). The DWP was put in the common flow up $\left(\alpha=15^{\circ}\right.$, $A R=1.35)$ configuration with the inline arrangement of the tubes. The results showed that with one row of the DWP, the enhancement in the heat transfer was 11.7-32.7\%, whereas with three rows of the DWP, the enhancement was 38\%. The enhancement in the pressure drop was $12 \%$ with one row of the DWP, and $87.5 \%$ at $R e=220$, and $26 \%$ at $R e=960$ for the three rows of DWP. The $j / f$ factor was also higher (35.7\% to 50.8\%) for one row of the DWP as compared to the three rows of the DWP; however, the volume goodness factor was better for the three rows of the DWP. The variation in the attack angle was recommended for better performance of the heat exchanger.

The use of the DWP was restricted to the plain fins only, until Tian et al (2009) studied the heat transfer and pressure drop characteristics of the wavy fin with the DWP in the inline and the staggered arrangements. The wavy angle was $15^{\circ}$ and the delta winglet had a length and height of $5 \mathrm{~mm}$ and $2.5 \mathrm{~mm}$, respectively. It was observed that the DWP generates a main vortex at its leading edge and a corner vortex at the junction of its face and the surface of the fin. For the inline arrangement, the longitudinal vortices went a long way downstream, because no disturbance was present, and it increased the heat transfer coefficient of the fin surface as well as of the tube surface. For the staggered arrangement, the longitudinal vortices were disrupted by the tubes, and the enhancement in the heat transfer was mainly in the wake region, and found 
to be significant only for the third row. The maximum heat transfer was observed to be $80 \%$ and $95 \%$ for the inline and staggered arrangements, respectively, as compared to the case where no DWP was present. However, the DWP increased the pressure drop as well, and the $j / f$ ratio varied from 1.46 to 1.87 for the inline and staggered arrangements respectively.

The optimization of the parameters of finned-tube heat exchangers with DWP had been performed; however, the number of parameters has always been limited. A study which includes all the geometrical parameters for a complete optimization with small number of tubes and small diameter tubes was first performed by Zeng et al (2010). All the parameters, fin pitch $(2.5 \mathrm{~mm}$ $\left.\leq f_{p} \leq 4.5 \mathrm{~mm}\right)$, fin thickness $\left(0.2 \mathrm{~mm} \leq t_{f} \leq 0.4 \mathrm{~mm}\right)$, transverse tube pitch $\left(38 \mathrm{~mm} \leq S_{t} \leq\right.$ $54 \mathrm{~mm})$, longitudinal tube pitch $\left(32 \mathrm{~mm} \leq S_{l} \leq 40 \mathrm{~mm}\right)$, vortex generator height $(1.7 \leq \mathrm{b} / 2$ $\leq 2.5 \mathrm{~mm})$, length $(4 \mathrm{~mm} \leq c \leq 6 \mathrm{~mm})$ and attack angle $\left(30^{\circ} \leq \alpha \leq 60^{\circ}\right)$ were optimized in the study. Taguchi method (Taguchi et al 1989; Taguchi 1991) was applied in the study to optimize the parameters and the numerical simulations were carried out using FLUENT software. It was observed that the copper fins show a higher heat transfer and fin efficiency as compared to the aluminum fins. As the attack angle was increased, the heat transfer and pressure drop both increased, and an intermediate attack angle $\left(\alpha=45^{\circ}\right)$ was suggested. The same effect was seen for the winglet length and height, and an intermediate value of both the parameters was suggested. The fin thickness effects were negligible and were neglected and the heat transfer decreased with an increase in the fin pitch. For the same $R e$, the heat transfer increased with an increase in the transverse tube pitch and the heat transfer and pressure drop both increased with an increase in the longitudinal tube pitch. The six factors, fin pitch, longitudinal tube pitch, transverse tube pitch, DWP length, DWP height, and attack angle were found to be the important factors affecting the performance of the heat exchanger. Based on the signal to noise ratio ( $\mathrm{SN}$ ratio), an optimized configuration was suggested.

3.2d Slit fins: The slit fins are shown in figure 3D. The basic understanding of the slit fins was presented by Mullisen \& Loehrke (1986), Mochizuki et al (1987), Dejong \& Jacobi (1997), and Zhang et al (1997). Some of the studies on the slit fins were performed by comparing the performance of slit fins with the other fins; therefore, we have presented those studies in Section 3.3. The lack of the experiment methodology in determining the local conjugate heat transfer coefficient between the fin and tube motivated Tsai \& Sheu (1998) to perform the numerical simulations to determine the local conjugate heat transfer and pressure drop for the plain and slit fins for a range of $367 \leq R e_{\mathrm{h}} \leq 1133$. The flow structure on the plain-finned-tubes was explained in detail. The spanwise average pressure decreased slowly as the tube surfaces was approached and the pressure drop was nearly independent of the $R e_{\mathrm{h}}$ in the region where tubes were present. The heat transfer increased as the velocity was increased and the flow separation could be observed behind the tubes resulting in a wake region and a jet-like accelerating flow. The heat transfer was very poor in the wake region but in the upstream region of the tube, the heat transfer was very good due to the formation of the horseshoe vortices. The streamline plots clearly showed that the horseshoe vortices constituted a nodal core line, along which, the particle moves in a spiral path and goes into the rear region of the tubes, which results in a good mixing and better heat transfer. Also, the heat transfer reversal (HTR) was observed behind the second row tubes. Sheu \& Tsai (1999) performed numerical simulations to determine the local conjugate heat transfer for plate and slit fins. The computational domain and numerical method was similar to the previous study (Tsai \& Sheu 1998). Most of the results were similar to the results of Tsai \& Sheu (1998). In addition, it was observed that the slit fin gives rise to the spanwise motion of the fluid particles resulting in a higher heat transfer as compared to the plate fins. However, 
the pressure drop was also higher for the slit fins as compared to the plate fins. In another study, Tsai et al (1999) performed numerical simulations to study the local conjugate heat transfer and pressure drop for the wavy slit fin. The results were almost similar to the results obtained for the plain fin. However, it was observed that the pressure difference across the wavy slit fin gives rise to the secondary flow at the transverse plane, which improves mixing and results in more heat transfer as compared to the plain fin. The pressure drop was also higher for the wavy slit fin as compared to the plain fin.

Kang \& Kim (1999) investigated the thermal-hydraulic characteristics of the strip fins and the effect of the location of the strips, and compared its performance with the plain fin. The experiments were performed on a scaled up model and a prototype model in an open wind tunnel. The scaling methods were used to compare the data obtained from the prototype and scaled up model. Four different types of fins were studied with one plain fin and three strip fins with the different configurations. It was observed that the heat transfer and pressure drop characteristics of the strip fin depends on the location of the strips. The $j / f$ factor was best for the whole plain fin (fin A), and out of all the strip fins, the fin with strips in the half rear part (fin C) was found to have the maximum $j / f$ factor (area goodness factor). Overall, it could be concluded that the fin $\mathrm{C}$ (figure 7) performs best.

Nakayama \& Xu (1983) presented the heat transfer and friction factor correlations from the test performed on the three samples of slit-finned-tube heat exchangers. However, the applicability of these was very limited. Wang et al (1999) also provided the correlations for the air-side performance of the slit-finned-tube heat exchangers. Du \& Wang (2000) conducted experiments to provide an updated correlation for the air-side performance of the slit-finned-tube heat exchangers. A total of 31 samples of slit-finned-tube heat exchangers were tested in the study with two types of slit fins. For the $1^{\text {st }}$ type of fin, the heat transfer was higher at lower fin pitch $\left(f_{p}<2.1 \mathrm{~mm}\right)$ as compared to the larger fin pitch $\left(f_{p}>2.1 \mathrm{~mm}\right)$. These results were different from the results obtained by Wang et al (1996) and for the $2^{\text {nd }}$ type of fin, the results were analogous to the results of Wang et al (1996) and the heat transfer increased with an increase in the fin pitch. After examination of $1^{\text {st }}$ type of fin, a manufacturing variation in the fin was found

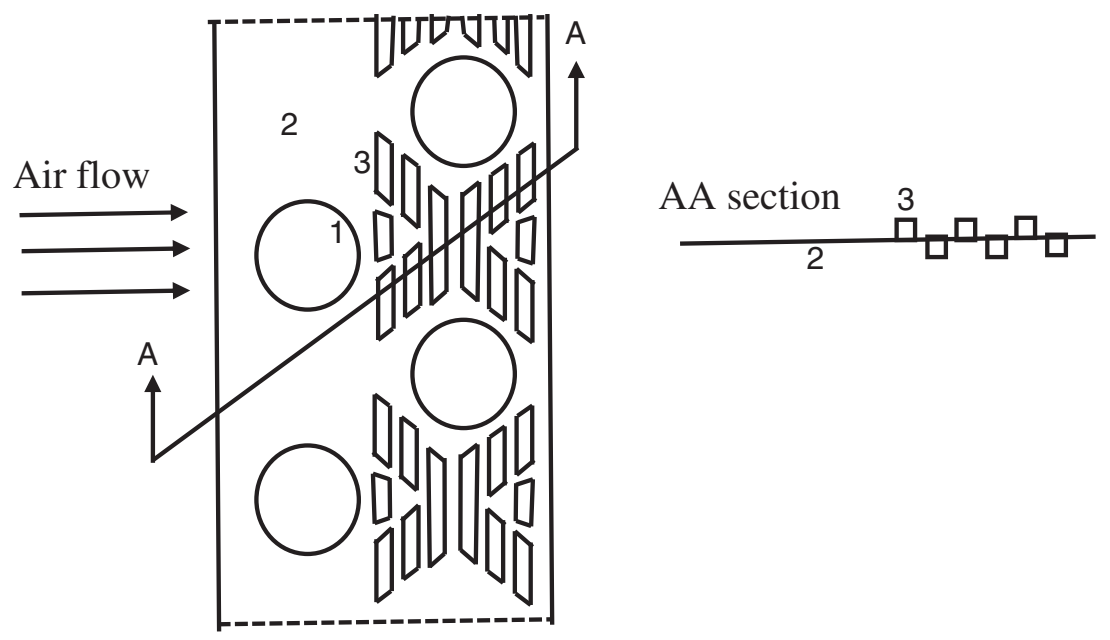

Figure 7. Strip patterns recommended by Kang \& Kim (1999), (1) tube surface, (2) plate fin surface, (3) slits. 
as compared to the fin used by Wang et al (1996) and fin of $2^{\text {nd }}$ type, which affected the mixing angle of the fins. The heat transfer performance was higher for one-row coil as compared to the multiple rows for $R e<2000$, but for $R e>2000$, the heat transfer increased for multiple row coils as compared to the one-row coil. For smaller fin pitch, the heat transfer deteriorated with an increase in the number of tube rows for $R e<1000$. Overall, the effect of the number of tube rows diminished for $R e>2000$. The reason was believed to be the increase in the turbulent intensity for $R e>2000$. A correlation was proposed for the slit-finned-tube heat exchanger air-side performance.

Yun \& Lee (2000) performed experiments to study the various parameters affecting the performance of the slit-fin heat exchanger using the Taguchi method. The experimental setup consisted of a wind tunnel with a scaled up model of the heat exchanger. Eighteen samples of heat exchangers were used for the study, and seven parameters were varied in 18 samples, e.g., fin pitch, slit height, the number of slits, slit length, the number of slit divisions, raised angle of slit, and angle of slit pattern. It was found that the effect of four factors, fin pitch (39\%), angle of slit pattern (28\%), slit length (20\%), slit height (9\%) among the seven factors was significant on the performance of the heat exchanger. The other factors affected the performance of the heat exchanger by less than $2 \%$. The optimum conditions for each of the factor were presented in the study.

Cheng et al (2004) performed numerical simulations and applied the field synergy principle (explained in Section 3.2a) to different patterns of the slotted fin surface and compared the results with the plain fin. Three types of slotted fins were used for the study and the number of strips was the same on all the fins, but the only difference was in the location of the strips. The results were validated with Wang et al (2000). It was found that all the slit fins give a higher heat transfer $(50-86 \%)$ and friction factor (36-50\%) than the plain fins. The slit fin 1 performed better than the slit fin 2 and slit fin 3, and the synergy for the slit fins was lower than the synergy for the plain fins, which indicated a higher $N u$ for the slit fins. As the $R e$ was increased, the synergy between the velocity and temperature gradient increased for all the fins. Under the same pressure drop and same pumping power conditions, slit fin 1 (figure 8) showed a better $j / f$ ratio as compared to the other fins.

$\mathrm{Qu}$ et al (2004) performed numerical simulations to determine the most effective location of the strips in the plain-finned-tube heat exchanger for a range of $348 \leq R e \leq 3480$. Four different configurations of fins were used, (A) whole plain fin, (B) strips in the upstream of the fin, (C) strips in the downstream of the fin, and (D) whole strip fin. The results showed that the

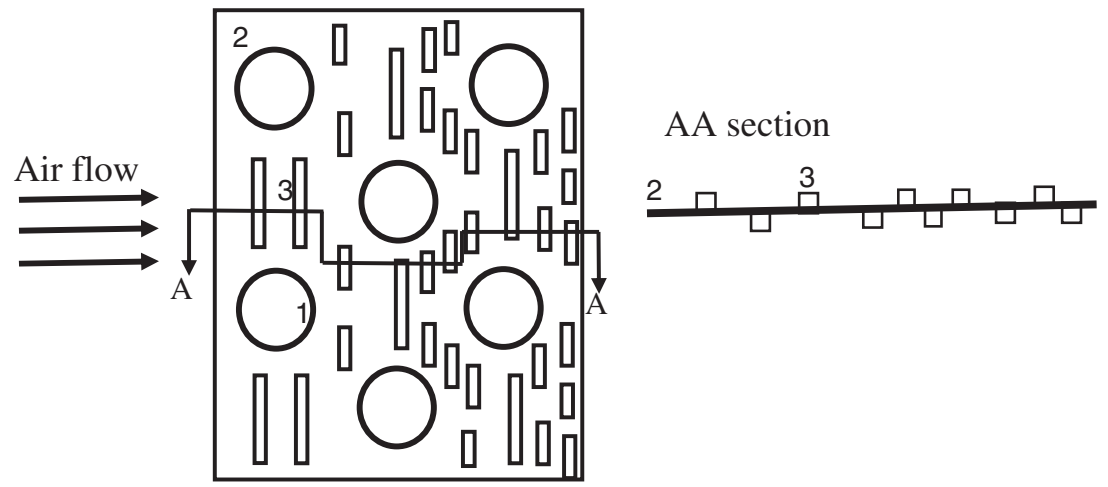

Figure 8. Strip patterns recommended by Cheng et al (2004), (1) tube surface, (2) plate fin surface, (3) slits. 
plain fin provided the least pressure drop, and out of all strip fins, fin $\mathrm{C}$ gave the least pressure drop. The fin efficiency for the fins was found to be dependent on the frontal velocity, and at low Reynolds number $(R e<1000)$, the fin efficiency was higher for the strip fins and lowest for the plain fin. However, for $R e>1000$, the fin efficiency for the plain fin became maximum and fin $\mathrm{C}$ showed the best fin efficiency out of all the strip fins. The overall heat transfer was maximum for the fin $\mathrm{D}$, but as the friction factor was also highest for the fin $\mathrm{D}$, therefore, fin $\mathrm{C}$ was recommended. All the results were explained with the help of the synergy principle as well. The synergy between the velocity and temperature gradient was minimum for fin $\mathrm{D}$, and the fin $\mathrm{C}$ showed better synergy than fin B. Based on the goodness factor, the fin C (figure 9) performed best than the others for $U_{\mathrm{fr}}<2 \mathrm{~m} / \mathrm{s}$, and above this frontal velocity, fin D performed best.

The studies presented so far discuss about the strip fin configuration in 'front coarse and rear dense'. Tao et al (2006) used numerical methods to investigate the effect of strip number, strip length, strip location, and strip distribution style on the heat transfer and pressure drop characteristics of finned-tube heat exchanger. Fifteen types of slotted fins were divided into three groups and the number of slits was varied from 3 to 6 . In the first group, the number of slits was changed, and the second group contained two different series, in the first series five slits were used with varying length, whereas, in the second series, less number of slits were used with varying lengths. The third group was made by four slits with slits cut into two. The results were presented into two different articles. The heat transfer and pressure drop characteristics results were presented in Jin et al (2006), which will be discussed next. Tao et al (2006) only presented the convergence criterion to obtain the accurate results. Three different convergence criterions were discussed, and it was found that only one of them gives consistent results. Jin et al (2006) continued the work started by Tao et al (2006), and presented the heat transfer and pressure drop characteristics data for all the slotted fins. For all the slit configurations, the $j / f$ factor was found to be greater than the $j / f$ factor for the plain fin beyond a Reynolds number value (turning $R e$ ). The decrease in the strip number resulted in a decrease in the turning Re. At low frontal velocity, the value of the $j / f$ factor decreased with an increase in the strip number. However, at higher $R e$, the effect of the number of strips was not significant. Under the identical flow rate criterion, the heat transfer for all the 15 slotted fin surfaces was higher than the plain fin. The results were

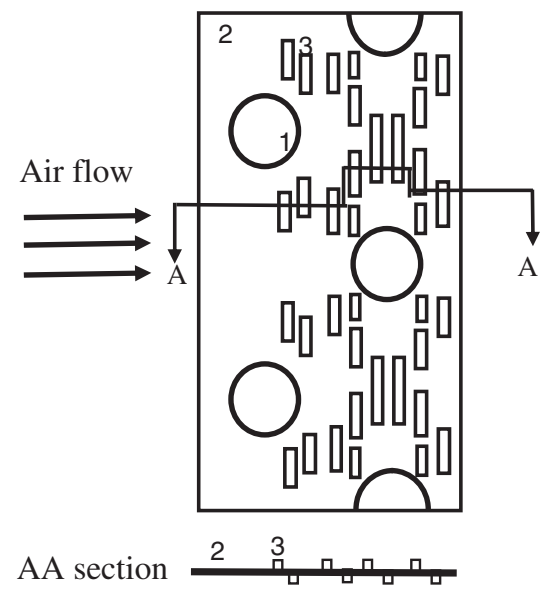

(A)

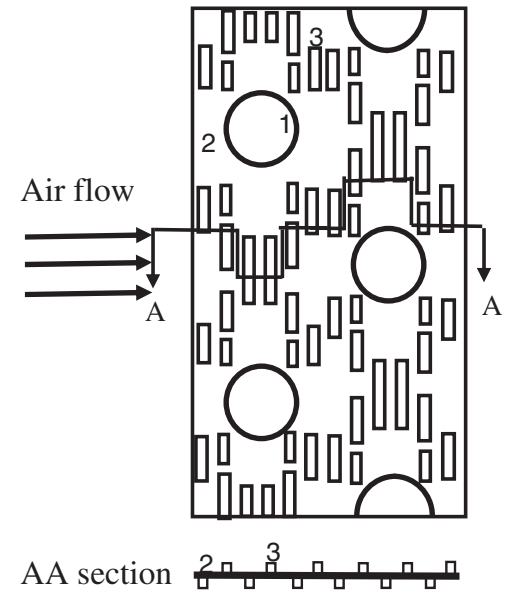

(B)

Figure 9. Slit patterns recommended by Qu et al (2004), (A) fin C, (B) fin D. [(1) tube surface, (2) plate fin surface, (3) slits]. 
also explained with the help of the synergy principle. The recommended design of the slit fins (A1-3 and slit fin 3) is shown in figure 10. The location of the slits was found to be the most prominent factor, which affects the performance of the heat exchanger, and after that strip length and strip number were the important factors.

Tao et al (2007b) performed numerical simulations to study the heat transfer and pressure drop characteristics of the slotted fins. Five different slotted fins were used for the study and the "front coarse and rear dense" principle was used in the designing of the slotted fins. Therefore, slit fin 1 had all the strips in the front part and slit fin 5 had all the strips in the rear part. The results were analyzed from the thermal resistance viewpoint. The same code was used in this study as was used by Cheng et al (2004). The slit fin 3 (figure 11) showed the highest $N u$ among all the slit fins. The fin efficiency decreased with an increase in $R e$, and it was maximum for the slit fin 3 . The thermal resistance of the slit fin 3 was equally distributed on the front and the rear portion, and it was believed to be the reason for the best performance shown by the slit fin 3 . The friction factor for the slit fin 3 was lowest for all the range of $R e$. It was recommended that the slits should be placed in such a way, that the thermal resistance of the front and the rear part becomes equal. The fin material with higher thermal conductivity improved the efficiency of the slit fins as compared to the efficiency of the plain fins.

\subsection{Studies on the comparison of fins}

In earlier sections, we have discussed the studies which focus on only one type of fin. In this section, we are going to discuss the studies in which a comparison of different types of fins is given. Yun \& Lee (1999) performed experimental investigation to study the thermal-hydraulic properties of different slit and louver type fins. The experiments were performed on a scaled

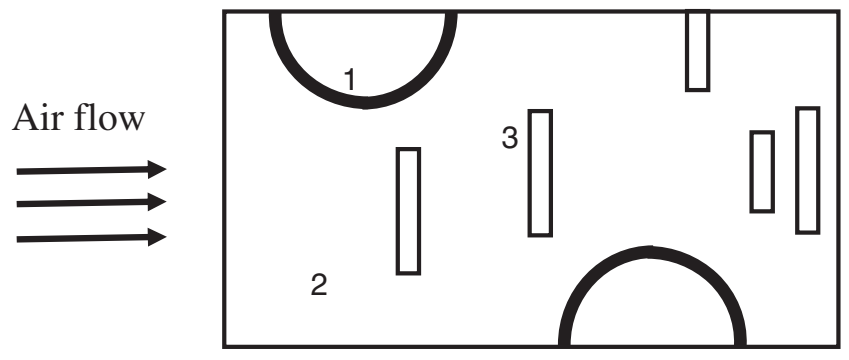

(A)

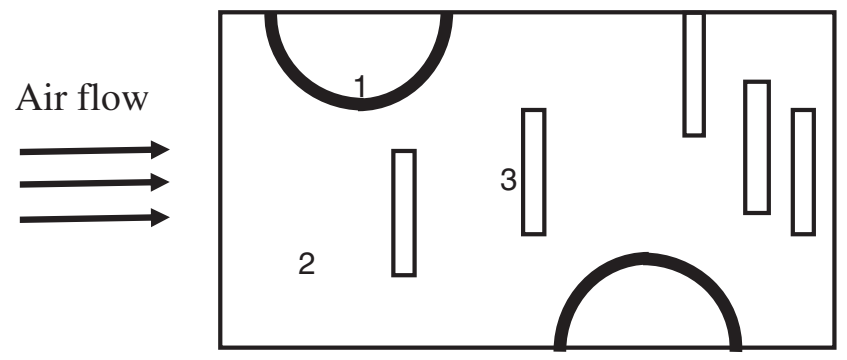

(B)

Figure 10. Slit patterns recommended by Jin et al (2006), (A) fin A1-3,(B) slit fin 3. [(1) tube surface, (2) plate fin surface, (3) slits]. 


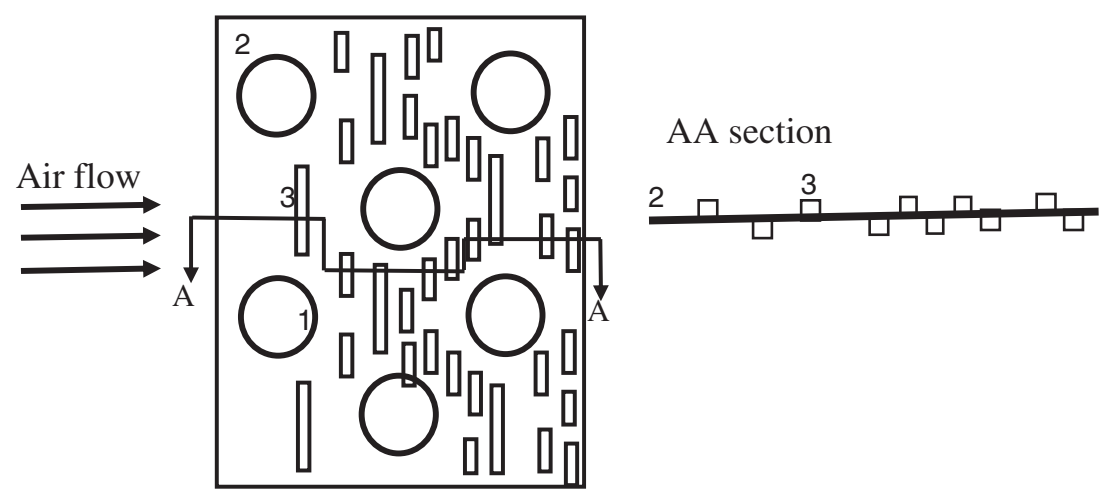

Figure 11. Slit patterns recommended by Tao et al (2007b), (1) tube surface, (2) plate fin surface, (3) slits.

up model and a prototype model in an open wind tunnel. The scaling methods were used to compare the data obtained from the prototype and scaled up model. It was observed that, for all the fin types, the error in the heat transfer data obtained from the prototype and the scaled up model was within 4.5\%; however, for the pressure drop, the error varied from 10 to $23 \%$. The effect of different slit patterns on the heat transfer coefficient was negligible; however, the effect on the pressure drop was quite significant. Yan \& Sheen (2000) performed experiments to study the thermo-flow characteristics of the plain, louver and wavy finned-tube heat exchangers. A total of 36 samples including 12 plain, 12 wavy, and 12 louver finned-tube heat exchangers were tested for a range of $300 \leq R e \leq 2000$. Both the Colburn factor and the friction factor decreased with an increase in the $R e$, and values were higher for the louver fin. The Colburn factor and friction factor increased with a decrease in the fin pitch for plain fin, but for louver fin, they did not show any trend. The heat transfer coefficient and the pressure drop increased with an increase in the frontal velocity. The effect of number of tube rows on the heat transfer coefficient was insignificant, whereas pressure drop increased with the number of rows. The area goodness factor $(\Gamma)$ was maximum for the wavy fin, and the volume goodness factor $(\gamma)$ was maximum for the louver fin. For a fixed fan power, the heat transfer was maximum and the required heat transfer area was minimum for the louver fin.

Tang et al (2009a) focused on the larger diameter tubes and studied the heat transfer and pressure drop characteristics of the finned tubes with plain fins, slit fins, and fins with DWP. Nine samples of heat exchangers were tested in a wind tunnel with three types of fins and varying number of rows $\left(6 \leq N_{r} \leq 12\right)$. $N u$ and the friction factor were found to be independent of the number of rows for all the three types of fins. At the same frontal velocity, $N u$ and the friction factor for the slit fin were higher than the plain fin and the fin with DWP, and the friction factor for the fin with DWP and the plain fin was almost equal. Under the same mass flow rate criterion, same pressure drop criterion, and same pumping rate criterion, at low $R e$, the slit fin showed highest $\mathrm{Nu}$; however, the pressure drop was also highest. As $R e$ was increased, the $j / f$ ratio increased for the slit fin at a higher rate as compared to the other two fins. Therefore, it was concluded that the slit fin performs better, and the fin with the DWP must be designed carefully. Tang et al (2009b) extended their previous study to crimped spiral fins, plain fins, slit fins, fin with DWP, and mixed fins (DWPs in front and slits in rear). The experimental setup 
was validated with the results of ZukausKas (1972) and Jakob's (1938) correlation. The Genetic algorithm optimization was used for the optimization of the fin with the DWP. The numerical simulations were also performed for the fin with DWP to optimize the attack angle, length and height of the DWP. It was observed that $N u$ and pressure drop increase with an increase in the frontal velocity, and for the same frontal velocity, the crimped spiral fin provided highest $\mathrm{Nu}$, and slit fin showed the second highest. However, the pressure drop was also highest for the crimped spiral fin. At the identical mass flow rate criterion, identical pumping power criterion, and the identical pressure drop criterion, fin with DWP showed the highest $N u$ for $R e<4500,4500$, and 5000 , respectively. However, for $R e>4500,6000$, and 6700, for identical mass flow criterion, identical pumping power criterion, and identical pressure drop criterion, respectively, the slit fin performed best. After optimization, it was found that the DWP could perform better than the slit fin. The correlations for the Colburn factor and the friction factor were developed for the fin with DWP.

\section{Effects of different parameters}

\subsection{Reynolds number}

The Reynolds number is the most common parameter which has been studied by various researchers. However, the basis for the calculation of Reynolds number has been divided mainly into three categories. First is the tube outer diameter or tube collar diameter, second is the hydraulic diameter of the finned tubes, and third is the fin spacing. We have discussed the basis for the calculation of Reynolds number in detail in Section 2. The heat transfer performance of the heat exchangers depends on the behavior of the boundary layer, formation of vortices and eddies, and generation of turbulence. For the plain fin, at low Reynolds number $\left(R e_{\mathrm{h}}=211\right)$, the enhancement in the heat or mass transfer is due to the boundary layer growth (Saboya \& Sparrow 1976), and at higher Reynolds number the enhancement is mainly due to the formation of the vortices. The effect of the other geometrical parameters like fin pitch and number of tube rows depends on the value of the Reynolds number, and from the literature, it has been observed that the effect of these geometrical parameters diminishes above a $R e>2000$. For the fins with DWP, the formation of the longitudinal and transverse vortices is important, and with the increase in the Reynolds number, these vortices get stronger. The increment in the heat transfer with an increase in Reynolds number has been found to be higher for the fins with DWP as compared to the plain fins (Tiggelbeck et al 1994). The geometrical configuration of the winglet affects the transitional Reynolds number value and the formation of the vortices. For wavy-finnedtube, Wang et al (1996) found that the downstream turbulence shedding starts at $R e=900$ for staggered arrangement, and at $R e=2000$ for the inline arrangement. These results could be extended to the other type of finned-tubes as well. For the annular fin, the effect of the Reynolds number has been found similar to the effect of Reynolds number on the plain fin. For the offstrip fins (Jin et al 2006), the effect of the strip number was significant on the heat transfer and pressure drop; however, at high Reynolds number $(R e>2250)$, the effect of the strip number diminished, and all the four slotted fins performed equally well.

On the basis of the foregoing discussion, we can conclude that, beyond a value of Reynolds number the effect of the other geometrical parameters (example: fin pitch, number of tube rows, and number of strips in strip fin) on the heat transfer coefficient and friction factor tends to diminish. The main reasons being the downstream vortex shedding and turbulence generated at

higher Reynolds number $(R e>5000)$. 


\subsection{Fin pitch}

The fin pitch is the second most important parameter which affects the performance of the finnedtube heat exchangers. In Section 4.1, we discussed the importance of the boundary layer growth at the fin surface and the formation of the horseshoe vortices at the tube surface with the variation in $R e$. The fin pitch affects both of these physical phenomena. At a constant $R e$, as the fin pitch is varied, the interaction between the boundary layers on the two fin surfaces gets affected, which results in a variation in the heat transfer. The effect of fin pitch on the heat transfer and pressure drop is mainly dependent on three other parameters, Reynolds number, number of tube rows and condensate formation on the heat transfer surface (for wet surface conditions). The effect of fin pitch is also different for different fins. For the plain fin, Wang et al (1996) did not observe any significant effect of the fin pitch on the Colburn factor and the friction factor but in their another study (Wang \& Chi 2000) found that for one and two rows coils, the heat transfer increases with a decrease in the fin pitch $\left(1.19 \mathrm{~mm} \leq f_{\mathrm{p}} \leq 3.31 \mathrm{~mm}\right)$ for a $R e<5000$. Similarly, results were obtained by Yan \& Sheen (2000) for heat transfer for a fin pitch of 1.4$2 \mathrm{~mm}$; however, the friction factor was found to increase with a decrease in the fin pitch. Chen et al (2005), Huang et al (2009) and Choi et al (2010) varied the fin pitch in a larger range (5 $\mathrm{mm} \leq f_{\mathrm{p}} \leq 30 \mathrm{~mm}$ ), and found that the heat transfer coefficient increases with an increase in the fin pitch. Wang \& Chi (2000) argued that, at lower fin pitch, the flow can be kept as laminar and vortex behind the tube is suppressed; however, they did not explain the effect of the boundary layer interaction between the fins and horseshoe vortices on the heat transfer. Choi et al (2010) attributed the increase in the heat transfer with an increase in the fin spacing to the delay in the boundary layer interaction at larger fin spacing. Romero-Méndez et al (1997), He et al (2005) and Liu et al (2010) observed that the heat transfer coefficient increases up to a value of the fin pitch, and after that, it decreases. The reason was again the delay in the boundary layer interaction at larger fin pitch, however, beyond a certain value of the fin pitch, the flow bypassed the finned surface area, and heat transfer between the fins was inefficient. The maxima was found at $S / D=0.167$ (tube OD not given) by Romero-Mendez, at $S / D=0.06(D=10 \mathrm{~mm})$ by $\mathrm{He}$ et al, and at $S / D=0.152(D=16.68 \mathrm{~mm})$ by Liu et al. The occurrence of the maxima at different $S / D$ ratios can be caused by the different values of the tube diameter, which affects the size of the horseshoe vortices and ineffective area behind the tube. In the dehumidifying conditions, the effect of fin pitch on the heat transfer was negligible due to the turbulence generated by the condensate. In all the studies for plain fin, the friction factor or pressure drop decreased with an increase in the fin pitch except for Liu et al, where the pressure drop increased with an increase in the fin pitch due to more accumulation of condensate at larger fin pitch. For wavy fin, Wang et al (1997) and Wongwises \& Chokeman (2005) did not find any significant effect of the fin pitch on the heat transfer. At lower Reynolds number $(R e<4000)$, Pirompugd et al (2005, 2008), and Cheng et al (2009) found that the heat transfer was higher for the smaller fin pitch $\left(1.2 \leq f_{\mathrm{p}} \leq 3.5\right)$, and the reason was believed to be the laminar flow and suppressing of the vortex region behind the tube at smaller fin pitch [similar to the Wang \& Chi (2000)]. Tao et al (2011a) found a maxima in the heat transfer for a fin pitch of $1.2 \leq f_{\mathrm{p}} \leq 2 \mathrm{~mm}$. The results were analogues to the other authors as Tao et al kept the Reynolds number below 4000 . The friction factor was found to be higher for the smaller fin spacing in all the studies. For annular fin, Watel et al (2000a, b) and Chen \& Hsu (2008) found that $N u$ increases with an increase in the fin pitch $\left(2 \leq f_{\mathrm{p}} \leq 40 \mathrm{~mm}\right)$. Mon \& Gross (2004) found a maxima in the $N u$ at $S / D=0.0875$, and they concluded that, with an increase in the fin pitch, the horseshoe vortices get stronger and the thermal boundary layer becomes thinner resulting in an increase in the heat transfer. Pongsoi et al (2013) found that, the Colburn factor was independent of the fin pitch $\left(2.4 \mathrm{~mm} \leq f_{\mathrm{p}} \leq 4.2\right.$ 
$\mathrm{mm}$ ) for all Reynolds number considered in the study; however, the friction factor increased as the fin pitch was increased for $R e>6000$, and for $R e<6000$, the fin pitch did not affect the friction factor. For the crimped spiral fin, Kawaguchi et al (2004) found that the effect of the fin pitch depends on the transverse tube pitch and bypass flow rate. For inline arrangement, at higher tube pitch $\left(S_{\mathrm{t}}=71.4 \mathrm{~mm}\right)$, the increment in the fin pitch did not affect the pressure drop as flow bypassed the finned region. However, for a smaller tube pitch $\left(S_{\mathrm{t}}=50 \mathrm{~mm}\right)$, the pressure drop increased with a decrease in the fin pitch. The heat transfer in both the cases increased with an increase in the fin pitch and the reason was thought to be the decrease in the bypass flow rate with an increase in the fin pitch, however, the role of the horseshoe vortices was not explained in the study. Similarly, for the staggered arrangement at higher transverse tube pitch, the heat transfer decreased at lower fin pitch due to bypassing of the flow. For lower tube pitch, the heat transfer was independent of the fin pitch, as the phenomenon of bypass flow was not so significant. Pongsoi et al (2011, 2012b) found that the heat transfer becomes independent of the fin pitch for a transverse tube pitch of $40 \mathrm{~mm}$, and the reason was attributed to the high Reynolds number $(4000 \leq R e \leq 13000)$. However, an optimum fin pitch of $4.2 \mathrm{~mm}$ was suggested. Kawaguchi et al (2004) observed that the friction factor for serrated fins was 1.15 times the friction factor for the annular fins at $f_{\mathrm{p}}=5 \mathrm{~mm}$, and it reduced to 1.1 times at $f_{\mathrm{p}}=3.3 \mathrm{~mm}$. This shows that the increase in the friction factor with the fin pitch was larger for the plain annular fins. It was believed that the turbulence generated by the segmentations increases the friction factor for the serrated fins. However, smaller force requirement for changing the flow direction across the fins for serrated fins and entrainment of the flow in the wake region due to the turbulence generated by the serrated fins were believed to be the two factors, which limited the friction factor for the serrated fins. The effect of fin pitch on the heat transfer was found to be negligible for serrated fins. Ma et al (2012) observed that $\mathrm{Nu}$ decreased in the range of 11 to $0 \%$ as the fin pitch was decreased from $4.2 \mathrm{~mm}$ to $3.9 \mathrm{~mm}$ in the Reynolds number range of $4000 \leq R e \leq 30000$. A critical Reynolds number was found, above which the effect of $h_{\mathrm{f}} / S$ ratio on the heat transfer was negligible. The Euler number was increased by $8 \%$, when the fin pitch was decreased from $4.2 \mathrm{~mm}$ to $3.9 \mathrm{~mm}$. For slit fins, Yun \& Lee (2000) proposed an optimized fin pitch of $3.6 \mathrm{~mm}$.

From all these previous studies, it can be concluded that the effect of fin pitch depends on the fin type. However, most of the authors have obtained maxima in the heat transfer for a particular fin pitch, and this optimum value of fin pitch depends on the other parameters like tube diameter, wet or dry surface conditions and Reynolds number. This optimum value lies between 1.2 and $4 \mathrm{~mm}$ for a $R e>3000$ for almost all types of finned-tubes with $D>8 \mathrm{~mm}$. However, some of the authors have obtained the heat transfer coefficient as an increasing or a decreasing function of the fin pitch. Therefore, further studies are required to capture the flow physics and effect of the fin pitch on the thermal-hydraulic performance of the finned-tube-heat exchangers. Furthermore, in the dehumidifying conditions, the effect of the fin pitch varies due to the presence of the condensate. Therefore, in the dehumidifying conditions, the fin pitch must be optimized carefully.

\subsection{Effect of fin thickness}

For plain fin, Wang et al (1996) studied the effect of the fin thickness, and observed that it does not affect the heat transfer or pressure drop. Madi et al (1998) found that fin with a thickness of $0.12 \mathrm{~mm}$ performed better than the fin with thickness of $0.13 \mathrm{~mm}$. For wavy fin, Wongwises \& Chokeman (2005) found that the effect of fin thickness depends upon the number of tube rows. For 2-row coil, as the fin thickness was increased from $0.115 \mathrm{~mm}$ to 0.250 
$\mathrm{mm}$, then the horseshoe vortices became stronger, and the heat transfer enhancement due to the horseshoe vortices dominated over the heat transfer decrement due to the wake region behind the tubes. However, for 4-row coil, the wake region behind the tubes dominated over the formation of horseshoe vortices, and the Colburn factor decreased with an increase in the fin thickness at $R e<1800$. For $R e>1800$, the horseshoe vortices became stronger than the wake region behind the tubes. The friction factor also increased with an increase in the fin thickness. Tao et al (2011) varied the fin thickness in the range of $0.05 \leq t_{\mathrm{f}} \leq 0.42 \mathrm{~mm}$, and they observed that $\mathrm{Nu}$ and friction factor both increase with an increase in the fin thickness. This was due to the consideration of same frontal velocity for all the cases, due to which, at larger fin thickness, the fin spacing decreased and the maximum velocity between the fins increased, resulting in an increase in the heat transfer and pressure drop. For fins with delta winglet, Zeng et al (2010) observed that the effect of the fin thickness was negligible and hence was neglected. However, similar to the case of Tao et al (2011), the effect of the fin thickness was observed at the same frontal velocity and a fin thickness of $0.1-0.12 \mathrm{~mm}$ was suggested. In wet conditions, Kuvannarat et al (2006) observed that, for 2-row coil and small fin pitch $(1.41 \mathrm{~mm})$, the heat transfer coefficient for $t_{\mathrm{f}}=0.25 \mathrm{~mm}$ was $5-50 \%$ higher than for $t_{\mathrm{f}}=0.115 \mathrm{~mm}$, and the corresponding pressure drop was 5-20\% higher for $t_{\mathrm{f}}=0.25 \mathrm{~mm}$. At lower fin spacing, the droplet size was found to be comparable to the fin spacing, and it produced swirling motion and vortices which helped in better mixing in the main flow. However, at higher fin spacing, the mixing was not pronounced and the effect of the fin thickness was negligible. At larger number of tube rows $\left(N_{\mathrm{r}}=6\right)$, the effect of the fin thickness and spacing reduced and became negligible.

From this section, we can conclude that at a constant frontal velocity, when the fin thickness is varied, then it results in an increase in the maximum velocity, which enhances the heat transfer and pressure drop. An optimized fin thickness of $0.1-0.2 \mathrm{~mm}$ can be suggested from this discussion.

\subsection{Effect of fin height}

The increment in the fin height increases the heat transfer area, due to this the heat transfer rate gets enhanced; however, the pressure drop also increases due to more friction and blockage provided to the flow. The effect of fin height on the heat transfer coefficient, however, depends on the type of fin and dry or wet conditions. For crimped spiral fins, Nuntaphan et al (2005a, b) found that for the inline arrangement, increase in the fin height from 10-15 mm results in an increase in the pressure drop up to $100 \%$ and it decreased the heat transfer coefficient by $50 \%$ at lower frontal velocity $(0.7 \mathrm{~m} / \mathrm{s})$ and by $90 \%$ for $U_{\mathrm{fr}}=1.5 \mathrm{~m} / \mathrm{s}$. This was believed that the flow bypasses the high resistive fin regions (for larger diameter fin) and do not participate in the heat transfer. For staggered arrangement, the effect of fin height was not significant on the pressure drop, because most of the pressure drop was caused by the staggered arrangement of the tubes. In the similar study in wet conditions Nuntaphan et al (2005b) found that the condensate resistance dominates over the effect of fin height on the heat transfer coefficient resulting in a negligible effect of the fin height on heat transfer coefficient, whereas the pressure drop increased with an increase in the fin height. For serrated fin, Næss (2010) observed that the heat transfer coefficient increases with an increase in the fin height $\left(8.61 \leq h_{\mathrm{f}} \leq 11.38 \mathrm{~mm}\right)$, whereas the effect of fin height on the pressure drop was found to be negligible. This result was found to be different from the prediction by other correlations and it was thought that the earlier correlations were based on the performance of the assumption of similar behavior of serrated fin and plain annular. However, this assumption does not hold true, because the fluid may not penetrate as efficiently to 
the fin root of plain annular fin as to the fin root of the serrated fin, which results in more mixing for the serrated finned tubes. Therefore, the correlations should be developed on the basis of the performance of the serrated fin.

Overall, it can be concluded that the increase in fin height results in an increase in the average heat transfer and pressure drop. However, the heat transfer coefficient decreases with an increase in the fin diameter beyond a certain value. Therefore, fin height should be optimized to obtain the maximum heat transfer at the lowest total cost of the heat exchanger, and for that purpose, the capital cost of the heat exchanger, the area goodness factor and the volume goodness factor must be considered. For annular fins, a fin height of 5-10 mm can be suggested for better performance.

\subsection{Effect of tube diameter}

For plain fin, Wang \& Chi (2000) observed that the heat transfer coefficient was higher for lower tube diameter $(D=8.5 \mathrm{~mm})$ as compared to the larger tube diameter $(D=10.23 \mathrm{~mm})$ due to the increase in the ineffective area behind the tubes for larger diameter tubes. For 1-row coil, the heat transfer coefficient was higher for the larger tube diameter (as the increase in the ineffective area for 1-row was smaller). The total heat transfer rate and pressure drop were higher for the larger tube diameter. Similar results for plain fin were obtained by Xie et al (2009). For crimped spiral fin, Nuntaphan et al (2005a, b) found similar results to those obtained by Wang \& Chi (2000) for the plain fin. Cheng et al (2009) presented the results in terms of $N u$ and pressure drop for the wavy fin and observed that $\mathrm{Nu}$ was $21 \%$ higher for $D=11.2 \mathrm{~mm}$ and $33 \%$ higher for $D=13.6 \mathrm{~mm}$ as compared to that for $D=8.8 \mathrm{~mm}$. The friction factor was found to be $33 \%$ higher for $D=11.2 \mathrm{~mm}$ and $83 \%$ higher for $D=13.6 \mathrm{~mm}$ as compared to that for $D=8.8$ $\mathrm{mm}$. The high $N u$ did not represent the high heat transfer, as the heat transfer coefficient is a ratio of $\mathrm{Nu}$ to the diameter of the tube, hence the heat transfer coefficient was higher for the tube with $\mathrm{D}=8.8 \mathrm{~mm}$.

From all of these studies, it can be concluded that the increase in the tube diameter results in a decrease in the heat transfer coefficient and increase in the pressure drop irrespective of the fin type. Therefore, the use of smaller tubes with $O D$ ranging from 7 to $10 \mathrm{~mm}$ should be preferred. However, as we decreases the tube diameter, the pressure drop on the tube side gets enhanced, therefore, that part should be taken into consideration while designing the air-cooled heat exchangers.

\subsection{Tube pitch}

The variation in the transverse tube pitch results in a variation in the flow area between the tubes. The effect on the heat transfer and pressure drop depends on whether the system is operated at a constant frontal velocity or at a constant Reynolds number (based on the maximum velocity). For plain fin, He et al (2005) and Xie et al (2009) observed that, for a fixed inlet velocity, the heat transfer and pressure drop decrease with an increase in the transverse tube pitch. For serrated fins, Kawaguchi et al (2004) found that the heat transfer and pressure drop were independent of the tube pitch (40 mm $\leq S_{\mathrm{t}} \leq 45 \mathrm{~mm}, 30 \mathrm{~mm} \leq S_{1} \leq 40 \mathrm{~mm}$ ). Næss (2010) observed that the transverse tube pitch had significant effect on the heat transfer for $S_{\mathrm{t}} / D_{\mathrm{f}}=2$. Ma et al (2012) found that the transverse tube pitch has negligible effects on the heat transfer (less than 3\%), whereas the heat transfer gets affected by the longitudinal tube pitch. Furthermore, they observed that the heat transfer remains independent of the transverse tube pitch for a tube pitch to fin diameter ratio $\left(1.2 \leq S_{\mathrm{t}} / D_{\mathrm{f}} \leq 1.7\right)$ for their study and for $1.2 \leq S_{\mathrm{t}} / D_{\mathrm{f}} \leq 1.5$ for Kawaguchi et al (2004). It was concluded that there should be an optimum transverse to longitudinal pitch ratio 
for a specific transverse tube pitch. $E u$ was found to decrease by $20 \%$ with an increase in the transverse tube pitch from $88 \mathrm{~mm}$ to $120 \mathrm{~mm}$, whereas the effect of the longitudinal tube pitch on the Euler number was negligible.

It can be concluded that the effect of transverse tube pitch depends on the fin type. For serrated fins, it affects the heat transfer results above a certain value of $S_{\mathrm{t}} / D_{\mathrm{f}}$ ratio $(0.2)$. For plain fin, the heat transfer and pressure drop decrease with an increase in the transverse tube pitch; however, for the better performance, the transverse to longitudinal pitch ratio should be optimized.

\subsection{Tube type}

The flow separation at the tube surface and the formation of the wake region is very prominent for the circular tubes. It results in an ineffective area behind the tube, which in turn decreases the heat transfer coefficient. The form drag for the circular tubes is also high and results in a larger pressure drop as compared to the elliptical and oval tubes. The elliptical and oval tubes have less ineffective area behind the tubes and lesser form drag. Various studies have been carried out to compare the performance of the circular and elliptical tubes (or oval tubes). For plain finned tubes, Rocha et al (1997) found that maximum fin efficiency is obtained with an ellipticity (e) of 0.5. Saboya \& Saboya (2001) recommended the elliptical tube, as it provided a better fin efficiency. Erek et al (2005) observed that the elliptical tubes give more heat transfer coefficient and lesser pressure drop as compared to the circular tubes. Ibrahim \& Gomaa (2009) studied the elliptical tubes at different attack angles with respect to the incoming air flow. They found that at an angle of attack of $0^{\circ}$, the heat transfer per unit pressure drop, area goodness factor and efficiency index were maximum. The average $N u$ was larger for the larger angle of attack and was maximum for $90^{\circ}$, and was $19 \%$ greater than the circular tubes. However, the friction factor was also increased with an increase in the angle of attack, and it was maximum at $90^{\circ}$, and was $65 \%$ greater than the circular tubes. For annular fins, Jang \& Yang (1998) observed that the heat transfer per unit pressure drop was $50 \%$ higher for the elliptical tubes as compared to the circular tubes. Fiebig et al (1994) put DWP with the round and the flat tubes, and observed that the circular tubes perform better than the flat tubes without DWP, but the flat tubes with DWP gave two times the heat transfer and half the friction factor as compared to the round tubes with DWP. The physical reason behind this was believed to be the absence of the horseshoe vortices for the flat tubes without DWP, because the flat tubes were placed near the fin edge, and hence heat transfer was less for the flat tubes as compared to the round tubes. As the DWP was put, they generated vortices and $N u$ for the flat tubes with DWP became higher as compared to the $\mathrm{Nu}$ for the round tubes with DWPs.

Overall, it can be concluded that the heat transfer per unit pressure drop is always higher for the elliptical and flat tubes as compared to the circular tubes and they are recommended for the practical purpose. However, the area goodness factor and the volume goodness factor should also be optimized for an economical design.

\subsection{Number of tube rows}

The effect of number of tube rows depends mainly on the tube arrangement, value of the Reynolds number and wet or dry conditions. For plain fin, Wang et al (1996) and Wang \& Chi (2000) found that the heat transfer coefficient decreases with an increase in the number of tube rows (maximum 6) for $R e<3000$, and the effect of tube rows on the friction factor was found to be negligible. Beyond $R e>3000$, the effect of number of tube rows diminished due 
to better mixing at high Re. He et al (2005) recommended a maximum 3 rows of the tubes for the practical purpose. Xie et al (2009) and Choi et al (2010) found that both the heat transfer coefficient and friction factor decrease with an increase in the number of tubes rows, and for $N_{\mathrm{r}}>6$, the effect of tube rows diminishes. Liu et al (2010) studied plain fin in wet conditions and observed that the effect of tube rows depends on the fin pitch and Reynolds number. For $R e>4000$, the Colburn factor was found to decrease with an increase in the number of tube rows $\left(2 \leq N_{\mathrm{r}} \leq 8\right)$. They argued that this was associated with the condensate blow off phenomenon (at lower Reynolds number the condensate is more prone to adhere to the surface of the fin, and it provides more mixing in the flow which makes the effect of tube rows negligible). At larger fin spacing also the effect of tube row number diminished because large condensate was prone to suspending between fins at larger fin spacing. For wavy fin, Wang et al (1997) found that, for the staggered arrangement, the heat transfer coefficient decreases with an increase in the row number for $R e<900$, and beyond that a slight increase in the heat transfer coefficient was observed with an increase in the row number. For the inline arrangement, the heat transfer coefficient decreased with an increase in the row number for $R e<2000$, and above this $R e$, the effect of tube rows diminished. Wongwises \& Chokeman (2005) observed that the Colburn effect and friction factor decrease with an increase in the row number for $R e<4000$, and for $R e>4000$, no effect of tube row number on the Colburn factor and friction factor was observed. Similar results were obtained for the slit fin by Du \& Wang (2000), and the effect of tube rows on the heat transfer and pressure drop performance became negligible for $R e>2000$. Tang et al (2009a) studied slit fin, plain fin and fin with DWPs and observed that the heat transfer coefficient and friction factor were independent of number of tube rows $\left(6 \leq N_{\mathrm{r}} \leq 12\right)$. For fin with DWPs, Kwak et al (2003) varied the tube rows in the range of $2 \leq N_{\mathrm{r}} \leq 5$, and observed that the Colburn factor was maximum for 2 rows and decreased as the row number was increased. The friction factor was minimum for 3 rows and beyond $R e>1000$, the effect of row number on the friction factor diminished. Overall, the three-row coil performed best with a 30-10\% augmentation in the heat transfer and a reduction of 55-34\% in the pressure drop for $350 \leq R e \leq 2100$. The lower pressure drop penalty for 3-row coil was explained as: The flow gets accelerated between the DWP and the tube surface, and it reduces the wake region for downstream rows and brings separation delay. The form drag reduces per unit length as we go downstream. However, the effect of DWP does not reach up to 4 and 5 rows and hence the pressure drop increases for 4 and 5 row coils.

Overall, it can be concluded that a row number of 3 has been recommended by various authors and the effect of row number diminishes for $N_{\mathrm{r}}>6$, and $\operatorname{Re}>3000$.

\subsection{Effect of dehumidifying conditions}

There are two major effects of water condensate, one is the turbulence generated by the droplets which enhances the heat transfer and the other is the water film resistance which degrades the heat transfer. The effect of water condensate also depends on other parameters, for example above a certain value of Reynolds number, the turbulence becomes dominant over the film resistance. Along with the effects on the heat transfer, the condensate affects the pressure drop as well, by providing more resistance to the flow. The turbulence generated by the condensate also influences the effect of other geometrical parameters on the heat transfer and pressure drop. For plain fin, Pirompugd et al (2005) observed that the effect of fin spacing on the heat transfer diminishes because of the presence of the condensate, which enhances the mixing by roughening the surface. The effect of number of tube rows was also affected by the condensate. Pirompugd et al $(2007 \mathrm{a}, \mathrm{b})$ observed that the fin efficiency obtained for the partial wet surface was higher 
than the efficiency for the fully wet surface and was lower than the efficiency for the fully dry surface. The fin efficiency decreased with an increase in the relative humidity. For wavy fin, Wang et al (2000) found that the pressure drop in wet conditions was higher for the wavy fins due to the generation of the swirling flow behind the droplets. This effect was more prominent for larger waffle height and smaller fin spacing. Pirompugd et al (2006) observed that, at lower fin spacing, the increase in the inlet humidity gives rise to a lower mass transfer because of the condensate retention phenomenon; however, if Reynolds number is increased above 1000 (at inlet humidity of 50\%), then the mass transfer increases because of the blow-off of condensate by the flow inertia. Kuvannarat et al (2006) observed that at higher fin pitch $\left(f_{\mathrm{p}}=2.54 \mathrm{~mm}\right)$, the effect of water condensate mixing was not significant.

Therefore, it can be concluded that the presence of the water condensate at low Reynolds number degrades the heat transfer because of the thermal resistance provided by the condensate, however, as the Reynolds number is increased $(R e>1000)$ then the swirling motion provided by the condensate helps in mixing in the flow and it improves the heat transfer. The presence of condensate always leads to more pressure drop.

\section{Conclusions and recommendation for future work}

(i) From all the studies discussed in this review, it may be noted that most of the studies focus on the thermal-hydraulic performance of the heat exchangers. However, none of the studies have focused on the optimization of the heat exchangers with respect to the cost of the heat exchanger. The capital cost of the condenser can be optimized by maximizing the heat transfer coefficient, and hence minimizing the heat transfer area. However, the associated pressure drop must be minimized for obtaining a minimum operating cost. Various studies have discussed the optimization of these factors; however, the real cost of the heat exchangers have not been discussed in these studies. The other costs include the cost associated with the space required, for that one need to design a very compact heat exchanger. For this purpose, the area goodness factor and volume goodness factor have to be optimized; however, only few studies have focused on the optimization of these factors, and in these studies also, the cost associated with the required space have not been discussed.

(ii) A review has been presented on the thermal-hydraulic performance of the air-cooled heat exchangers. The experimental studies have been performed by many researchers for the last 50-60 years. The experimental studies mostly lack in determining the 3D flow patterns, temperature contours and velocity vectors.

(iii) A great improvement in the numerical methodologies and computational capability has led us to understand the 3D flow patterns around the finned tubes. However, some of the fins are very complex to model (example: serrated fin, crimped spiral fins), and the numerical studies on these fins have been very limited. Therefore, more numerical studies are needed for these types of fins. Furthermore, in all the studies only two or three fins are taken in the computational domain and a periodic boundary condition is assumed to model the whole length of the tube. However, it has been observed that by varying the fin pitch along the length of the tube, the thermal-hydraulic performance can be improved. Therefore, more of 3D numerical studies should be performed to model the whole length of the tube by varying the fin spacing along the length.

(iv) From the discussion in Sections 3 and 4, it is clear that the estimation of heat loss in the published literature has been addressed using two types of approaches: (1) development of empirical correlations and (2) use of CFD. The latter approach permits the understanding 
of physics of the system through the insights in (a) fluid mechanics and (b) the relationship between the fluid mechanics and design objectives such as heat losses. Secondly, during the past 25 years, CFD is being increasingly used because of the development in computational power as well as numerical techniques. Joshi \& Ranade (2003) have given an overview of opportunities and scope of CFD. Ranade et al (1989; Ranade \& Joshi 1990, 1992), Murthy \& Joshi (2008), Ekambara et al (2005), and Joshi et al (2011a, b) have given the details pertaining to governing equation, method of solution and appropriate precautions for the implementation of CFD. Furthermore, some examples of relationship between the fluid mechanics and design objectives have been described in the published literature. For instance, heat transfer (Thakre \& Joshi 1999), mixing (Patwardhan \& Joshi 1999; Nere et al 2003; Kumaresan \& Joshi 2006; Joshi \& Sharma 1978; Joshi \& Shah 1981), solid suspension (Raghav Rao et al 1988; Rewatkar \& Joshi 1991; Murthy et al 2007b) and the rate of gas induction (Murthy et al 2007a; Joshi \& Sharma 1977). Similarly, methodology needs to be employed in the future work for the estimation of heat losses and pressure drop. In particular, LES (and if possible DNS) simulations need to be undertaken for developing better insight.

(v) For better understanding of transport phenomenon, the future work should include the identification and dynamics of flow structures. (Joshi \& Sharma 1976; Thorat et al 1998; Kulkarni et al 2001; Bhole et al 2008; Joshi et al 2009; Mathpati et al 2009). Additional work is also needed to understand the relationship between the structure dynamics and heat transfer as well as pressure drop.

(vi) For crimped spiral fins, all the studies have been experimental and empirical correlations have been developed. The visualization of flow pattern, development and breaking of the boundary layer has been missing from the literature. Therefore, it is recommended to perform numerical simulation to understand the flow physics for the crimped spiral-finned-tube heat exchangers.

(vii) For serrated fins as well, the flow physics has not been investigated extensively and only one numerical study by Lemouedda et al (2011) has been reported. In that study also, the pressure drop across the finned-tube heat exchanger was not presented; therefore, the performance of the serrated fins as compared to the plain annular fin could not be determined. Therefore, most of the numerical studies are recommended in this case also.

(viii) Banerjee et al (2012) modified the annular fin into perforated fins and they obtained excellent results for the perforations in the wake region. These types of fins enhance the heat transfer in the wake region; however, only one study is available on this kind of fin. Therefore, more studies should be performed in order to optimize these kinds of fins for commercial use.

(ix) Banerjee et al (2012) performed 2D numerical study, in which they varied the fin pitch along the length of the finned tube, and they observed a reduction in the pressure drop. However, the effect on the heat transfer was unknown. Therefore, it is recommended to perform 3D numerical study by varying the fin spacing along the length of the tube to determine the thermal-hydraulic performance of the heat exchangers.

(x) For the slit fins, most of the studies are performed with the circular tubes, further studies can be performed to compare the performance of slit fins with the Elliptical or flat tubes.

(xi) From Section 3.3, it may be noted that only few studies are present which compares the performance of the different fins. From these studies, it can be concluded that out of crimped spiral fins, plain fins, slit fins, and fins with vortex generators (VG), and mixed fins (VGs in front and slits in rear), the slit fins and fins with VGs perform better. However, 
more studies in this direction should be performed to make the results more applicable for the commercial purpose

(xii) In the heat exchangers, a combination of fins can be used and the combinations of the fins can be optimized by analyzing the flow structure and dynamics in the heat exchangers. For that purpose 3D numerical simulations are required, so far, only one has been reported by Tang et al (2009b), which considered a combination of VGs and the slit fins. More combination of this sort can be studied for the practical purpose.

\section{Nomenclature}

$A \quad$ total outside heat transfer surface area $\left(\mathrm{m}^{2}\right)$

$A R \quad$ aspect ratio $(2 \mathrm{~b} / \mathrm{c})$

$A_{c} \quad$ minimum flow area $\left(\mathrm{m}^{2}\right)$

$A_{f r} \quad$ frontal area $\left(\mathrm{m}^{2}\right)$

$a_{1} \quad$ elliptical tube minor axes ( $\left.\mathrm{mm}\right)$

$b_{1} \quad$ elliptical tube major axes $(\mathrm{mm})$

$b \quad$ winglet span $(\mathrm{mm})$

$c \quad$ winglet chord length $(\mathrm{mm})$

$C_{p} \quad$ specific heat $\left(\mathrm{KJ} \mathrm{Kg}^{-1} \mathrm{~K}^{-1}\right)$

$D \quad$ tube outer diameter $(\mathrm{mm})$

$D_{f} \quad$ fin tip diameter (mm)

$D_{h} \quad$ heat exchanger hydraulic diameter $\left(4^{*} A_{c} / P_{w}\right)(\mathrm{mm})$

$e \quad$ tube ellipticity (b1/a1)

$g \quad$ acceleration due to gravity $\left(\mathrm{m} \mathrm{s}^{-2}\right)$

$g_{S} \quad$ segment gap $(\mathrm{mm})$

$f$ friction factor $\left(\frac{2 \Delta P D_{h}}{L \rho U_{f r}^{2}}\right)$ or $\left(\frac{2 \Delta P A_{c} \sigma^{2}}{A \rho U_{f r}^{2}}\right)$

$h \quad$ heat transfer coefficient $\left(\mathrm{W} \mathrm{m}^{-2} \mathrm{~K}^{-1}\right.$ )

$h_{\mathrm{S}} \quad$ height of the segment $(\mathrm{mm})$

$j \quad$ Colburn factor $\left(\frac{N u}{\operatorname{ReP} r^{\frac{1}{3}}}\right)$

$j_{m} \quad$ mass transfer coefficient

$k \quad$ thermal conductivity $\left(\mathrm{W} \mathrm{m}^{-1} \mathrm{~K}^{-1}\right)$

$L \quad$ length of the coil (or array length) $(\mathrm{mm})$

$L_{p f} \quad$ length of the plate fin (mm)

$N_{r} \quad$ number of tube rows

$\triangle P \quad$ pressure drop $(\mathrm{Pa})$

$P_{w} \quad$ wetted perimeter

Pr Prandtl number $\left(\frac{c_{p} \mu}{k}\right)$

$Q \quad$ heat flux (W)

Re Reynolds number based on tube outer diameter and air maximum velocity $\left(\frac{\rho U_{\max } D}{\mu}\right)$

$R e_{a} \quad$ Reynolds number based on array length $\left(\frac{\rho U_{\max } L}{\mu}\right)$

$R e_{c} \quad$ Reynolds number based on tube collar diameter and air maximum velocity $\left(\frac{\rho U_{\max } D_{c}}{\mu}\right)$

$R e_{f r} \quad$ Reynolds number based on tube outer diameter and air frontal velocity $\left(\frac{\rho U_{f r} D_{h}}{\mu}\right)$ 
$R e_{h} \quad$ Reynolds number based on hydraulic diameter $\left(\frac{\rho U_{\max } D_{h}}{\mu}\right)$

$R e_{l w} \quad$ Reynolds number based on the length of the winglet

$R e_{p} \quad$ Reynolds number based on tube perimeter $\left(\frac{\rho U_{\max } D_{h}}{\mu}\right)$ (Jang \& Yang 1998)

$S \quad$ fin spacing $(\mathrm{mm})$

$S_{f} \quad$ fin pitch (mm)

$S_{l} \quad$ longitudinal tube pitch $(\mathrm{mm})$

$S_{t} \quad$ transverse tube pitch $(\mathrm{mm})$

$t_{f} \quad$ fin thickness $(\mathrm{mm})$

$T$ temperature $(\mathrm{K})$

$U \quad$ mean velocity $\left(\mathrm{m} \mathrm{s}^{-1}\right)$

$U_{f r} \quad$ air frontal velocity $\left(\mathrm{m} \mathrm{s}^{-1}\right)$

$U_{\max }$ maximum air velocity in the narrows finned space $\left(\mathrm{m} \mathrm{s}^{-1}\right)$

$W_{p f} \quad$ width of the plate fin (mm)

$W_{s} \quad$ segment width $(\mathrm{mm})$

\section{Greek symbols}

$\alpha \quad$ attack angle

$\beta \quad$ thermal expansion coefficient

$\mu \quad$ dynamic viscosity $\left(\mathrm{Kg} \mathrm{m}^{-1} \mathrm{~s}^{-1}\right)$

$\nu \quad$ kinematic viscosity $\left(\mathrm{m}^{2} \mathrm{~s}^{-1}\right)$

$\varepsilon \quad$ effectiveness

$\in \quad$ turbulent energy dissipation rate $\left(\mathrm{m}^{2} / \mathrm{s}^{3}\right)$

$\eta \quad$ fin efficiency

$\kappa \quad$ turbulent kinetic energy $\left(\mathrm{m}^{2} / \mathrm{s}^{2}\right)$

$\Gamma \quad$ area goodness factor $\left(\frac{Q}{A\left(T_{w}-T_{i}\right)}\right)$ (Kang \& Kim 1999)

$\gamma \quad$ volume goodness factor $\left(\frac{\eta h A}{L A_{f r}}\right)$

$\rho \quad$ density of fluid $\left(\mathrm{kg} \mathrm{m}^{-3}\right)$

$\theta \quad$ wavy angle

$\sigma \quad$ contraction ratio $\left(\frac{A_{f r}}{A_{c}}\right)$

\section{Subscript}

a air

$f \quad$ fin

fr frontal

$I \quad$ inlet

o outlet

$w \quad$ tube wall

\section{References}

Ay H, Jang J Y and Yeh J N 2002 Local heat transfer measurements of plate finned-tube heat exchangers by infrared thermography. Int. J. Heat Mass Transf. 45: 4069-4078

Badr H M 1994 Mixed convection from a straight isothermal tube of elliptic cross section. Int. J. Heat Mass Transf. 37(15): 2343-2365 
Banerjee R K, Karve M, Ha J H and Hwan D 2012 Evaluation of enhanced heat transfer within a four row finned tube array of an air cooled steam condenser. Numer. Heat Transf. Part A: Appl. 61: 735-753

Beecher D T and Fagan T J 1987 Effects of fin pattern on the air-side heat transfer coefficient in plate finned-tube heat exchangers. ASHRAE Trans. 93(2): 1961-1984

Bettanini E 1970 Simulataneuos heat and mass transfer on a vertical surface. Int. Inst. Refrig. Bull. 70(1): 309-317

Bhole M R, Joshi J B and Ramkrishna D 2008 CFD simulation of bubble columns incorporating population balance modelling. Chem. Eng. Sci. 63: 2267-2282

Biswas G, Mitra N K and Fiebig M 1994 Heat transfer enhancement in fin-tube heat exchangers by winglet type vortex generators. Int. J. Heat Mass Transf. 37: 283-291

Brauer H 1964 Compact heat exchangers. Chem. Process Eng. 45(8): 451-460

Briggs D E and Young E H 1963 Convection heat transfer and pressure drop of air flowing across triangular pitch banks of finned tubes. Chem. Eng. Prog. Symp. Ser. 59(41): 1-10

Brockmeier U, Guentermann T and Fiebig M 1993 Performance evaluation of a vortex generator heat transfer surface and comparison with different high performance surfaces. Int. J. Heat Mass Transf. 36: 2575-2587

Chao B and Fagbenle R 1974 On merk's method of calculating boundary layer transfer. Int. J. Heat Mass Transf. 17: 223-240

Chen H T and Hsu W L 2008 Estimation of heat-transfer characteristics on a vertical annular circular fin of finned-tube heat exchangers in forced convection. Int. J. Heat Mass Transf. 51: 1920-1932

Chen H T and Lai J R 2012 Study of heat-transfer characteristics on the fin of two-row plate finned-tube heat exchangers. Int. J. Heat Mass Transf. 55: 4088-4095

Chen Y, Fiebig M and Mitra N 1998a Conjugate heat transfer of a finned oval tube with a punched longitudinal vortex generator in form of a delta winglet: parametric investigations of the winglet. Int. J. Heat Mass Transf. 41: 3961-3978

Chen Y, Fiebig M and Mitra N 1998b Heat transfer enhancement of a finned oval tube with punched longitudinal vortex generators inline. Int. J. Heat Mass Transf. 41: 3040-3055

Chen Y, Fiebig M and Mitra N 2000 Heat transfer enhancement of $®$ nned oval tubes with staggered punched longitudinal vortex generators. Int. J. Heat Mass Transf. 43: 417-435

Chen H T, Song J P and Wang Y T 2005 Prediction of heat transfer coefficient on the fin inside one-tube plate finned-tube heat exchangers. Int. J. Heat Mass Transf. 48: 2697-2707

Chen H T, Chou J C and Wang H C 2007 Estimation of heat transfer coefficient on the vertical plate fin of finned-tube heat exchangers for various air speeds and fin spacings. Int. J. Heat Mass Transf. 50: 45-57

Cheng Y P, Qu Z G, Tao W Q and He Y L 2004 Numerical design of efficient slotted fin surface based on the field synergy principle. Numer. Heat Transf. Part A: Appl. 45: 517-538

Cheng Y P, Lee T S and Low H T 2007 Numerical analysis of periodically developed fluid flow and heat transfer characteristics in the triangular wavy fin-and-tube heat exchanger based on field synergy principle. Numer. Heat Transf. Part A: Appl. 53: 821-842

Cheng Y P, Lee T S and Low H T 2009 Numerical prediction of periodically developed fluid flow and heat transfer characteristics in the sinusoid wavy fin-and-tube heat exchanger. Int. J. Numer. Methods Heat Fluid Flow 19: 728-74

Choi J M, Kim Y, Lee M and Kim Y 2010 Air side heat transfer coefficients of discrete plate finned-tube heat exchangers with large fin pitch. Appl. Therm. Eng. 30: 174-180

Chokeman Y and Wongwises S 2005 Effect of fin pattern on the air side performance of herringbone wavy fin-and-tube heat exchangers. Heat Mass Transf. 41: 642-650

Colburn A P 1942 Heat transfer by natural and forced convection. Eng. Bull. Purdue Univ. Res. Ser. 26(84): $47-50$

Crow S C 1970 Stability theory for a pair of trailing vortices. AIAA J. 8: 2172-2179

Dejong N C and Jacobi A M 1997 An experimental study of flow and heat transfer in parallel-plate arrays: local, row-by-row and surface average behavior. Int. J. Heat mass Transf. 40: 1365-1378

Du Y J and Wang C C 2000 An experimental study of the airside performance of the superslit fin-and-tube heat exchangers. Int. J. Heat Mass Transf. 43: 4475-4482 
Eckels P W and Rabas T J 1987 Dehumidification: on the correlation of wet and dry transport processes in plate finned-tube heat exchangers. J. Heat Transf. 109: 575-582

Ekambara K, Dhotre M T and Joshi J B 2005 CFD simulations of bubble column reactors: 1D, 2D and 3D appraoch. Chem. Eng. Sci. 60: 6733-6746

Elmahdy A H 1975 Analytical and experimental multi-row, finned-tube heat exchanger performance during cooling and dehumidification process. Ph.D. thesis, Mech. Eng. Dept., Carleton Univ. Ottawa, Canada

Elmahdy A H and Biggs R C 1979 Finned tube heat exchanger: correlation of dry surface heat transfer data. ASHRAE Trans. 85(2): 262-273

ElSherbini A I and Jacobi A M 2002 The thermal-hydraulic impact of delta-wing vortex generators on the performance of a plain-fin-and-tube heat exchanger. HVAC\&R Res. 8(4): 357-370

Erek A, Özerdem B, Bilir L and İlken Z 2005 Effect of geometrical parameters on heat transfer and pressure drop characteristics of plate fin and tube heat exchangers. Appl. Therm. Eng. 25: 2421-2431

Fiebig M 1998 Vortices, generators and heat transfer. Inst. Chem. Engrs. Trans. IChemE 76: 108-122

Fiebig M, Valencia A and Mitra N K 1993 Wing-type vortex generators for fin-and-tube heat exchangers. Exp. Therm. Fluid Sci. 7: 287-295

Fiebig M, Valencia A and Mitra N K 1994 Local heat transfer and flow losses in fin-and-tube heat exchangers with vortex generators: a comparison of round and flat tubes. Exp. Therm. Fluid Sci. 8: 35-45

Fiebig M, Chen Y, Grosse-Gorgemann A and Mitra N K 1995 Numerical analysis of heat transfer and flow loss in a parallel plate heat exchanger element with longitudinal vortex generators as fins. J. Heat Transf. Trans. ASME 117: 1064-1067

Ganapathy V 2003 Industrial boilers and heat recovery steam generators: design, applications and calculations. Marcel Dekker

Gray D L and Webb R L 1986 Heat transfer and friction correlations for plate fin-and-tube heat exchangers having plain fins, in: Proceedings of the Eighth International Heat Transfer Conference, San Francisco, California 6: 2745-2750

Guillory J and McQuiston F 1973 An experimental investigation of air dehumidification in a parallel plate. ASHRAE Trans. 79(2): 146-151

Hashizume K, Morikawa R, Koyama T and Matsue T 2002 Fin efficiency of serrated fins. Heat Transf. Eng. 23: 6-14

He Y L, Tao W Q, Song F Q and Zhang W 2005 Three-dimensional numerical study of heat transfer characteristics of plain plate fin-and-tube heat exchangers from view point of field synergy principle. Int. J. Heat Fluid Flow 26: 459-473

Hu X and Jacobi A M 1993 Local heat transfer behavior and its impact on a single-row annularly finned tube heat exchanger. Trans. ASME 115: 66-74

Huang C H, Yuan I C and Ay H 2003 A three-dimensional inverse problem in imaging the local heat transfer coefficients for plate finned-tube heat exchangers. Int. J. Heat Mass Transf. 46: 3629-3638

Huang C H, Yuan I C and Ay H 2009 An experimental study in determining the local heat transfer coefficients for the plate finned-tube heat exchangers. Int. J. Heat Mass Transf. 52: 4883-4893

Ibrahim T A and Gomaa A 2009 Thermal performance criteria of elliptic tube bundle in crossflow. Int. J. Therm. Sci. 48: 2148-2158

Idem S A, Jacobi A M and Goldchrnidt V M 1990 Heat transfer characterization of a finned-tube heat exchanger (with and without condensation). Trans. ASME 112: 64-70

Idem S A and Goldchmidt V M 1993 Sensible and latent heat transfer to a baffled finned-tube heat exchanger. Heat Transf. Eng. 14(3): 26-35

Ishiguro H, Nagata S, Yabe A and Nariai H 1991 Augmentation of forced-convection heat transfer by applying electric fields to disturb flow near a wall. ASME J. 3: 25-31

Jacobi A M and Goldschmidt V W 1990 Low Reynolds number heat and mass transfer measurements of an overall counterflow, baffled, finned-tube, condensing heat exchanger. Int. J. Heat Mass Transf. 33(4): $755-765$

Jacobi A M and Shah R K 1995 Heat transfer surface enhancement through the use of longitudinal vortices: A review of recent progress. Exp. Therm. Fluid Sci. 11: 295-30 
Jakob M 1938 Heat transfer and flow resistance in cross flow of gases over tube banks. Trans. ASME 60: 384-386

Jang J Y and Yang J Y 1998 Experimental and 3d numerical analysis of the thermal-hydraulic characteristics of elliptic finned-tube heat exchangers. Heat Transf. Eng. 19(4): 55-67

Jin W W, He Y L, Qu Z G, Zhang C C and Tao W Q 2006 Optimum design of two-row slotted fin surface with $\mathrm{X}$-shape strip arrangement positioned by "front coarse and rear dense" principle part ii: results and discussion. Numer. Heat Transf. Part A Appl. 50: 751-771

Joardar A and Jacobi A M 2008 Heat transfer enhancement by winglet-type vortex generator arrays in compact plain-fin-and-tube heat exchangers. Int. J. Refrig. 31: 87-9

Joshi J B and Ranade V V 2003 Computational fluid dynamics for desiging process eqiupment expectations, current status and path forward. Ind. Eng. Chem. Res. 42: 1115-1128

Joshi J B and Shah Y T 1981 Gas-liquid solid reactor design, in: Mah, R S H and Sieder, W D (Eds.) Proceedings of the Engineering Foundation Conference. AIChE J. 277-333

Joshi J B and Sharma M M 1976 Mass transfer characteristics of horizontal sparged contactors. Trans. Inst. Chem. Eng. UK 54: 42-53

Joshi J B and Sharma M M 1977 Mass transfer and hydrodynamic characteristics of gas inducing type of agitated contactors. Can. J. Chem. Eng. 55: 683-695

Joshi J B and Sharma M M 1978 Liquid phase backmixing in sparged contactors. Can. J. Chem. Eng. 56: $116-119$

Joshi J B, Tabib M V, Deshpande S S and Mathpati C S 2009 Dynamics of flow structures and transport phenomena-1: Experimental and numerical techniques for identification and energy content of flow structures. Ind. Eng. Chem. Res. 48: 8244-8284

Joshi J B, Nere N K, Rane C V, Murthy B N, Mathpati C S, Patwardhan A W and Ranade V V 2011a CFD simulations of stirred tanks: comparison of turbulence models, Part I: radial flow impellers. Can. J. Chem. Eng. 89: 23-82

Joshi J B, Nere N K, Rane C V, Murthy B N, Mathpati C S, Patwardhan A W and Ranade V V 2011b CFD simulations of stirred tanks: comparison of turbulence models, Part II: axial flow impellers, multiple impellers and multiphase dispersions. Can. J. Chem. Eng. 89: 754-816

Kang H C and Kim M H 1999 Effect of strip location on the air-side pressure drop and heat transfer in strip fin-and-tube heat exchanger. Int. J. Refrig. 22: 302-312

Kang H J, Li W, Li H J, Xin R C and Tao W Q 1994 Experimental study on heat transfer and pressure drop characteristics of four types of plate fin-and-tube heat exchanger surfaces. J. Therm. Sci. 3: 34-42

Kawaguchi K, Okui K and Kashi T 2004 The heat transfer and pressure drop characteristics of finned tube banks in forced convection (comparison of the pressure drop characteristics of spiral fins and serrated fins). Heat Transf. Res. 33: 431-444

Kayansayan N 1993 Heat transfer characterization of flat plain fins and round tube heat exchangers. Exp. Therm. Fluid Sci. 6: 263-272

Kays W M and London A L 1950 Heat-transfer and flow-friction characteristics of some compact heatexchanger surfaces. Trans. ASME 72: 1087-1097

Kays W M and London A L 1955 Compact heat exchangers, second edition, McGraw Hill 7-224

Kim N H, Youn B and Webb R L 1999 Air-side heat transfer and friction correlation for plain fin and tube heat exchangers with staggered tube arrangements. J. Heat Transfer. 121: 662-667

Kreith F and Bohn M S 1993 Principles of heat transfer, fifth ed., New York: West Publishing Co

Kulacki F A 1983 In: Augmentation of low reynolds number forced convection channel flow by electrostatic discharge, in low Reynolds number flow heat exchangers, S Kakac (ed.), Washington, Hemisphere, 753-782

Kulkarni A A, Joshi J B, Ravikumar V and Kulkarni B D 2001 Application of multi-resolution analysis for simultaneous measurement of gas and liquid velocities and fractional gas hold-up in bubble column using LDA. Chem. Eng. Sci. 56: 5037-5048

Kumaresan T and Joshi J B 2006 Effect of impeller design on the flow pattern and mixing in stirred tanks. Chem. Eng. J. 115: 173-193 
Kuvannarat T, Wang C C and Wongwises S 2006 Effect of fin thickness on the air-side performance of wavy fin-and-tube heat exchangers under dehumidifying conditions. Int. J. Heat Mass Transf. 49: 25872596

Kwak K, Torii K and Nishino K 2002 Heat transfer and flow characteristics of fin-tube bundles with and without winglet-type vortex generators. Exp. Fluids 33: 696-702

Kwak K, Torii K and Nishino K 2003 Heat transfer and pressure loss penalty for the number of tube rows of staggered finned-tube bundles with a single transverse row of winglets. Int. J. Heat Mass Transf. 43: $417-435$

Kwak K M, Torii K and Nishino K 2005 Simultaneous heat transfer enhancement and pressure loss reduction for finned-tube bundles with the first or two transverse rows of built-in winglets. Exp. Therm. Fluid Sci. 29: 625-632

Lemouedda A, Schmid A, Franz E, Breuer M and Delgad A 2011 Numerical investigations for the optimization of serrated finned-tube heat exchangers. Appl. Therm. Eng. 31: 1393-1401

Lin C W and Jang J Y 2005 3D Numerical heat transfer and fluid flow analysis in plate-fin and tube heat exchangers with electrohydrodynamic enhancement. Heat Mass Transf. 41: 583-593

Liu Y C, Wongwises S, Chang W J and Wang C C 2010 Airside performance of fin-and-tube heat exchangers in dehumidifying conditions - Data with larger diameter. Int. J. Heat Mass Transf. 53: 16031608

London A L and Ferguson C K 1949 Test results of high-performance heat-exchanger surfaces used in aircraft intercoolers and their significance for gas-turbine regenerator design. Trans. ASME 71: 17-26

Ma Y, Yuan Y, Liu Y, Hu X and Huang Y 2012 Experimental investigation of heat transfer and pressure drop in serrated finned tube banks with staggered layouts. Appl. Therm. Eng. 37: 314-323

Madi M A, Johns R A and Heikal M R 1998 Performance characteristics correlation for round tube and plate finned heat exchangers. Int. J. Refrig. 21(7): 507-517

Martinez E, Vicente W, Soto G and Salinas M 2010 Comparative analysis of heat transfer and pressure drop in helically segmented finned tube heat exchangers. Appl. Therm. Eng. 30: 1470-1476

Mathpati C S, Tabib M V, Deshpande S S and Joshi J B 2009 Dynamics of flow structures and transport phenomena-2: Relationship with design objectives and design optimization. Ind. Eng. Chem. Res. 48: $8285-8311$

Matos R S, Vargas J V C, Laursen T A and Saboya F E M 2001 Optimization study and heat transfer comparison of staggered circular and elliptical tubes in forced convection. Int. J. Therm. Sci. 44: 39533961

Matos R S, Laursen T A, Vargas J V C and Bejan A 2004a Three-dimensional optimization of staggered finned circular and elliptic tubes in forced convection. Int. J. Therm. Sci. 43: 477-487

Matos R S, Vargas J V C, Laursen T A and Bejan A 2004b Optimally staggered finned circular and elliptic tubes in forced convection. Int. J. Heat Mass Transf. 47: 1347-1359

McQuiston F C 1978a Heat, mass and momentum transfer data for five plate-fin-tube heat trasnfer surfaces. ASHRAE Trans. 84(1): 266-293

McQuiston F C 1978b Correlation of heat, mass and momentum transport coefficient for plate-fin-tube heat trasnfer surfaces with staggered tubes. ASHRAE Trans. 84(1): 294-308

McQuiston F C 1981 Finned tube heat exchangers: state of the art for the air side. ASHRAE Trans. 87: 1077-1085

Merkin J 1977 Free Convection boundary layers on cylinders of elliptic cross section. ASME J. Heat Transf. 99: 453-457

Mirth D R and Ramadhyani S 1993 Prediction of cooling-coil performance under condensing conditions. Int. J. Heat Fluid Flow 14(4): 391-400

Mirth D R and Ramadhyani S 1994 Correlations for predicting the air-side Nusselt numbers and friction factors in chilled-water cooling coils. Exp. Heat Transf. 7: 143-162

Mochizuki S, Yagi Y and Yang W J 1987 Transport phenomena in stacks of interrupted parallel-plate surfaces. Exp. Heat Transf. 1: 127-140

Mon M S and Gross U 2004 Numerical study of fin-spacing effects in annular-finned tube heat exchangers. Int. J. Heat Mass Transf. 47: 1953-1964 
Mullisen R S and Loehrke R I 1986 A study of the flow mechanism responsible for the heat transfer enhancement in interrupted-plate heat exchangers. ASME J. Heat Tranf. 108: 377-385

Murthy B N and Joshi J B 2008 Assesment of standard $\mathrm{k}-\epsilon$, rsm and les turbulence models in a baffled stirred vessel agitated by various impeller designs. Chem. Eng. Sci. 63: 5468-5495

Murthy B N, Deshmukh N, Patwardhan A W and Joshi J B 2007a Hollow self-inducing impeller: flow visualisation and CFD simulation. Chem. Eng. Sci. 62: 3839-3848

Murthy B N, Ghadge R S and Joshi J B 2007b CFD simulations of gas-liquid-solid stirred reactor: prediction of critical impeller speed for solid suspension. Chem. Eng. Sci. 62: 7184-7195

Myers R J 1967 The effect of dehumidification on the air-side heat transfer coefficient for finned-tube coil. MS Thesis, Mech. Eng. Dept., Univ. Minnesota, Minneapolis, MN

Næss E 2010 Experimental investigation of heat transfer and pressure drop in serrated-fin tube bundles with staggered tube layouts. Appl. Therm. Eng. 30: 1531-1537

Nakayama W and Xu L P 1983 Enhanced fins for air-cooled heat exchangers - heat transfer and friction correlations, in: Proc. 1st ASME/JSME Therm. Eng. Joint Conf. 1: 495-502

Naphon P and Wongwises S 2005 Heat transfer coefficients under dry- and wet-surface conditions for a spirally coiled finned tube heat exchanger. Int. Commun. Heat Mass Transf. 32: 371-385

Nelson D A, Ohadi M M, Zia S and Whipple R L 1991 Electrostatic effects on heat transfer and pressure drop in cylindrical geometries. ASME J. 3: 33-39

Nere N K, Patwardhan A W and Joshi J B 2003 Liquid phase mixing in stirred vessels: turbulent flow regime. Ind. Eng. Chem. Res. 42: 2661-2698

Nir A 1991 Heat transfer and friction factor correlations for crossflow over staggered finned tube banks. Heat Transf. Eng. 12: 43-58

Nuntaphan A, Kiatsiriroat T and Wang C C 2005a Air side performance at low Reynolds number of crossflow heat exchanger using crimped spiral fins. Int. Commun. Heat Mass Transf. 32: 151-165

Nuntaphan A, Kiatsiriroat T and Wang C C 2005b Heat transfer and friction characteristics of crimped spiral finned heat exchangers with dehumidification. Appl. Therm. Eng. 25: 327-340

Ogata J, Iwafuji Y, Shimada Y and Yamazaki T 1992 Boiling heat transfer enhancement in tube-bundle evaporators utilizing electric field effects. ASHRAE Trans. 98(2): 435-444

Ohadi M M, Nelson D A and Zia S 1991 Heat transfer enhancement of laminar and turbulent pipe flow via corona discharge. Heat Mass Transf. J. 4: 1175-1187

Ota T, Nishiyama H and Taoka Y 1984 Heat transfer and flow around and elliptic cylinder. Int. J. Heat Mass Transf. 27: 1771-1779

Paeng J G, Kim K H and Yoon Y H 2010 Experimental measurement and numerical computation of the air side convective heat transfer coefficients in a plate fin-tube heat exchanger. J. Mech. Sci. Technol. 23: 536-543

Patwardhan A W and Joshi J B 1999 Relation between flow pattern and blending in stirred tanks. Ind. Eng. Chem. Res. 38: 3131-3143

Pesteei S M, Subbarao P M V and Agarwal R S 2005 Experimental study of the effect of winglet location on heat transfer enhancement and pressure drop in fin-tube heat exchangers. Appl. Therm. Eng. 25: 16841696

Pirompugd W, Wongwises S and Wang C C 2005 A tube-by-tube reduction method for simultaneous heat and mass transfer characteristics for plain fin-and-tube heat exchangers in dehumidifying conditions. Heat Mass Transf. 41: 756-765

Pirompugd W, Wongwises S and Wang C C 2006 Simultaneous heat and mass transfer characteristics for wavy fin-and-tube heat exchangers under dehumidifying conditions. Int. J. Heat Mass Transf. 49: 132143

Pirompugd W, Wang C C and Wongwises S 2007a Finite circular fin method for heat and mass transfer characteristics for plain fin-and-tube heat exchangers under fully and partially wet surface conditions. Int. J. Heat Mass Transf. 50: 552-556

Pirompugd W, Wang C C and Wongwises S 2007b A fully wet and fully dry tiny circular fin method for heat and mass transfer characteristics for plain fin-and- tube heat exchangers under dehumidifying conditions. J. Heat Transf. 129(9): 1256-1267 
Pirompugd W, Wang C C and Wongwises S 2008 Finite circular fin method for wavy fin-and-tube heat exchangers under fully and partially wet surface conditions. Int. J. Heat Mass Transf. 51: 4002-4017

Pirompugd W, Wang C C and Wongwises S 2009 A review on reduction method for heat and mass transfer characteristics of fin-and-tube heat exchangers under dehumidifying conditions. Int. J. Heat Mass Transf. 52: 2370-2378

Pongsoi P, Pikulkajorn S, Wang C C and Wongwises S 2011 Effect of fin pitches on the air-side performance of crimped spiral fin-and-tube heat exchangers with a multipass parallel and counter cross-flow configuration. Int. J. Heat Mass Transf. 54: 2234-2240

Pongsoi P, Pikulkajorn S and Wongwises S 2012a Experimental study on the air-side performance of a multipass parallel and counter cross-flow L-footed spiral fin-and-tube heat exchanger. Heat Transf. Eng. 33: $1251-1263$

Pongsoi P, Pikulkajorn S and Wongwises S 2012b Effect of fin pitches on the optimum heat transfer performance of crimped spiral fin-and-tube heat exchangers. Int. J. Heat Mass Transf. 55: 6555-6566

Pongsoi P, Pikulkajorn S, Wang C C and Wongwises S 2012c Effect of number of tube rows on the airside performance of crimped spiral fin-and-tube heat exchanger with a multipass parallel and counter cross-flow configuration. Int. J. Heat Mass Transf. 55: 1403-1411

Pongsoi P, Promoppatum P, Pikulkajorn S and Wongwises S 2013 Effect of fin pitches on the air-side performance of L-footed spiral fin-and-tube heat exchangers. Int. J. Heat Mass Transf. 59: 75-82

Poulter R and Allen PHG 1986 Electrohydrodynamically augmented heat and mass transfer in the shell/tube heat exchanger, in: Proceedings of the 8th international heat transfer conference, San Francisco, 29632968

Qu Z G, Tao W Q and He Y L 2004 Three-dimensional numerical simulation on laminar heat transfer and fluid flow characteristics of strip fin surface with x-arrangement of strips. J. Heat Transf. 126: 697-707

Raghav Rao K S M S, Rewatkar V B and Joshi J B 1988 Critical impeller speed for solid suspension in mechanically agitated solid liquid contactors. A. I. Ch. E. J. 34: 1332-1340

Raithby G D and Hollands K G T 1985 Natural convection, in: Handbook of heat transfer fundamentals, second ed., W M Rohsenow, J P Hartnett and E N Ganic (eds), New York, McGraw-Hill

Ranade V V and Joshi J B 1990 Flow generated by a disc turbine I: experimental. Trans. Inst Chem. Eng. (UK)-A: Chem. Eng. Res. Des. 68: 19-33

Ranade V V, Joshi J B and Marathe A G 1989 Flow generated by pitched blade turbine part II: mathematical modelling and comparison with the experimental data. Chem. Eng. Commun. 81: 225-248

Ranade V V, Mishra V P, Saraph V S, Deshpande G B and Joshi J B 1992 Comparison of axial flow impellers using LDA. Ind. Eng. Chem. Res. 31: 2370-2379

Rewatkar V B and Joshi J B 1991 Critical impeller speed for solid suspension in mechanically agitated three phase reactors I: experimental part. Ind. Eng. Chem. Res. 30: 1770-1784

Rich D G 1973 The effect of fin spacing on the itcat transfer and friction performance of multi-row, smooth plate fin-and-tube heat exchangers. ASHRAE Trans. 79(2): 137-145

Rich D G 1975 The Effect of the number of tube rows on heat transfer performance of smooth plate fin-and-tube heat exchangers. ASHRAE Trans. 81(1): 307-317

Robinson K K and Briggs D E 1966 Pressure drop of air flowing across triangular pitch banks of finned tubes. Chem. Eng. Prog. Symp. Ser. 62(64): 177-184

Rocha L A O, Saboya F E M and Vargas J V C 1997 A comparative study of elliptical and circular sections in one- and two-row tubes and plate fin heat exchangers. Int. J. Heat Fluid Flow 18: 247-252

Romero-Mendez R, Sen M, Yang K T and McClain R L 1997 Effect of tube-to-tube conduction on plate-fin and tube heat exchanger performance. Int. J. Heat Mass Transf. 40(16): 3909-3916

Rosman E C, Carajilescov P and Saboya F E M 1984 Performance of one and two-row tube and plate fin heat exchangers. ASME J. Heat Transf. 106: 627-632

Saboya S M and Saboya F E M 2001 Experiments on elliptic sections in one- and two-row arrangements of plate fin and tube heat exchangers. Exp. Therm. Fluid Sci. 24: 67-75

Saboya F E M and Sparrow E M 1976 Transfer characteristics of two-row plate fin and tube heat exchangers configurations. Int. J. Heat Mass Transf. 19: 41-49 
Sahiti N, Durst F and Dewan A 2006 Strategy for selection of elements for heat transfer enhancement. Int. J. Heat Mass Transf. 49: 3392-3400

Saechan P and Wongwises S 2008 Optimal configuration of cross flow plate finned tube condenser based on the second law of thermodynamics. Int. J. Therm. Sci. 47: 1473-1481

Seshimo Y and Fujii M 1991 An experimentak study of the performance of plate fin and tube heat exchangers at low Reynolds number. 3rd ASME/JSME Therm. Eng. Jt. Conf. 4: 449-454

Sheu T W H and Tsai S 1999 A comparison study on fin surfaces in finned-tube heat exchangers. Int. J. Numer. Methods Heat Fluid Flow 9: 92-106

Taguchi G 1991 Taguchi on robust technology development. Bring quality engineering (QE) Upstream. ASME

Taguchi G, Elsayed A E and Thomas C H 1989 Quality engineering in production systems. New York: McGraw-Hill

Tang L H, Xie G N, Zeng M, Wang H G, Yan X H and Wang Q W 2007a Experimental investigation on heat transfer and flow friction characteristics in three types of plate fin-and-tube heat exchangers. J. Xi'an Jiaotong Univ. 41: 521-525

Tang L H, Xie G N, Zeng M and Wang Q W 2007b Numerical simulation of fin patterns on air-side heat transfer and flow friction characteristics of fin-and-tube heat exchangers, in: Proceedings of ASCHT07, First Asian Symp. Comp. Heat Transf. Fluid Flow, Xi'an, China

Tang L H, Min Z, Xie G N and Wang Q W 2009a Fin pattern effects on air-side heat transfer and friction characteristics of fin-and-tube heat exchangers with large number of large-diameter tube rows. Heat Transf. Eng. 30: 171-180

Tang L H, Zeng M and Wang Q W 2009b Experimental and numerical investigation on air-side performance of fin-and-tube heat exchangers with various fin patterns. Exp. Therm. Fluid Sci. 33: 818-827

Tao W Q, Jin W W, He Y L, Qu Z G and Zhang C C 2006 Optimum design of two-row slotted fin surface with $\mathrm{x}$-shape strip arrangement positioned by "front coarse and rear dense"principle, part I: physical/mathematical models and numerical methods. Numer. Heat Transf. Part A 50: 731-749

Tao Y B, He Y L, Huang J, Wu Z G and Tao W Q 2007a Numerical study of local heat transfer coefficient and fin efficiency of wavy fin-and-tube heat exchangers. Int. J. Therm. Sci. 46: 768-778

Tao W Q, Cheng Y P and Lee T S 2007b The influence of strip location on the pressure drop and heat transfer performance of a slotted fin. Numer. Heat Transf. Part A Appl. 52: 463-480

Tao Y, He Y, Qu Z and Tao W 2011 Numerical study on performance and fin efficiency of wavy fin-andtube heat exchangers. Prog. Comput. Fluid Dyn. 11: 246-254

Thakre S S and Joshi J B 1999 CFD simulation of flow in bubble column reactors: importance of drag force formulations. Chem. Eng. Sci. 54: 5055-5060

Thorat B N, Shevade A V, Bhilegaonkar K R, Agalave R H, Parasu Veera U, Thakre S S, Pandit A B, Sawant S B and Joshi J B 1998 Effect of sparger design and height to diameter ratio on gas hold-up in bubble column reactors. Trans. Instn. Chem. Engrs. - A: Chem. Eng. Res. Des. 76: 823-834

Threlkeld J L 1970 Thermal environmental engineering. New York: Prentice-Hall

Tian L, He Y, Tao Y and Tao W 2009 A comparative study on the air-side performance of wavy fin-andtube heat exchanger with punched delta winglets in staggered and in-line arrangements. Int. J. Therm. Sci. 48: 1765-1776

Tiggelbeck St., Mitra N K and Fiebig M 1994 Comparison of wing-type vortex generators for heat transfer enhancement for heat transfer enhancement in channel flows. Trans. ASME 116: 880-885

Torii K, Kwak K M and Nishino K 2002 Heat transfer enhancement accompanying pressure-loss reduction with winglet-type vortex generators for fin-tube heat exchangers. Int. J. Heat Mass Transf. 45: 3795-3801

Tree D and Helmer W 1976 Experimental heat and mass transfer data for condensing flow in a parallel plate heat exchanger. ASHRAE Trans. 82: 289-299

Tsai S F and Sheu W H 1998 Some physical insights into a two-row finned-tube heat transfer

Tsai S F, Sheu T W H and Lee S M 1998 Heat transfer in a conjugate heat exchanger with a wavy fin surface. Int. J. Heat Mass Transf. 42: 1735-1745

Wang C C, Chang Y J, Hsieh Y C and Lin Y T 1996 Sensible heat and friction characteristics of plate fin-and-tube heat exchangers having plane fins. Int. J. Refrig. 19(4): 223-230 
Wang C C and Chi K Y 2000 Heat transfer and friction characteristics of plain fin-and-tube heat exchangers part I: new experimental data. Int. J. Heat Mass Transf. 43: 2681-2691

Wang J and Hihara E 2003 Prediction of air coil performance under partially wet and totally wet cooling conditions using equivalent dry-bulb temperature method. Int. J. Refrig. 26: 293-301

Wang C C, Fu W L and Chang C T 1997 Heat transfer and friction characteristics of typical wavy fin-andtube heat exchangers. Exp. Therm. Fluid Sci. 14: 174-186

Wang C C, Tao W H and Chang C J 1999 An investigation of the airside performance of the slit fin-and-tube heat exchangers. Int. J. Refrig. 22: 595-603

Wang C C, Chi K Y and Chang C J 2000 Heat transfer and friction characteristics of plain fin-and-tube heat exchangers part II: Correlation. Int. J. Heat Mass Transf. 43: 2693-2700

Wang C C, Tao W H and Du Y J 2000b Effect of waffle height on the air-side performance of wavy fin-and-tube heat exchangers under dehumidifying conditions. Heat Trasnf. Eng. 21: 17-26

Wang C C, Webb R L and Chi K Y 2000a Data reduction for air-side performance of fin-and-tube heat exchangers. Int. J. Heat Mass Transf. 43: 2693-2700

Wang C C, Hwang Y M and Lin Y T 2002 Empirical correlations for heat transfer and flow friction characteristics of herringbone wavy fin-and-tube heat exchangers. Int. J. Refrig. 25(5): 673-680

Wangnippanto S, Tiansuwan J, Jiracheewanun S, Wang C C and Kiatsiriroat T 2001 Air side performance of thermosyphon heat exchanger in low Reynolds number region with and without electric field. Energy Conserv. Manag. 43: 1791-1800

Watel B, Harmand S and Desmet B 1999 Influence of flow velocity and fin sapcing on the forced convective heat trasnfer from an annular-finned tube. JSME Int. J. 42(1): 56-64

Watel B, Harmand S and Desmet B 2000a Influence of fin spacing and rotational speed on the convective heat exchanges from a rotating finned tube. Int. J. Heat Fluid Flow 21(2): 221-227

Watel B, Harmand S and Desmet B 2000b Experimental study of convective heat transfer from a rotating finned tube in transverse air flow. Exp. Fluids 29: 79-90

Webb R L 1990 Air-side heat transfer correlations for flat and wavy plate fin-and-tube geometries. ASHRAE Trans. 96(2): 445-449

Webb P L 1994 Principles of enhanced heal transfer. New York: Wiley

Weierman C 1976 Correlations ease the selection of finned tubes. Oil Gas J. 74(36): 94-100

Weierman C, Taborek J and Marner W J 1978 Comparison of the performance of in-line and staggered banks of tubes with segmented fins. The American Inst. Chem. Engs. Symp. 74(174): 39-46

Wongwises S and Chokeman Y 2004 Effect of fin thickness on air-side performance of herringbone wavy fin-and-tube heat exchangers. Heat Mass Transf. 41: 147-154

Wongwises S and Chokeman Y 2005 Effect of fin pitch and number of tube rows on the air side performance of herringbone wavy fin and tube heat exchangers. Energy Convers. Manag. 46: 2216-2231

Wongwises S and Naphon P 2006a Thermal performance of a spirally coiled finned tube heat exchanger under wet-surface conditions. Heat Transf. Eng. 20(2): 212-226

Wongwises S and Naphon P 2006b Heat transfer characteristics of a spirally coiled, finned-tube heat exchanger under dry-surface conditions. Heat Transf. Eng. 27: 25-34

Xie G, Wang Q and Sunden B 2009 Parametric study and multiple correlations on air-side heat transfer and friction characteristics of fin-and-tube heat exchangers with large number of large-diameter tube rows. Appl. Therm. Eng. 29: 1-16

Xin R C, Li H Z, Kang H J, Li W and Tao W Q 1994 An experimental investigation on heat transfer and pressure drop characteristics of triangular wavy fin-and-tube heat exchanger surfaces. J. Xi'an Jiaotong Univ. 28(2): 77-83

XueMei H, Tao L, Yan G and ShiQian X 2011 Numerical study of the serpentine finned tube used in air cooled condenser. IEEE

Yabe A 1991 Active heat transfer enhancement by applying electric fields. ASME J. 3: 15-23

Yabe A, Mori Y and Hijikata K 1978 EHD study of the corona wind between wire and plate electrode. AIAA J. 16(4): 340-345

Yabe A, Mori Y and Hijikata K 1987 Heat transfer enhancement techniques utilizing electric fields. Heat Transfer High Technol. Power Engineer 394-405 
Yan W M and Sheen P J 2000 Heat transfer and friction characteristics of fin-and-tube heat exchangers. Int. J. Heat Mass Transf. 43: 1651-1659

Yang L, Tan H, Du X and Yang Y 2012 Thermal-flow characteristics of the new wave-finned flat tube bundles in air-cooled condensers. Int. J. Therm. Sci. 53: 166-174

Yoshii T, Yamamoto M and Otaki T 1973 Effects of dropwise condensate on wet surface heat transfer of air cooling coils. Proc. $13^{\text {th }}$ Int. Congress Refrig. 285-292

Yun J Y and Lee K S 1999 Investigation of heat transfer characteristics on various kinds of fin-and-tube heat exchangers with interrupted surfaces. Int. J. Heat Mass Transf. 42(13): 2375-2385

Yun J and Lee K 2000 Influence of design parameters on the heat transfer and flow friction characteristics of the heat exchanger with slit fins. Int. J. Heat Mass Transf. 43: 2529-2539

Zeng M, Tang L H, Lin M and Wang Q W 2010 Optimization of heat exchangers with vortex-generator fin by Taguchi method. Appl. Therm. Eng. 30: 1775-1783

Zhang L W, Balachandar S, Tafti D K and Najjar F M 1997 Heat transfer enhancement mechanisms in inline and staggered parallel-plate fin heat exchangers. Int. J. Heat Mass Transf. 40: 2307-2325

ZukausKas A A 1972 Heat transfer from tubes in cross flow. Adv. Heat Transf. 8: 93-160 